\title{
An mHealth intervention for the dietary management of hemodialysis patients
}

Citation for published version (APA):

Fakih El Khoury, C. (2020). An mHealth intervention for the dietary management of hemodialysis patients. [Doctoral Thesis, Maastricht University]. Ridderprint BV. https://doi.org/10.26481/dis.20200701cf

Document status and date:

Published: 01/01/2020

DOI:

$10.26481 /$ dis.20200701cf

Document Version:

Publisher's PDF, also known as Version of record

\section{Please check the document version of this publication:}

- A submitted manuscript is the version of the article upon submission and before peer-review. There can be important differences between the submitted version and the official published version of record.

People interested in the research are advised to contact the author for the final version of the publication, or visit the DOI to the publisher's website.

- The final author version and the galley proof are versions of the publication after peer review.

- The final published version features the final layout of the paper including the volume, issue and page numbers.

Link to publication

\footnotetext{
General rights rights.

- You may freely distribute the URL identifying the publication in the public portal. please follow below link for the End User Agreement:

www.umlib.nl/taverne-license

Take down policy

If you believe that this document breaches copyright please contact us at:

repository@maastrichtuniversity.nl

providing details and we will investigate your claim.
}

Copyright and moral rights for the publications made accessible in the public portal are retained by the authors and/or other copyright owners and it is a condition of accessing publications that users recognise and abide by the legal requirements associated with these

- Users may download and print one copy of any publication from the public portal for the purpose of private study or research.

- You may not further distribute the material or use it for any profit-making activity or commercial gain

If the publication is distributed under the terms of Article $25 \mathrm{fa}$ of the Dutch Copyright Act, indicated by the "Taverne" license above, 


\section{An mHealth intervention for the dietary management of hemodialysis patients}


The research presented in this thesis was conducted at Care and Public Health Research Institute (CAPHRI), Department of Health Services Research (HSR) of Maastricht University. CAPHRI participates in the Netherlands School of Primary Care Research (CaRe).

The studies presented in chapters 3 to 5 of this thesis were financially supported by Zayed University, Dubai, United Arab Emirates.

Copyright (C) Cosette Fakih El Khoury, Maastricht, 2020

All rights reserved. No part of this thesis may be reproduced or transmitted in any form or by any means, electronic or mechanical, including photocopying, recoding on any information storage or retrieval system without permission from the author, or when appropriate, from the publisher of the publications.

ISBN: 978-94-6375-960-1

Printing: Ridderprint BV/ www.ridderprint.nl

Cover image: Sandy Abi Khalil 


\title{
An mHealth intervention for the dietary management of hemodialysis patients
}

\author{
DISSERTATION \\ to obtain the degree of Doctor at Maastricht University, \\ on the authority of the Rector Magnificus, Prof. Dr. Rianne M. Letschert, \\ in accordance with the decision of the Board of Deans, \\ to be defended in public \\ on Wednesday July 1,2020 , at 10:00 hours
}

by

Cosette Fakih El Khoury 


\section{Supervisors}

Prof. dr. J.M.G.A. Schols

Prof. dr. R.M.M. Crutzen

\section{Co-Supervisor}

Dr. R.J.G. Halfens

Dr. M. Karavetian (Zayed University, UAE)

\section{Assessment committee}

Prof. dr. N.K. de Vries (Chairman)

Prof. dr. J.P. Kooman

Prof. dr. W. Ibrahim (United Arab Emirates University, UAE)

Dr. F.J.S. Thilo (Bern University of Applied Sciences, Switzerland)

Dr. M.D. Spreeuwenberg 


\section{CONTENT}

General Introduction

The effects of dietary mobile apps on nutritional outcomes in adults with chronic diseases: a systematic review and meta-analysis

Dietary application for the management of patients with hemodialysis: a formative development study

A dietary mobile app for hemodialysis patients: a pilot study

Adequate management of phosphorus in hemodialysis using a dietary smartphone app: a pilot study

General discussion

Summary 127

Valorization

List of publications of the thesis

Acknowledgments

About the author 



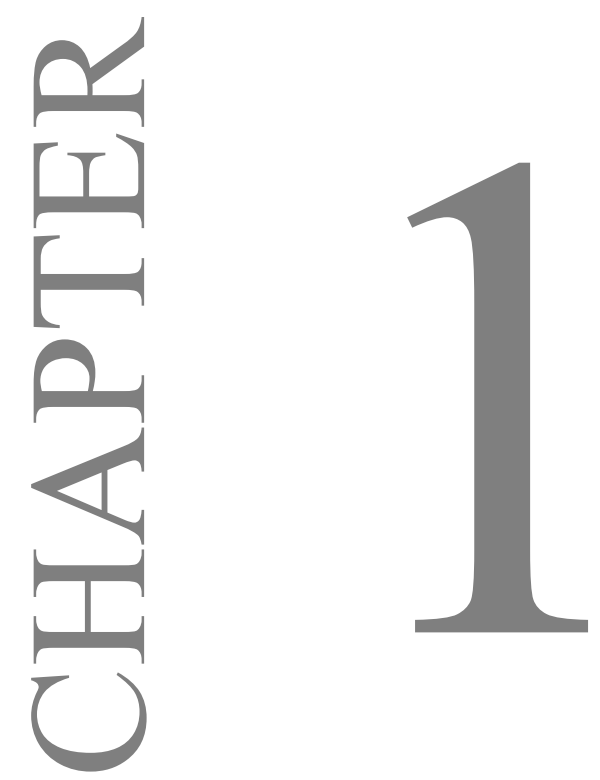

GENERAL INTRODUCTION 
Chronic kidney disease (CKD) represents a global health burden that is on the rise. Chronic kidney disease (CKD) consists of abnormalities in kidney structure or function for more than three months [1]. The most common underlying causes of chronic kidney disease (CKD) include diabetic nephropathy and cardiovascular diseases; other conditions that also lead to CKD include glomerulonephritis, genetic disorders, and urological condition, among others [2]. The incidence and prevalence of chronic kidney disease (CKD) have increased relevantly; for instance, the global incidence of CKD stages $1-5$ increased by $87 \%$ between 1990 and 2016 [3]. Across the Gulf Cooperation Council, data on the prevalence of hemodialysis patients varies between 640 per million to 1230 per million [4]. In the United Arab Emirates, it is estimated that about 760 persons per million were on dialysis in the year 2014 [4]. Moreover, global death and disabilities due to CKD have also increased in the past three decades. The trends of CKD are not in line with other non-communicable diseases, such as diabetes, cardiovascular diseases, and cancers, for which the number of deaths has decreased [3]. Thus, CKD burden appears on the rise, and given the increase in the aging population, the prevalence may increase even further in the future.

CKD management and control to minimize adverse outcomes necessitates a high level of involvement by patients. Treatment is perceived as a difficult and complex task that requires the adjustment of medications, lifestyle, and dietary adaptations, all of which require a high level of self-management [5]. Changing eating habits is challenging and requires an understanding of the behaviors along with the psychological, social, and economic factors that affect these behaviors [6]. In most chronic diseases, dietary and lifestyle changes are integral components of disease management [7]. Dietary interventions in CKD are intricate and require the adjustment of fluids, potassium, phosphorous, sodium while maintaining adequate protein and energy intakes to prevent malnutrition $[8,9]$. Accordingly, adherence to the renal diet is a challenging task for patients, especially during hemodialysis [10]; and dietary management requires behavioral changes that place a burden on the patient [11]. Additionally, CKD patients tend to be older and of lower socioeconomic backgrounds [5]. Lower levels of health literacy are also common among patients with CKD and are associated with adverse disease outcomes [12]. Education and self-management of CKD patients are, therefore, a cornerstone in the treatment with the potential to improve clinical outcomes [13].

Given the importance of education and self-management, eHealth has the potential to provide accessible, evidence-based education, along with self-management tools to support CKD patients and their healthcare practitioners, particularly dietitians in the achievement of behavioral changes and clinical goals.

More than a quarter century after the introduction of the Internet as a communication tool, eHealth transformed the healthcare sector and became an inescapable present and future for healthcare [14]. The World Health Organization (WHO) defined eHealth as "the costeffective and secure use of information and communication technology (ICT) in support of health and health-related fields, including healthcare services, health surveillance, health literature, and health education, knowledge and research" [15]. Another commonly utilized definition of eHealth states that "eHealth is an emerging field in the intersection of medical informatics, public health, and business, referring to health services and information 
delivered or enhanced through the Internet and related technologies. In a broader sense, the term characterizes not only a technical development, but also a state-of-mind, a way of thinking, an attitude, and a commitment for networked, global thinking, to improve health care locally, regionally, and worldwide by using information and communication technology"'[16].

The Internet has become an essential communication tool worldwide, and it is also commonly used for public health interventions [17]. The use of mobile devices has also increased over the years. At the end of 2015, there were more than seven billion mobile subscriptions worldwide, meaning about 120 mobile phone subscribers per 100 inhabitants [15]. In 2019, $97 \%$ of the world population lived within reach of a mobile cellular signal, and $82 \%$ lived within reach of mobile broadband access [18]. This rapid adoption of technology has contributed to the transformative nature of the healthcare sector, providing eHealth the opportunity to contribute to universal healthcare coverage [15]. Mobile health, also referred to as mHealth, falls under the broad definition of eHealth; however, it is specific to the use of mobile devices. mHealth has been defined by WHO as "the use of mobile devices such as mobile phones, patient monitoring devices, personal digital assistants (PDAs) and wireless devices-for medical and public health practice" [15].

The use of mobile phones includes the use of mobile applications, which have become indispensable in social and work settings [19]. The use of applications in the healthcare sector has opened new practice horizons across many fields, including dietetics. Given the prevalent number of smartphone owners, mHealth has the potential to deliver nutritional care to individuals and practitioners by providing the tools for continuous access to dietary interventions in health and disease.

We start this general introduction with an overview of mHealth in dietetics, followed by an overview of current knowledge related to the effectiveness of mobile apps in chronic diseases in general and in CKD. We then proceed by describing how components such as behavioral change theories, person-centeredness, development frameworks, and evidence can be integrated to develop feasible dietary interventions for hemodialysis patients using mHealth. The aim of this dissertation is to investigate the feasibility of using dietary mHealth interventions for hemodialysis patients.

\section{mHealth in dietetics}

The most popular dietary apps available to the public are nutrition and fitness apps, mainly focusing on self-monitoring of weight, food intake, and physical activity [20, 21]. Dietary apps could be supportive tools for patients, researchers, and dietetic practitioners. They could be used as monitoring tools that can capture eating patterns, dietary intakes, and lifestyle habits to support dietetics research [22]; however, they can also be used by dietetic practitioners as tools to support patient care [23].

Despite their potential impacts on dietary intakes, the usage of apps as a sole dietary intervention does not always yield changes on outcomes regarding anthropometric and biochemical measurements $[24,25]$. Several factors may impact the success of the 
intervention. For instance, user acceptability [26], quality of app content [27], the burden on users [28], in-app features, and use of behavioral models [29] are all factors that may influence app effectiveness.

Currently available in-app tracking devices require less engagement from users when monitoring physical activity as compared to food diaries. Whereas tracking physical activity is feasible through a wearable device, electronic food diaries require users to input information manually, which may be considered burdening [30]. A study comparing food diaries recorded on paper as compared diaries recorded on a computer or smartphone identified no difference in the accuracy of the food records. However, participants preferred computer or smartphone-based records as compared to the traditional paper-based food records [28]. Adherence and accuracy of self-monitoring are an essential component of dietary interventions. Thus, mobile apps may be alternatives to traditional paper-based food records that have higher acceptability and reduce burden [31].

Using mHealth as a tool to deliver dietetic care may have the potential to overcome current burdens, such as time, accessibility to a dietitian, and geographic burdens. Given the role of dietary interventions in chronic kidney disease, the potential benefits of mHealth in dietetics should be explored.

\section{mHealth in Chronic Kidney Disease (CKD)}

A systematic review of mobile health interventions on nutritional indicators in CKD published in 2015 [32] identified potential for dietary apps to benefit clinical outcomes in kidney disease. However, the review only found a few studies using personal digital assistants (PDA) rather than mobile apps and limited effectiveness [32]. A more recent review identified nine studies investigating the use of mobile apps in CKD. Only five studies reported in the review presented results on the effectiveness of the apps on dietary changes, and all found positive outcomes [33].

There are many renal apps available on app stores that are not necessarily supported by formative development studies. For example, Lambert et al., 2017 searched app stores for dietary apps addressed to CKD patients. They identified twenty-one apps for kidney diseases clustered into food compositions/recipes, educational apps, and self-monitoring apps; they also found one app addressed to healthcare professionals rather than patients [34]. However, only a few are developed based on a systematic approach that includes patients, practitioners, theories, frameworks, and evidence followed by an evaluation. Hence, many renal dietary apps are available for users to download, yet not many are based on formative development.

Most apps available consist of self-management tools that support patients in tracking food intakes, blood parameters, and physical parameters. Interventions, including education, are not as common; however, they are promising and have the potential to show improvement in outcomes such as blood pressure, glomerular filtration rate (eGFR), and sodium or albumin excretion [35].

Only a few apps consist of educational tools for patients; digital education is instead more available on websites and social media platforms (mainly YouTube videos) [5]. Most 
established materials are available through the National Kidney Disease Education Program (NKDEP), they are mainly in the English language, and they are based on American culturally specific foods [5]. Online education programs have been shown to improve knowledge and facilitate patient involvement in decision making. Dubin et al., 2019, for example, showed a significant improvement in the patient decision after a digital education intervention on renal replacement modalities. The capability of patients to make an informed decision improved from $32 \%$ being unable to decide to all patients being involved in decision making [13]. Patients, however, have shown interest in applications that are comprehensive and include educational materials, skill-building tools, and recipes [36, 37]; thus, future applications should consider the users' perspective and include such features in mHealth apps.

Despite available recommendations on dietary intakes, adherence to the renal diet remains very challenging $[9,10]$. Recent reviews in the field still emphasize the role of patient education as a critical component of behavioral interventions [35]. Unfortunately, the accuracy of the information available on apps is concerning; less than $50 \%$ of renal apps in commercial stores complied with evidence-based guidelines, and $71.4 \%$ did not report the sources of the information provided [34]. Additionally, commercial CKD apps available on app stores seem to lack the required functionalities to support patient self-management [38].

It seems essential, therefore, given the potential of mobile apps to support clinical outcomes in renal patients, that apps are developed following evidence-based guidelines. Partnerships between patients, healthcare practitioners, and developers contribute to better outcomes [39]; accordingly, a person-centered approach that involves all stakeholders (patients/caregivers, and healthcare practitioners) [40] should also be fostered from the beginning of the development process. Incorporation of theories of behavioral change guides the prediction and improvement of adherence in chronic kidney disease [41, 42], and therefore incorporation of theories would support in maximizing the benefits of such interventions. Incorporating all these components into an app would require a formative approach that systematically and iteratively addresses development.

\section{mHealth formative development}

Patient engagement is an essential component in the treatment of chronic disease; thus, any tool aimed at the improvement of clinical outcomes should be tailored to the patients' needs. Such reasoning, however, is not always implemented in the practice setting. Despite the rise and potential efficacy of mobile health in dietetics, patients do not use the tools as much as they could potentially [43]. This usage rate is not due to a lack of interest or lack of demand[44]; it is instead possibly due to lack of substantial evidence pertaining effectiveness [45], concerns about privacy and data security[46], and perceptions of healthcare professionals $[47,48]$. Additionally, the lack of attention to the user's perspectives during the design may highly contribute to low usage [43].

Technologies will only succeed if patients are ready, motivated, willing, capable of using them, and find them easy to use [49]. Many roadmaps, frameworks, and theories have addressed the development of mobile health solutions. We present below the IDEAS 
framework (Integrate, Design, Assess, and Share) [50] used in this thesis as it explicitly incorporates components of health behavior change, which better support the achievement of dietary goals. Other guiding frameworks are available; however, some focus only on core components of a successful mHealth intervention [51], others on design thinking for development [52], or evaluation only [53], while others highlight the involvement of a multidisciplinary approach[54]. Accordingly, we have opted in this thesis to use IDEAS as a guiding framework because it systematically combines behavioral theories, design thinking, evaluation, and dissemination [50].

\section{Development frameworks}

Early work guiding the development of software identified twelve key principles based on available theories, research, and experiences [55]. These principles focus on active user involvement in conceptualization, prototyping, and evaluation. The principles also include an iterative and incremental approach that involves a multidisciplinary team of experts [55].

A user-centered approach sets the user at the center of the development process; however, there is a great variety in how this approach is applied. The term person-based rather than user-centered depicts a more holistic approach that accommodates the inclusion of a multidisciplinary view that involves healthcare practitioners, caregivers, and other persons that may influence the user's usability [56]. Such a person-based approach may better capture the needs of the people who will use the technologies [56].

According to the person-based approach, grounding in theories of behavioral change is a fundamental component aimed at understanding the perspective of the persons that will use mHealth solutions. Qualitative interviews are also considered necessary tools to understand the perspective of the person [56]. This work is then complemented with evidence-based information to complete the development process [56].

The IDEAS framework sets a roadmap to the development, assessment, and dissemination processes by building upon the person-based approach and incorporating theory-based and evidence-based components. Accordingly, a comprehensive step-by-step framework guiding the development of mobile apps targeting behavioral change is detailed by the IDEAS framework [50]. The framework consists of 10 phases that are clustered into four categories, namely Integrate, Design, Assess and Share. Figure 1 depicts the IDEAS framework.

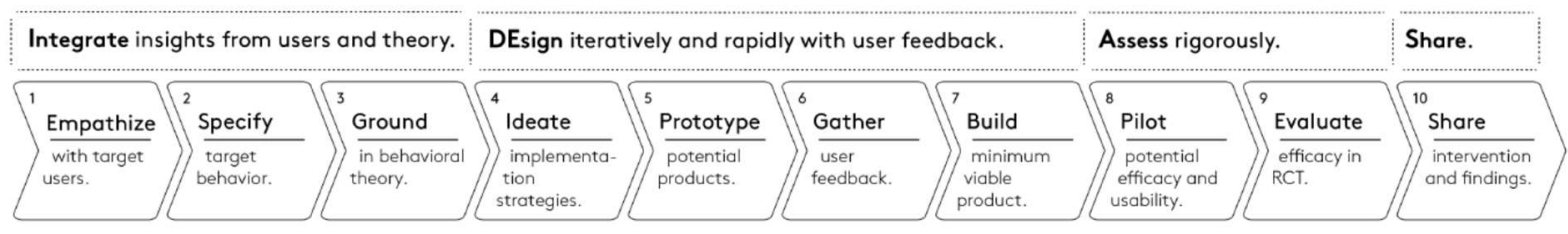

Figure 1: IDEAS Framework for the development of digital behavior change interventions [50]

IDEAS was used as a guiding framework in the development of this thesis project as it is a comprehensive framework applicable to behavioral interventions in dietetics. Chapter 3 
details the development process used for the Kidney Education for Lifestyle Application (KELA.AE), which was developed, and pilot tested in this thesis.

Theories and frameworks are useful tools to guide the development process. However, the implementation of each step may be problematic and is not often well defined. Despite having guidelines at each phase of the IDEAS framework well described, the actual implementation of the phases required several additional steps such as brainstorming, information gathering, and multidisciplinary involvement. Accordingly, across this thesis, we opted to incorporate the usage of core processes during the individual steps of the IDEAS framework. Core processes consist of practical ways aimed at finding answers to questions raised at various stages within a planning framework [57]. Core processes have been described as a component of Intervention Mapping [58]; however, they may be applied and used in many frameworks [57].

Given the complexity of real-life problems, behavioral theories are also iterative and evolve based on evidence from experimental studies [59]. Based on available knowledge that is topic, concept, and theory specific [57], we also incorporated multiple theories or constructs of theories of behavior change. The transtheoretical models (TTM) [60] and constructs from the Reasoned Action Approach (RAA) [61] were used during app development, and they were selected during brainstorming sessions as suggested by core processes.

\section{Objectives and outline of the thesis}

This thesis aims to provide an insight into the step-by-step development of an Arabic dietary mHealth intervention in chronic kidney disease based on an evidence-based, theory-based, and in-person approach. More specifically, in Chapter 2, we present a systematic review and meta-analysis of the available evidence on the effectiveness of dietary mobile app interventions in the context of chronic diseases. In Chapter 3, we describe in detail the stepby-step person-driven theory-based approach that was conducted to develop the selfmonitoring and educational dietary app KELA.AE for hemodialysis patients. The development followed the Integration, Design, Assessment, and Sharing (IDEAS) framework. Qualitative, semi-structured interviews with six hemodialysis patients and six healthcare practitioners (dietitians and nephrologists) were performed to assess the need for an app, the willingness to use an app, and features desired in an app. Chapters 4 and 5 present the results of a pilot study aimed to assess the feasibility of a dietary intervention using KELA.AE on dietary intakes and phosphorous management in hemodialysis patients. Chapter 6 discusses the methodological challenges, strengths, and limitations faced during the study; and implications of mHealth in dietetics for the professional practice and future research. 


\section{References}

1. Levin, A., et al., Kidney Disease: Improving Global Outcomes (KDIGO) CKD Work Group. KDIGO 2012 clinical practice guideline for the evaluation and management of chronic kidney disease. Kidney international supplements, 2013. 3(1): p. 1-150.

2. Evans, P.D. and M.W. Taal, Epidemiology and causes of chronic kidney disease. Medicine, 2011. 39(7): p. 402-406.

3. Xie, Y., et al., Analysis of the Global Burden of Disease study highlights the global, regional, and national trends of chronic kidney disease epidemiology from 1990 to 2016. Kidney international, 2018. 94(3): p. 567-581.

4. AlSahow, A., et al., Demographics and key clinical characteristics of hemodialysis patients from the Gulf Cooperation Council countries enrolled in the dialysis outcomes and practice patterns study phase 5 (2012-2015). Saudi Journal of Kidney Diseases and Transplantation, 2016. 27(7): p. 12.

5. Diamantidis, C.J. and S. Becker, Health information technology (IT) to improve the care of patients with chronic kidney disease (CKD). BMC nephrology, 2014. 15(1): p. 7.

6. Kelly, M.P. and M. Barker, Why is changing health-related behaviour so difficult? Public health, 2016. 136: p. 109-116.

7. Pereira, R.A., et al., Diet in Chronic Kidney Disease: an integrated approach to nutritional therapy. Revista da Associação Médica Brasileira, 2020. 66: p. s59-s67.

8. Kopple, J.D., National kidney foundation K/DOQI clinical practice guidelines for nutrition in chronic renal failure. American journal of kidney diseases, 2001. 37(1): p. S66-S70.

9. Luis, D., et al., Dietary quality and adherence to dietary recommendations in patients undergoing hemodialysis. Journal of Renal Nutrition, 2016. 26(3): p. 190-195.

10. Hayashi, A., et al., Testing the feasibility and usability of a novel smartphone-based selfmanagement support system for dialysis patients: a pilot study. JMIR research protocols, 2017. 6(4): p. e63.

11. D’Alessandro, C., G.B. Piccoli, and A. Cupisti, The "phosphorus pyramid": a visual tool for dietary phosphate management in dialysis and CKD patients. BMC nephrology, 2015. 16(1): p. 9.

12. Jain, D. and J.A. Green, Health literacy in kidney disease: Review of the literature and implications for clinical practice. World journal of nephrology, 2016. 5(2): p. 147.

13. Dubin, R. and A. Rubinsky, A Digital Modality Decision Program for Patients With Advanced Chronic Kidney Disease. JMIR formative research, 2019. 3(1): p. e12528. 
14. Charani, E., et al., Do smartphone applications in healthcare require a governance and legal framework? It depends on the application! BMC medicine, 2014. 12(1): p. 29.

15. Organization, W.H., Global diffusion of eHealth: making universal health coverage achievable. 2017: World Health Organization.

16. Eysenbach, G., What is e-health? J Med Internet Res, 2001. 3(2): p. e20.

17. Bennett, G.G. and R.E. Glasgow, The delivery of public health interventions via the Internet: actualizing their potential. Annual review of public health, 2009. 30: p. 273292.

18. Bogdan-Martin, D., Measuring digital development Facts and figures 2019, I.T.D. Bureau, Editor. 2019, International Telecommunication Union Geneva Switzerland.

19. Papadopoulos, H., V.B. Sheth, and M. Wurst, Comparison of US and EU regulatory approaches to mobile health apps: use cases of myVisionTrack and USEFIL. mHealth Regulatory Environments, 2013: p. 27.

20. Hingle, M. and H. Patrick, There are thousands of apps for that: navigating mobile technology for nutrition education and behavior. Journal of nutrition education and behavior, 2016. 48(3): p. 213-218. e1.

21. Higgins, J.P., Smartphone applications for patients' health and fitness. The American journal of medicine, 2016. 129(1): p. 11-19.

22. Gill, S. and S. Panda, A smartphone app reveals erratic diurnal eating patterns in humans that can be modulated for health benefits. Cell metabolism, 2015. 22(5): p. 789-798.

23. Rusnak, S. and P. Charney, Position of the Academy of Nutrition and Dietetics: Nutrition Informatics. Journal of the Academy of Nutrition and Dietetics, 2019. 119(8): p. 13751382 .

24. Helle, C., et al., Evaluation of an eHealth intervention aiming to promote healthy food habits from infancy-the Norwegian randomized controlled trial Early Food for Future Health. International Journal of Behavioral Nutrition and Physical Activity, 2019. 16(1): p. 1 .

25. Gonzalez-Sanchez, J., et al., Using a smartphone app in changing cardiovascular risk factors: A randomized controlled trial (EVIDENT II study). International journal of medical informatics, 2019. 125: p. 13-21.

26. Hutchesson, M.J., et al., Be positive Be health e: development and implementation of a targeted e-health weight loss program for young women. TELEMEDICINE and eHEALTH, 2016. 22(6): p. 519-528.

27. Lambert, K., et al., Evaluation of the quality and health literacy demand of online renal diet information. Journal of human nutrition and dietetics, 2017. 30(5): p. 634-645. 
28. Hutchesson, M.J., et al., Self-monitoring of dietary intake by young women: online food records completed on computer or smartphone are as accurate as paper-based food records but more acceptable. Journal of the Academy of Nutrition and Dietetics, 2015. 115(1): p. 87-94.

29. Azar, K.M., et al., Mobile applications for weight management: theory-based content analysis. American journal of preventive medicine, 2013. 45(5): p. 583-589.

30. König, L.M., et al., Describing the Process of Adopting Nutrition and Fitness Apps: Behavior Stage Model Approach. JMIR mHealth and uHealth, 2018. 6(3): p. e55.

31. Hughes, D.C., et al., BALANCE (Bioengineering Approaches for Lifestyle Activity and Nutrition Continuous Engagement): developing new technology for monitoring energy balance in real time. Journal of diabetes science and technology, 2010. 4(2): p. 429-434.

32. Campbell, J. and J. Porter, Dietary mobile apps and their effect on nutritional indicators in chronic renal disease: a systematic review. Nephrology, 2015. 20(10): p. 744-751.

33. Kosa, S.D., et al., Nutritional mobile applications for CKD patients: systematic review. Kidney international reports, 2019. 4(3): p. 399-407.

34. Lambert, K., et al., Should We Recommend Renal Diet-Related Apps to Our Patients? An Evaluation of the Quality and Health Literacy Demand of Renal Diet-Related Mobile Applications. Journal of Renal Nutrition, 2017. 27(6): p. 430-438.

35. Evangelidis, N., et al., Lifestyle behaviour change for preventing the progression of chronic kidney disease: a systematic review. BMJ open, 2019. 9(10).

36. LeRouge, C., et al., Design Guidelines for a Technology-Enabled Nutrition Education Program to Support Overweight and Obese Adolescents: Qualitative User-Centered Design Study. Journal of medical Internet research, 2019. 21(7): p. e14430.

37. Biediger-Friedman, L., et al., User-centered design of a Texas WIC app: a focus group investigation. American journal of health behavior, 2016. 40(4): p. 461-471.

38. Lee, Y.-L., et al., Mobile health to maintain continuity of patient-centered care for chronic kidney disease: content analysis of apps. JMIR mHealth and uHealth, 2018. 6(4): p. e10173.

39. Lewis, R.A., et al., Identifying Mobile Applications Aimed at Self-Management in People With Chronic Kidney Disease. Canadian Journal of Kidney Health and Disease, 2019. 6: p. 2054358119834283.

40. Fakih El Khoury, C., et al., Dietary Application for the Management of Patients with Hemodialysis: A Formative Development Study. Healthcare informatics research, 2019. 25(4): p. 262-273. 
41. Havas, K., C. Douglas, and A. Bonner, Meeting patients where they are: improving outcomes in early chronic kidney disease with tailored self-management support (the CKD-SMS study). BMC nephrology, 2018. 19(1): p. 279.

42. Chironda, G., B. Bhengu, and A. Manwere, Models and theories of care applicable to predicting and improving adherence behaviours among Chronic Kidney Disease (CKD) patients. Rwanda Journal of Medicine and Health Sciences, 2019. 2(1): p. 48-58.

43. Birnbaum, F., et al., Patient engagement and the design of digital health. Academic emergency medicine: official journal of the Society for Academic Emergency Medicine, 2015. 22(6): p. 754.

44. Ranney, M.L., et al., Emergency department patients' preferences for technology-based behavioral interventions. Annals of emergency medicine, 2012. 60(2): p. 218-227. e48.

45. El Khoury, C.F., et al., The Effects of Dietary Mobile Apps on Nutritional Outcomes in Adults with Chronic Diseases: A Systematic Review. Journal of the Academy of Nutrition and Dietetics, 2019.

46. Zhou, L., et al., Barriers to and Facilitators of the Use of Mobile Health Apps From a Security Perspective: Mixed-Methods Study. JMIR mHealth and uHealth, 2019. 7(4): p. e11223.

47. Daniel, F., et al., Patient-physician communication in the era of mobile phones and social media apps: cross-sectional observational study on Lebanese physicians' perceptions and attitudes. JMIR medical informatics, 2018. 6(2): p. e18.

48. Garnweidner-Holme, L., et al., Health Care Professionals' Attitudes Toward, and Experiences of Using, a Culture-Sensitive Smartphone App for Women with Gestational Diabetes Mellitus: Qualitative Study. JMIR mHealth and uHealth, 2018. 6(5): p. e123.

49. Wake, D.J., et al., MyDiabetesMyWay: an evolving national data driven diabetes selfmanagement platform. Journal of diabetes science and technology, 2016. 10(5): p. 10501058 .

50. Mummah, S.A., et al., IDEAS (Integrate, Design, Assess, and Share): a framework and toolkit of strategies for the development of more effective digital interventions to change health behavior. Journal of medical Internet research, 2016. 18(12): p. e317.

51. Waterlander, W., et al., Development of an evidence-based mHealth weight management program using a formative research process. JMIR mHealth and uHealth, 2014. 2(3): p. e18.

52. Ludden, G.D., et al., How to increase reach and adherence of web-based interventions: a design research viewpoint. Journal of medical Internet research, 2015. 17(7): p. e172. 
53. Brown III, W., et al., Assessment of the Health IT Usability Evaluation Model (HealthITUEM) for evaluating mobile health (mHealth) technology. Journal of biomedical informatics, 2013. 46(6): p. 1080-1087.

54. Van Velsen, L., J. Wentzel, and J.E. Van Gemert-Pijnen, Designing eHealth that matters via a multidisciplinary requirements development approach. JMIR research protocols, 2013. 2(1): p. e21.

55. Gulliksen, J., et al., Key principles for user-centred systems design. Behaviour and Information Technology, 2003. 22(6): p. 397-409.

56. Yardley, L., et al., The person-based approach to intervention development: application to digital health-related behavior change interventions. Journal of medical Internet research, 2015. 17(1): p. e30.

57. Ruiter, R.A., R. Crutzen, and G. Kok, Core Processes for Developing Theory-and Evidence-Based Interventions. 2018.

58. Eldredge, L.K.B., et al., Planning health promotion programs: an intervention mapping approach. 2016: John Wiley \& Sons.

59. Peters, G.-J.Y., M. De Bruin, and R. Crutzen, Everything should be as simple as possible, but no simpler: towards a protocol for accumulating evidence regarding the active content of health behaviour change interventions. Health Psychology Review, 2015. 9(1): p. 1-14.

60. Prochaska, J.O. and W.F. Velicer, The transtheoretical model of health behavior change. American journal of health promotion, 1997. 12(1): p. 38-48.

61. Fishbein, M., A reasoned action approach to health promotion. Medical Decision Making, 2008. 28(6): p. 834-844. 

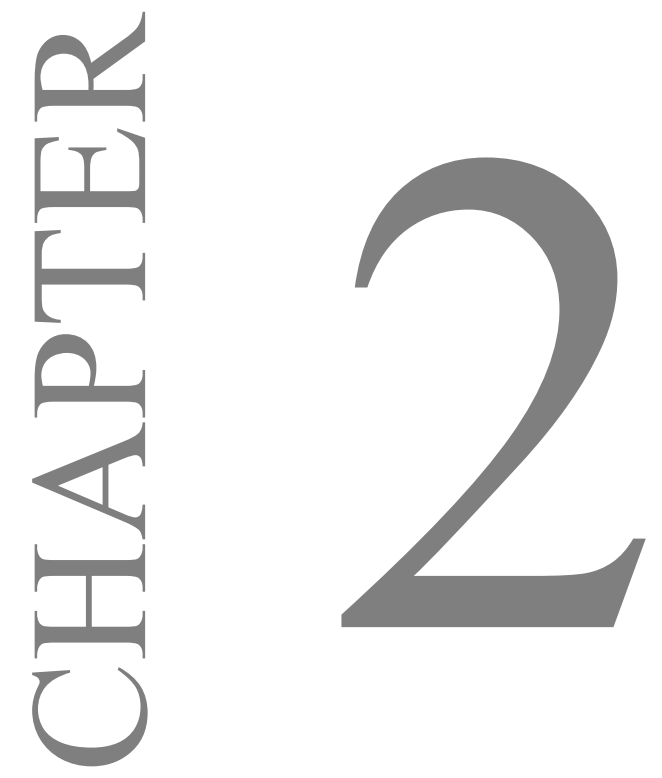

THE EFFECTS OF DIETARY MOBILE APPS ON NUTRITIONAL OUTCOMES IN ADULTS WITH CHRONIC DISEASES: A SYSTEMATIC REVIEW AND META-ANALYSIS

Cosette Fakih El Khoury, Mirey Karavetian, Ruud J. G. Halfens, Rik Crutzen, Lama Khoja, Jos M. G. A. Schols 


\begin{abstract}
Background: Dietary interventions are effective prevention and treatment strategies for chronic diseases; however, they require extensive commitment, time, and resources. Dietary mobile apps have gained popularity and are thus being incorporated into dietary management.

Objective: The aim of this review is to assess the effects of the use of dietary mobile apps on nutritional outcomes in adults with chronic diseases.

Methods: A systematic review was conducted following PRISMA guidelines using MEDLINE, PubMed, Embase, and CINAHL databases. The protocol was registered on PROSPERO. Intervention studies evaluating the nutritional outcomes of dietary apps, published in English between January 1, 2007, and November 15, 2017, were included. The methodological quality of included articles was assessed via the Academy of Nutrition and Dietetics' Quality Criteria Checklist: Primary Research. Heterogeneity was confirmed using the I2 index and a random-effects meta-analysis was performed for randomized controlled trials. Estimates of the pooled mean difference were calculated for app usage as compared to no app usage.
\end{abstract}

Main Outcomes Measure: Nutrition outcomes categorized as food/nutrition-related, anthropometric measurements, pertinent clinical/biochemical data, and nutrition-focused physical findings were extracted from the included intervention studies.

Results: Upon completion of the searches, 18,649 articles were identified, and data were extracted from 22 articles. Pooled estimates showed a significantly greater decrease in weight $(-2.45 \mathrm{Kg}, 95 \%$ CI -3.33 to $-1.58 ; \mathrm{p}<0.001 ; \mathrm{I} 2=96.2 \%, 95 \%$ CI $95 \%$ to $97 \%)$, waist circumference $(-2.54 \mathrm{~cm}, 95 \% \mathrm{CI}-3.34$ to $-1.73 ; \mathrm{p}<0.001 ; \mathrm{I} 2=88.3 \%, 95 \%$ CI $67 \%$ to $96 \%)$, and energy intake (-149.52 Cal, 95\% CI $-215.78,-83.27 ; \mathrm{p}<0.001 ; \mathrm{I} 2=0 \% \mathrm{CI} 0 \%$ to $90 \%)$ when an app was used as compared to control.

Conclusions: The findings of this systematic review and meta-analysis indicate that dietary mobile apps are effective self-monitoring tools, and that their use results in positive effects on measured nutritional outcomes in chronic diseases, especially weight loss.

Keywords: dietary mobile apps, mobile apps, nutrition intervention, chronic disease, nutritional outcomes 


\section{INTRODUCTION}

The prevalence of chronic diseases is reaching a major epidemic level. Globally, chronic diseases account for $68 \%$ of all deaths, most of which are caused by cardiovascular diseases, diabetes, cancer, and chronic respiratory diseases [1].

Dietary interventions are among the most effective and affordable prevention strategies where chronic diseases are concerned [2]. In addition, they are cost-effective, evidence-based therapeutic measures in the management of many chronic diseases $[3,4]$. Positive outcomes are attributable to nutrition education or counseling interventions delivered by dietitians (rather than other professionals) $[5,6,7]$, and changes in dietary intake [8]. Recent systematic reviews have also concluded that dietetic consultations and medical nutrition therapy are successful in reducing the risk of diabetes development, ameliorating blood glucose and glycated hemoglobin in patients with diabetes, promoting weight loss in obesity, and improving compliance to dietary guidelines $[9,10]$. Additionally, such reviews reveal cost-saving effects in the treatment of patients with obesity and diabetes [11]. The effectiveness of dietary interventions has been supported in different fields, including chronic kidney disease [5, 7, 12], nephrolithiasis [8], obesity, and diabetes [6, 13], among other areas [4, 14, 15].

Patients suffering from chronic diseases are often required to make changes in their diets and lifestyles. Generally, dietary interventions comprise in-person counseling sessions, which require skilled and well-trained professionals [16]. Multiple individualized [17], culturally sensitive [18] sessions, each lasting at least 30 minutes, are needed for effective outcomes [19]. Thus, dietary interventions involve both time and resources [9]. In-person sessions, taking place in clinical settings, require commitment and, if missed, may not be made up for by the patients. For instance, the IDEA study reported that only $9.5 \%$ of the patients enrolled in their trial made up for missed sessions [20]. Adherence to multiple counseling sessions seems challenging for patients; a systematic review described a dropout rate of up to $35 \%$ of patients enrolled in dietetic consultation interventions [9]. Patient empowerment has been suggested as a solution to compliance challenges [21] as it changes the traditional passive relationship between the patient and healthcare providers to a more interactive one [22]. Smartphones may offer relatively inexpensive options allowing patient engagement and empowerment, selfmonitoring, and communication with healthcare providers $[23,24]$. Moreover, patients have a desire for interventions that require less in-person contact with providers $[21,25,26]$. The convenience of access to self-management, information, and healthcare providers is a potential benefit of mobile app platforms that supports an interactive relationship between the patient and the entire healthcare system [21,22]. Mobile apps that provide tools intended to facilitate 
nutritional care via smartphone technologies provide patients with more autonomy, thus empowering them and offsetting patient disengagement $[27,28]$.

The number of smartphone users has increased dramatically; smartphone ownership is reported to be $43 \%$ of adults globally, and $72 \%$ in the United States [29]. Health-related applications have gained popularity as tools to support users in the change of a target behavior [30]. A selection of dietary apps is available for download on mobile phones. These include food composition databases; exercise, meal behavior, and food intake diaries; and nutrition education. Some of these target healthy people, while others target patients suffering from chronic diseases. These apps are often free or available for download at very low cost. Their quality and effectiveness, however, are not yet well established, which makes their assessment necessary given the rapid development of mobile technology.

Systematic reviews to date have focused on healthy participants or examined the effects of dietary apps on diet improvement [31]. Two systematic reviews have investigated nutritional outcomes in chronic diseases; however, they targeted a single disease each (diabetes and chronic renal disease), and included any mobile technology-based intervention, such as personal digital assistants, without focusing on smartphone apps [32,33]. Most reviews were inconclusive, with the authors recommending further research in the area to demonstrate possible benefits $[33,32,24]$.

Given that the commercial apps available in app stores are being developed faster than they are being evaluated and that they are highly accessible to the public [27], it seems desirable to assess their impact on nutritional outcomes. Accordingly, this review will focus on the effects of the use of dietary mobile apps on nutritional outcomes in adults with chronic diseases.

\section{MethodS}

This systematic review protocol employed the standard methods established by PRISMA [34] across all stages, including the formulation of the research question. The review has been registered on the PROSPERO International prospective register of systematic reviews (registration ID: CRD42017056923

http://www.crd.york.ac.uk/PROSPERO/display_record.asp?ID=CRD42017056923).

\section{SEARCH STRATEGY}

A systematic search was conducted on November 15, 2017, in a total of four databases comprising MEDLINE (using Ovid), PubMed, Embase (using Ovid), and CINAHL (using EBSCO). Three main concepts were searched: mobile applications, nutrition intervention, and chronic diseases. The search strategy used free-text words in addition to controlled vocabulary terms (such as MeSH headings for MEDLINE and PubMed) for each concept. A medical 
librarian experienced with systematic reviews approved the search strategy. Literature in English published between January 1, 2007 and November 15, 2017, was searched. This time frame was selected because mobile apps began appearing on smartphones in 2008 [35]; thus, pioneer emerging research during the previous year was included. Duplicate articles were removed, and references to relevant articles were hand-searched to identify possible additional papers for inclusion. The full search strategy is available on https://www.crd.york.ac.uk/PROSPEROFILES/56923_STRATEGY_20170514.pdf.

\section{ELIGIBILITY CRITERIA}

Studies' eligibility was determined based on a preset list of inclusion and exclusion criteria as detailed in Table 1. Intervention studies published in English, meeting the eligibility criteria, were selected during the screening process. Only randomized controlled trials (RCTs) reporting the measured outcomes have been included in the meta-analysis.

\section{STUDY SELECTION}

Two reviewers (C. K., L.K.) autonomously screened the titles and abstracts to determine the studies that met the inclusion criteria. A conservative approach was used and in case of hesitation, the study was included for screening in the next phase. After potentially eligible studies were agreed upon, the full texts were obtained. Two reviewers (C.K., M.K.) then independently read the complete articles. Agreement between the two reviewers was $95.8 \%$ $(\mathrm{k}=0.91 \pm 0.06,95 \%$ confidence interval (CI) 0.78 to 1.00 ; only one article was disagreed upon. Accordingly, discrepancies were resolved through discussion and a consensus was reached between the two reviewers. 
Table 1: Criteria for inclusion and exclusion of articles for a systematic review assessing the effectiveness of dietary mobile applications in chronic diseases on nutritional outcomes in adults

\begin{tabular}{|c|c|c|}
\hline Criteria & Inclusion & Exclusion \\
\hline Participants/ population & $\begin{array}{l}\text { - Adults ( } \geq 18 \mathrm{yrs}) \text { with } \\
\text { chronic disease }\end{array}$ & $\begin{array}{l}\text { - Healthy subjects } \\
\text { - } \text { Participants with healthy } \\
\mathrm{BMI}^{1}\end{array}$ \\
\hline Intervention(s)/exposure(s) & $\begin{array}{l}\text { - Use of a smartphone app } \\
\text { and, } \\
\text { - Dietary/nutritional } \\
\text { Intervention }\end{array}$ & $\begin{array}{l}\text { - Use of tablets, web-based } \\
\text { interventions, } \mathrm{PDA}^{2} \text { or } \\
\text { computer only } \\
\text { - Use of } \mathrm{SMS}^{3} \text { or phone } \\
\text { calls only } \\
\text { - No dietary/nutritional } \\
\text { intervention } \\
\text { - Interventions including } \\
\text { exercise only }\end{array}$ \\
\hline Comparator(s)/ control & $\begin{array}{l}\text { - Any comparison } \\
\text { - Presence of } \\
\text { control/absence of control }\end{array}$ & - None \\
\hline Outcome(s) & $\begin{array}{l}\text { Include at least one } \\
\text { nutritional outcome defined } \\
\text { as: } \\
\text { - food-/nutrition-related } \\
\text { outcomes, } \\
\text { - anthropometric } \\
\text { measurement outcomes, } \\
\text { - pertinent } \\
\text { clinical/biochemical data } \\
\text { outcomes, } \\
\text { - nutrition-focused physical } \\
\text { findings }\end{array}$ & $\begin{array}{l}\text { Studies } \\
\text { reporting/examining: } \\
\text { - applications only aimed at } \\
\text { health-care professionals } \\
\text { - application development } \\
\text { only } \\
\text { - satisfaction, learnability, } \\
\text { memorability, errors and } \\
\text { cognitive loads of mobile } \\
\text { applications only }\end{array}$ \\
\hline Other & $\begin{array}{l}\text { - English language } \\
\text { - Published between } 2007 \\
\text { and } 2017 \\
\text { - Intervention studies }\end{array}$ & $\begin{array}{l}\text { Conference abstracts, } \\
\text { reviews, letters to editor, } \\
\text { Opinion papers }\end{array}$ \\
\hline
\end{tabular}

${ }^{1}$ BMI: Body Mass Index, healthy BMI refers to a BMI range between 18.5 and $24.9 \mathrm{~kg} / \mathrm{m}^{2}$

2 PDA: Personal Digital Assistant

${ }^{3}$ SMS: Short Message Service or text message

\section{DATA EXTRACTION}

A preliminary synthesis was performed to produce descriptive paragraphs summarizing the key findings of each of the articles included. Articles were then grouped based on the intervention type (mobile apps as the sole intervention, multiple-arm interventions, and counseling interventions supported by mobile apps). The results were tabulated using an extraction form, 
approved in advance by two authors (C.K., M.K.), which was developed specifically for this review. Information extracted included study design, participant characteristics, chronic disease addressed, and nutrition outcomes categorized based on the nutrition care process (NCP). The Academy of Nutrition and Dietetics adapted the nutrition care process as a standardized model for the provision of nutrition care; it aims to improve the consistency and quality of individualized care [36]. Nutrition outcomes, categorized according to the nutrition care process, are food/nutrition-related outcomes, anthropometric measurements, pertinent clinical/biochemical data, and nutrition-focused physical findings. Relevant results retrieved from the synthesis of the descriptive paragraphs and tabulated data were reviewed and compiled into a results' table by one author (C.K.).

\section{QUALITY ASSESSMENT}

The methodological quality of included articles was assessed by the reviewers (C.K., M.K.) via the Academy of Nutrition and Dietetics' Quality Criteria Checklist: Primary Research [37]. This tool consists of a series of questions that address studies specifically on nutrition and dietetics. The checklist assesses the study quality based on 10 criteria including the research question, selection process, group comparability, withdrawals, blinding, interventions, outcomes, statistical analysis, conclusions, and conflicts of interest. The scale provided by the checklist, which relies on "yes" or "no" answers, was used to classify articles as negative, neutral, or positive. According to the checklist, when an article receives a "no" to most questions (six or more), it should be designated as negative. If most questions receive a "yes" (questions pertaining to selection process, group comparability, interventions, and outcomes must receive a "yes" with at least one additional "yes"), the article should be designated as positive. A neutral rating is provided to articles that fall between the above-described ratings. The quality assessment was performed by each author separately with blinding to each other's answers; overall agreement was $88.2 \% \quad(\mathrm{k}=0.72 \pm 0.19$, 95\% CI 0.35 to 1.00$)$. Through discussion and consensus, the reviewers identified and resolved disagreements and classified the articles as minus/negative, neutral, or plus/positive. This was done in accordance with the guidelines of the Quality Criteria Checklist present in the Academy of Nutrition and Dietetics Evidence Analysis Manual [37].

\section{STATISTICAL ANALYSIS}

Effect sizes, which were not reported, were calculated as Cohen's $d$ [38, 39] using the mean and standard deviations of measured outcomes. For the meta-analysis, estimates of the pooled mean differences were calculated when at least two or more RCTs reported data for the same outcome. Weighted mean between-group difference for app usage as compared to no app usage for changes in weight, body mass index (BMI), waist circumference, energy intake, 
fruit/vegetable intake, and Hbalc (95\% CIs) were calculated. A random-effects model with DerSimonian and Laird's technique [40] was used to account for heterogeneity. The statistical heterogeneity test I2 index was used to determine heterogeneity [41]. Changes in outcome parameters were calculated as the difference between baseline parameters and end-of-treatment parameters related to app usage or control (no app usage). For studies without variance data, the variance was calculated from CIs or ranges [42-45]. Whenever an RCT comprised several arms, only the arm using an app was included and compared to the arm without an app. Studies that incorporated an app in all experimental arms were not included in the meta-analysis [46]. Forest plots were generated using Stata Statistical Software, release 11(TX: StataCorp) [47]. Publication bias tests were not performed due to the small number of studies included in the meta-analysis [48].

\section{RESULTS}

Upon completion of the searches, 18,649 articles were identified, with 12,125 left after the removal of duplicates. Identified articles were subjected to a title and abstract screening step, which resulted in 59 articles. Then, these were retrieved and reviewed as full text. Twentytwo articles were included in the qualitative assessment. Figure 1 depicts the flowchart of the selection process. 
18,649 articles were identified through the database search No additional articles were identified from reference lists search
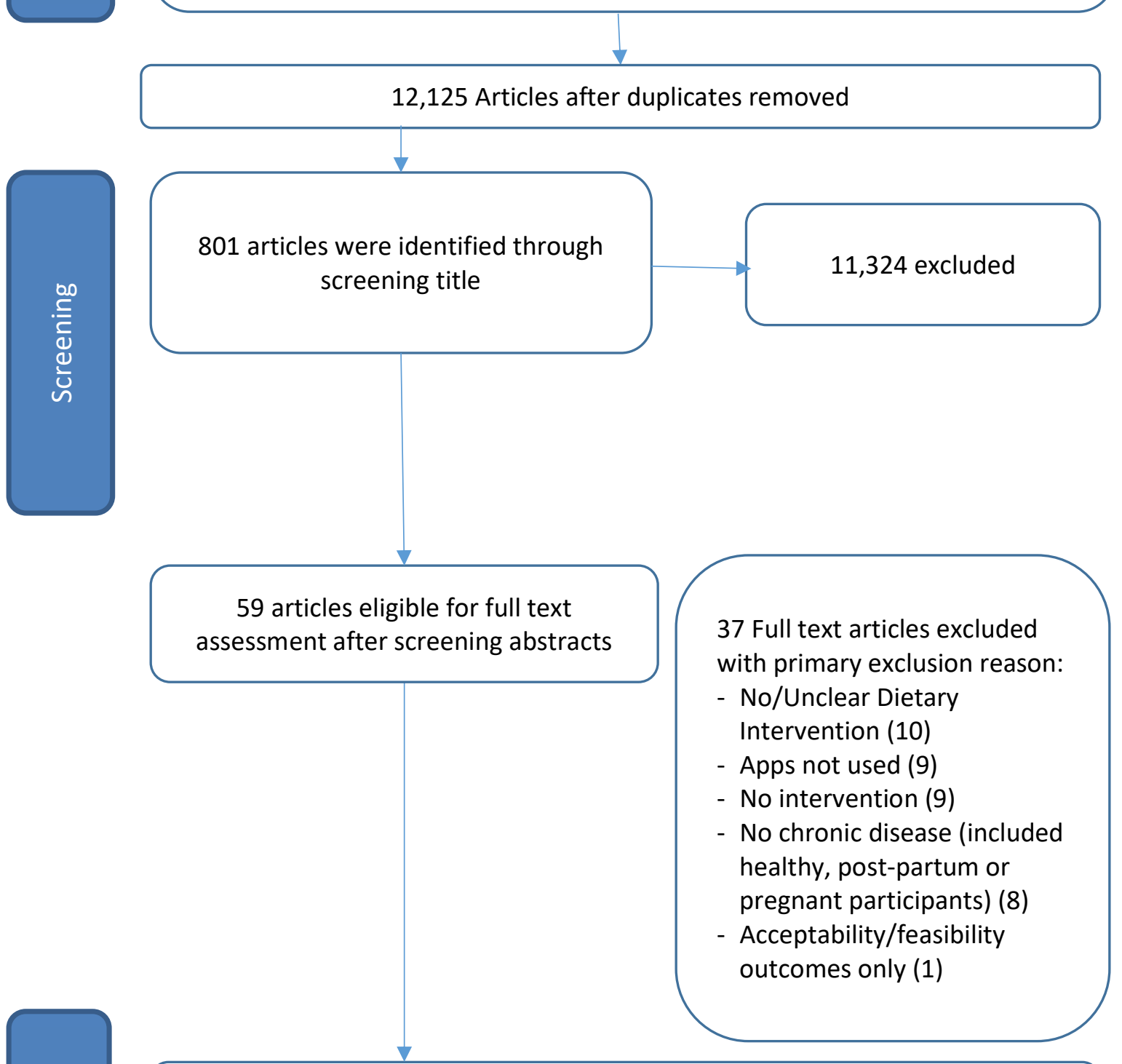

22 articles were included in the qualitative analysis after full text screening No additional articles were included from reference lists search

Figure 1: Four phase flow diagram following PRISMA statement for the article selection process of a systematic review assessing the effectiveness of dietary mobile applications on nutritional outcomes in adults with chronic diseases 


\section{DESCRIPTION OF INCLUDED ARTICLES}

The evaluated applications mainly targeted obesity [43, 46, 49-58] and diabetes (Type 1 and Type 2) [44, 45, 59-61]. One intervention addressed cardiovascular disease [62], one addressed patients with overweight/obesity at risk of breast cancer [63], and another one targeted patients with overweight/obesity and endometrial and breast cancer [64]. Sixteen out of 22 [42-46, 49, 51-54, 56-58, 61-63, 65] articles were RCTs or pilot RCTs with two or three arms. Three included a single-arm design with pre-post assessments [55, 60, 64]. Most interventions took place in the United States [42-44, 52, 53, 55-57, 63-65], two in the United Kingdom [49, 58], two in Korea [59, 60], one in Australia [61], and one in Norway [45]. The majority of included studies reported anthropometric outcomes [42-45, 49, 52, 53, 55-60, 63, 64], six studies reported biochemical data, medical tests, and procedures [44, 45, 55, 56, 60, $61]$, and 10 studies reported food/nutrition-related outcomes [42, 43, 45, 52, 53, 59-61, 63, $65]$.

Intervention durations mostly varied between three and nine months, with one month-long study [59] and one that lasted a year [45]. Most of the studies reported educational app usage or tracking/logging days through the app [42, 43, 45, 46, 49, 50, 52, 54-58, 60, 61, 65]. Both the post-hoc analyses identified by this search investigated the effects of increased app usage. Better improvements in nutritional outcomes were observed with increased frequency of app usage $[49,53]$. Participants consisted of male and female adults between 18 and 80 years of age. One article only included male participants (truck drivers [50]) and two articles only included female participants (at risk of breast cancer and with a history of breast or endometrial cancer [64]).

\section{Mobile Apps Evaluated}

Eleven studies evaluated commercial apps (apps available to any user on app stores) [42, 43, $46,50,52-54,56,57,63,64]$, while 10 studies evaluated apps developed for a specific intervention [44, 45, 49, 55, 58-62, 65]. Apps mainly focused on dietary and physical activity self-monitoring [42-46, 49-54, 56-59, 61, 63-65] and two consisted of in-app education [55, 62]. One of the apps included both features60. In 14 interventions, authors reported the use of a behavioral theory or its constructs [42-45, 49, 51-54, 56, 58, 63-65]. Behavioral theories were either embedded into the app's features or the interventional trial. Among studies incorporating behavioral theories, some had mobile apps as the sole intervention [50, 51, 53-56, 58-62, 65], while some multiple-arm interventions included them as the sole intervention in one arm [43, $45,49,57]$. The behavioral theories used included the Social Cognitive Theory [43, 51-54, 64], Transtheoretical Model [45], and the Theory of Planned Behavior [64]. Some studies used constructs of behavioral theories, such as self-monitoring, goal setting, and self-efficacy [42, 
44, 49, 56-58, 65]. Six studies used mobile apps as support for an intervention that consisted of in-person or phone counseling sessions $[42,44,46,52,63,64]$. Data extracted from the studies are reported in Table 2.

\section{QUALITY ASSESSMENT}

Most articles were rated as neutral and seven received a positive rating [42, 44, 49, 51, 54, 56, 62]. Common reasons for a neutral rating included pilot designs [43, 57, 64], lack of power analysis [43, 46, 49, 50, 55], convenience sampling, and lack of blinding [59]. Among the trials that were rated positively, only one failed to find favorable outcomes [56]. Quality assessment results are reported in Table 3.

\section{MOBILE APPS AS SOLE INTERVENTION}

Studies using mobile apps as the sole intervention mainly evaluated their accessibility compared to usual care or a different technology (bite counter)[54]. Three interventions used a pre-post assessment $[50,55,60]$ and two consisted of a post-hoc analyses of app users of an original trial $[53,58]$. Among the seven studies assessing anthropometric measurements in this category, only two found no significant changes $[56,59]$ in any nutritional outcome; however, one of these reported a decrease in app usage over time [56] and the other did not report app usage [59]. All other studies found apps to be effective, for instance, one that found a significant medium effect size, decrease in weight $(-6.12 \mathrm{~kg}, \mathrm{p}=0.005)$, and waist circumference $(-7.2 \mathrm{~cm}, \mathrm{p}=0.005)$ as compared to baseline [55]. Another study [54] found app usage to lead to significantly greater weight loss as compared to a wearable bite counter ($6.8 \pm 0.8 \mathrm{~kg}$, large effect size, $\mathrm{p}=0.001$ ), with weight loss being significantly correlated to higher app usage days $(\mathrm{r}=-0.33, \mathrm{P}<0.01)$. Both post-hoc analyses $[53,58]$ found significant improvements in anthropometric measurements among the app users. One of these post-hoc analyses [53] identified that using the physical activity self-monitoring feature of the app led to a significant and large impact on BMI and weight loss among app users as compared to non-app users (large effect sizes, $p=0,02, p=0.01$ respectively). Eight studies looked at food/nutrition-related history and all found significant improvements either in a specific food or food group (fruit [50], vegetable [50,51], or salt [62] intake), energy intake [53,54] (decreased energy intake), attitude and/or behavior scores [59], or self-reported dietary scores on the Summary of Diabetes Self-Care Activities questionnaire (SDSCA) [60, 61] (SDSCA scores, $\mathrm{p}<0.001)$. Two studies $[50,51]$ investigated the effects of a self-monitoring app on fruit and vegetable intake and were able to identify increased mean vegetable intake from 4 to 5 servings per day ( $5 \pm 2$ mean servings/day, $p=0.024$; medium effect size, Cohen's $d=0.5$ ) [50] and two servings (95\% CI 0.1 to 3.8; $\mathrm{p}=0.02$, small effect size, Cohen's $d=0.18$ ) [51], 
respectively. One of these also showed a similar effect on fruit intake $(5 \pm 1$ mean servings/day, large effect size, Cohen's $d=1 ; p=0.023$ ) [50]. In both of these studies, app usage was regular (mean usage was $0.8 \pm 1.1$ times/day [51] and 72 dietary logs/driver over 20 weeks [50]). The impact of app usage on energy intake was statistically significant, with large effect sizes. These studies showed decreases in energy intake when participants used the app for self-monitoring, as compared to other technologies (such as a wearable bite counter) [54] or traditional [53] tools (such as paper journals).

Among studies that assessed $\mathrm{HbA} 1 \mathrm{c}$ as an outcome, two $[60,61]$ found significant improvement with large effect sizes $(-0.6 \pm 0.7 \%, \mathrm{p}<0.0001$, large effect size, Cohen's $\mathrm{d}=0.9$ and $-1.1 \% \pm 0.74$ vs control $+0.07 \% \pm 0.99$, large effect size, Cohen's $d=1.3, p<0.001$ respectively). One of these identified higher-frequency users (HFUs; at least once daily) to have higher decreases in HbA1c $(7.9 \pm 0.7 \%$ to $7.1 \pm 0.5 \%$, large effect size, Cohen's $d=1.3, p=0.0005)$ as compared to lower-frequency users (LFUs; less than once daily) [60], whereas the other found no relation between app usage and $\mathrm{HbA1c}[61]$.

\section{INTERVENTIONS WITH MULTIPLE ARMS}

Among studies using interventions with a multiple-arm design, one arm always consisted of accessibility to a mobile app as the sole intervention. Two studies $[57,46]$ found a significant decrease in BMI and/or weight for all groups from baseline with no significant differences across groups. One study [57], however, found the difference between app users and standard self-monitoring tools (calorie reference books, pedometers, and weight scales) to be clinically significant with a large effect size. Similarly, a study [49] found a significant decrease in BMI from baseline only in the app group. Significant decreases in weight among the app group as compared to a self-monitoring website but not to a paper-based diary were also detected. Four studies [43, 46, 49, 57] found the app effective as compared to baseline; however, they did not identify the clear superiority of the mobile app, as compared to other groups, across outcomes measured. 
Table 2: Key characteristics and nutrition outcomes of interventional studies included in the systematic review assessing the effectiveness of dietary mobile applications on nutritional outcomes in adults with chronic diseases

\begin{tabular}{|c|c|c|c|c|c|c|c|c|}
\hline \multirow{2}{*}{$\begin{array}{l}\text { Author, } \\
\text { year of } \\
\text { publicati } \\
\text { on } \\
\text { Country }\end{array}$} & \multirow{2}{*}{$\begin{array}{l}\text { Sample (n), Age } \\
\text { (yrs), BMI }{ }^{1} \\
(\mathrm{~kg} / \mathrm{m} 2), \text { other } \\
\text { inclusion } \\
\text { criteria }\end{array}$} & \multirow[t]{2}{*}{ Design } & \multirow[t]{2}{*}{ Methodology } & \multirow[t]{2}{*}{ Duration } & \multirow{2}{*}{$\begin{array}{l}\text { Chronic } \\
\text { Disease }\end{array}$} & \multirow{2}{*}{$\begin{array}{l}\text { Mobile app } \\
\text { Name }\end{array}$} & Results (Nutrition Outcomes) & \multirow{2}{*}{$\begin{array}{l}\text { Behavior } \\
\text { Theory }\end{array}$} \\
\hline & & & & & & & $\begin{array}{l}\text { App usage and/or effect of app usage on } \\
\text { outcomes }\end{array}$ & \\
\hline \multicolumn{9}{|c|}{ Mobile apps as the sole intervention } \\
\hline 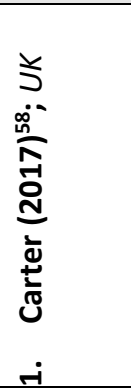 & $\begin{array}{l}\mathrm{n}=43 \\
\text { Mean age } \\
41 \pm 9 \mathrm{yrs} \\
\text { Mean } \mathrm{BMI} \\
34 \pm 4 \mathrm{~kg} / \mathrm{m}^{2}\end{array}$ & $\begin{array}{l}\text { Pilot RCT } \\
\text { (3-arms) } \\
\text { post-hoc } \\
\text { analysis } \\
\text { of app } \\
\text { arm }\end{array}$ & 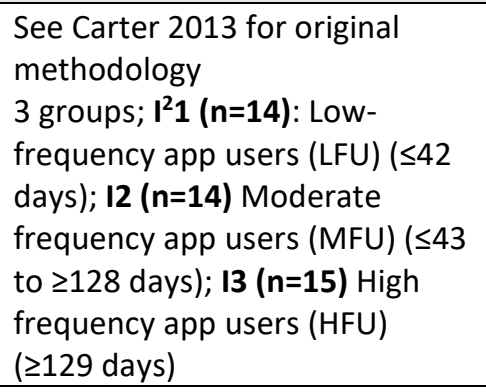 & $\begin{array}{l}6 \\
\text { months }\end{array}$ & Obesity & $\begin{array}{l}\mathrm{MMM}\left(\mathrm{SM}^{3}\right. \\
\text { app) }\end{array}$ & $\begin{array}{l}\mathrm{AM}^{4}: \downarrow \mathrm{Sig}^{5} \text {. in weight for HFU as } \\
\text { compared to MFU and LFU } \\
\text { (HFU }-4.7 \mathrm{Kg}(95 \% \mathrm{Cl}-8.2 \text { to }-1.1) \text { lower } \\
\text { weight than MFU \& }-6.4 \mathrm{~kg}(95 \% \mathrm{Cl}-10 \\
\text { to }-2.9) \text { lower weight than LFU, } \mathrm{p}<0.001)\end{array}$ & $\begin{array}{l}\text { Goal } \\
\text { setting, } \\
\text { Self- } \\
\text { monitor } \\
\text { ing \& } \\
\text { Feedbac } \\
\text { k }\end{array}$ \\
\hline \multirow{2}{*}{ 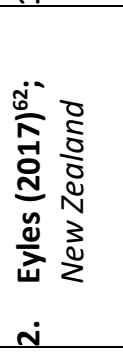 } & \multirow[t]{2}{*}{$\begin{array}{l}\mathrm{n}=66 ; \\
\text { Age }>40 \mathrm{yrs} ; \\
\text { history of CVD }\end{array}$} & \multirow[t]{2}{*}{$\mathrm{RCT}$} & \multirow{2}{*}{$\begin{array}{l}2 \text { groups; I ( } \mathbf{n = 3 3 )} \text { app: bar code } \\
\text { scanning enabled app that } \\
\text { suggests lower salt alternatives } \\
\& \text { guides patients on grocery } \\
\text { purchases } \\
\mathbf{C}^{6}(\mathbf{n}=\mathbf{3 3}) \text { Usual Care: access to } \\
\text { usual cardiac rehabilitation } \\
\text { services }\end{array}$} & \multirow[t]{2}{*}{1 month } & \multirow[t]{2}{*}{$\mathrm{CVD}^{7}$} & \multirow[t]{2}{*}{$\begin{array}{l}\text { SaltSwitch } \\
\text { (ED }{ }^{8} \text { app) }\end{array}$} & $\begin{array}{l}\mathrm{F} / \mathrm{N}-\mathrm{RH} \mathrm{H}^{9}: \downarrow \text { Sig. in salt purchases of I vs } \\
\mathrm{C}(-0.3 \mathrm{~g} / \mathrm{MJ}, 95 \% \mathrm{Cl},-0.58 \text { to }-0.03 \mathrm{~g} / \mathrm{MJ} \text {, } \\
\mathrm{p}=0.03 ; \text { medium effect size, Cohen's } \\
\mathrm{d}=0.55)\end{array}$ & \multirow[t]{2}{*}{$\begin{array}{l}\text { Not } \\
\text { reporte } \\
\text { d }\end{array}$} \\
\hline & & & & & & & App usage not reported & \\
\hline \multirow{2}{*}{ 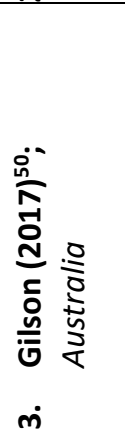 } & \multirow[t]{2}{*}{$\begin{array}{l}\mathrm{n}=19 \text { (Males) } \\
\text { Mean Age } \\
47.5 \pm 9.8 \mathrm{yrs} \\
\text { Mean } \mathrm{BMl} \\
31.2 \pm 4.6 \\
\mathrm{~kg} / \mathrm{m}^{2}\end{array}$} & \multirow[t]{2}{*}{$\begin{array}{l}\text { Prospecti } \\
\text { ve } \\
\text { intervent } \\
\text { ion } \\
\text { (single } \\
\text { arm) }\end{array}$} & \multirow[t]{2}{*}{$\begin{array}{l}1 \text { group }(\mathbf{n}=19) \text { app: truckdrivers } \\
\text { using self-monitoring } \\
\text { smartphone app and activity } \\
\text { tracker }\end{array}$} & \multirow[t]{2}{*}{$\begin{array}{l}5 \\
\text { months }\end{array}$} & \multirow[t]{2}{*}{ Obesity } & \multirow[t]{2}{*}{$\begin{array}{l}\text { Jawbone } \\
\text { UP (SM } \\
\text { app) }\end{array}$} & $\begin{array}{l}\mathrm{F} / \mathrm{N}-\mathrm{RH}: \uparrow \mathrm{Sig}^{10} \text { in mean fruit }(5 \pm 1 \\
\text { mean servings/day, large effect size, } \\
\text { Cohen's } d=1 ; p=0.023) \text { and mean } \\
\text { vegetable consumption ( } 5 \pm 2 \text { mean } \\
\text { servings/day, } p=0.024 ; \text { medium effect } \\
\text { size, Cohen's } d=0.5) \\
\text { No sig. diff. }{ }^{11} \text { in } \text { PA }^{12}\end{array}$ & \multirow[t]{2}{*}{$\begin{array}{l}\text { Not } \\
\text { reporte } \\
\text { d }\end{array}$} \\
\hline & & & & & & & $\begin{array}{l}\text { Median total dietary logs/driver was } 72 \\
\text { (IQR 115; range of } 0-466 \text { ) for the } 20 \\
\text { weeks }\end{array}$ & \\
\hline
\end{tabular}




\begin{tabular}{|c|c|c|c|c|c|c|c|c|}
\hline \multirow{2}{*}{ 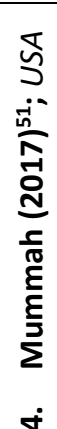 } & \multirow{2}{*}{$\begin{array}{l}\mathrm{n}=135 \\
\text { Age } 18-50 \mathrm{yrs} \\
\mathrm{BMI} 28-40 \\
\mathrm{~kg} / \mathrm{m}^{2} \text {, (part of } \\
\text { ongoing } \\
\text { weight loss } \\
\text { trial) }\end{array}$} & \multirow[t]{2}{*}{ RCT } & \multirow{2}{*}{$\begin{array}{l}2 \text { groups: I ( } n=68) \text { app focused } \\
\text { on self-monitoring of vegetables } \\
\text { intake \& } \\
\text { reminders/motivational } \\
\text { messaged to increase vegetable } \\
\text { consumption } \\
\text { C ( } n=67) \text { : Usual Care of parent } \\
\text { trial. } \\
\text { Parent Trial is weight loss trial of } \\
\text { either low-fat or low-carbs diet }\end{array}$} & \multirow[t]{2}{*}{$\begin{array}{l}2 \\
\text { months }\end{array}$} & \multirow[t]{2}{*}{ Obesity } & \multirow[t]{2}{*}{$\begin{array}{l}\text { Vegethon } \\
\text { (SM app) }\end{array}$} & $\begin{array}{l}\text { F/N-RH: } \uparrow \text { Sig in vegetable } \\
\text { consumption by I (adjusted mean } \\
\text { difference of } 2.0 \text { servings, } 95 \% \mathrm{Cl} 0.1 \text { to } \\
\begin{array}{l}3.8 ; p=0.02, \text { small effect size, Cohen's } \\
d=0.18 \text { ) }\end{array}\end{array}$ & \multirow{2}{*}{$\begin{array}{l}\text { SCT13 } \\
\& \\
\text { Several } \\
\text { constru } \\
\text { cts of } \\
\text { differen } \\
\mathrm{t} \\
\text { theories } \\
\text { used }\end{array}$} \\
\hline & & & & & & & $\begin{array}{l}\text { Self-monitoring logging: } 0.8 \pm 1.1 \\
\text { times/day }\end{array}$ & \\
\hline \multirow{2}{*}{ 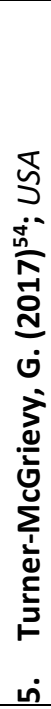 } & \multirow[t]{2}{*}{$\begin{array}{l}\mathrm{n}=81 \\
\text { Age } 18-65 \mathrm{yrs} \\
\text { BMI } 25- \\
49.9 \mathrm{~kg} / \mathrm{m}^{2}\end{array}$} & \multirow[t]{2}{*}{ RCT } & \multirow[t]{2}{*}{$\begin{array}{l}2 \text { groups: I1 ( } \mathbf{n}=\mathbf{4 2} \text { ) app } \\
\text { participants used to the app to } \\
\text { self-monitor food intake, PA and } \\
\text { weight goals (based on energy } \\
\text { needs) \& twice weekly podcasts } \\
\mathbf{I 2} \text { ( } \mathrm{n}=\mathbf{3 9} \text { ) Bite Counter } \\
\text { participants used a bite counting } \\
\text { device (wearable on the wrist) } \\
\text { with daily "bite" goals (based on } \\
\text { energy needs) \& twice weekly } \\
\text { podcasts }\end{array}$} & \multirow[t]{2}{*}{$\begin{array}{l}6 \\
\text { months }\end{array}$} & \multirow[t]{2}{*}{ Obesity } & \multirow[t]{2}{*}{$\begin{array}{l}\text { Fatsecret's } \\
\text { (SM app) }\end{array}$} & $\begin{array}{l}\text { F/N-RH: } \downarrow \text { sig. in energy intake from } \\
\text { baseline in both groups } \\
\text { (I1 }-620 \pm 157.3 \mathrm{Kcal} / \mathrm{d} \text { vs } \\
\mathbf{I} 2-456.2 \pm 166.9 \mathrm{Kcal} / \mathrm{d}, \mathrm{p}<0.001) \text {. } \\
\text { No sig diff between groups (Large effect } \\
\text { size, Cohen's } \mathrm{d}=1.01 \text { ) } \\
\text { AM: } \downarrow \text { sig. in weight from baseline in } \\
\text { both groups (I1 }-6.8 \pm 0.8 \mathrm{Kg} \text { vs } \mathbf{I 2} \text { - } \\
3.0 \pm 0.8 \mathrm{Kg}, \mathrm{p}<0.001 \text { ) } \\
\downarrow \text { sig. in weight of app group (I1) vs } \mathbf{1 2} \text {, } \\
\mathrm{p}=0.001 \text {, large effect size, Cohen's } \\
d=4.75\end{array}$ & \multirow[t]{2}{*}{ SCT } \\
\hline & & & & & & & $\begin{array}{l}\text { Higher number of diet tracking days was } \\
\text { significantly correlated with weight loss } \\
(\mathrm{r}=-0.33, \mathrm{P}<0.01)\end{array}$ & \\
\hline \multirow{2}{*}{ 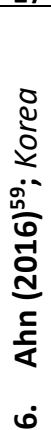 } & \multirow[t]{2}{*}{$\begin{array}{l}\mathrm{n}=26 \\
\text { Mean age: } \\
\text { I: } 50.5 \mathrm{yrs} \& \mathrm{C} \text { : } \\
49.7 \mathrm{yrs}\end{array}$} & \multirow[t]{2}{*}{$\begin{array}{l}\text { Non- } \\
\text { equivale } \\
\mathrm{nt} \\
\text { control } \\
\text { group } \\
\text { (conveni } \\
\text { ent } \\
\text { sample } \\
\text { allocatio } \\
\text { n) }\end{array}$} & \multirow[t]{2}{*}{ 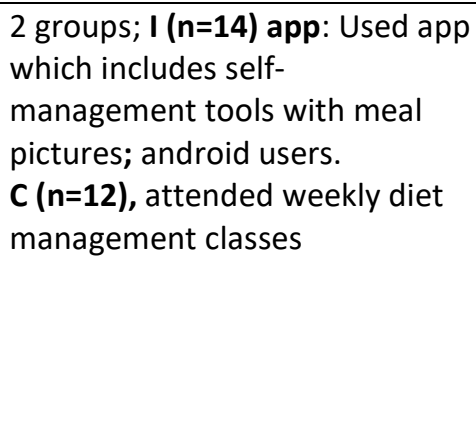 } & \multirow[t]{2}{*}{1 month } & \multirow[t]{2}{*}{ DM } & \multirow[t]{2}{*}{$\begin{array}{l}\text { DMDMG }^{14} \\
\text { (SM app) }\end{array}$} & $\begin{array}{l}\text { F/N-RH: I group had Sig } \uparrow \text { vs } C \text { in: } \\
\text { dietary attitudes score; dietary behavior } \\
\text { score }(p<0.01) \text {, small effect size, } \\
\text { Cohen's } d=0.25 \\
\text { No sig diff in nutrient intakes; \& } \\
\text { nutrition knowledge } \\
\text { AM: No sig diff in weight \& BMI }\end{array}$ & \multirow[t]{2}{*}{$\begin{array}{l}\text { Not } \\
\text { Reporte } \\
\text { d }\end{array}$} \\
\hline & & & & & & & App usage not reported & \\
\hline
\end{tabular}




\begin{tabular}{|c|c|c|c|c|c|c|c|c|}
\hline \multirow{2}{*}{ 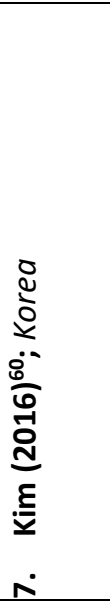 } & \multirow[t]{2}{*}{$\begin{array}{l}\mathrm{n}=29 ; \\
\text { Age 20-80yrs } \\
\text { Hba1c } \geq 6.5\end{array}$} & \multirow[t]{2}{*}{$\begin{array}{l}\text { Pilot } \\
\text { study } \\
\text { (single } \\
\text { arm) }\end{array}$} & \multirow{2}{*}{$\begin{array}{l}1 \text { group; I ( } \mathbf{n}=\mathbf{2 9} \text { ): app focused } \\
\text { on dietary self-monitoring \& } \\
\text { glucose self-monitoring } \\
\text { (glucometer connected by } \\
\text { Bluetooth) \& PA tracking } \\
\text { (tracker \& educational videos). } \\
\text { Alarm messages when BG was } \\
\text { high or low \& family member } \\
\text { contact in extreme BG } \\
\text { deviations. Physician access to } \\
\text { patient data through website }\end{array}$} & \multirow[t]{2}{*}{$\begin{array}{l}3 \\
\text { months }\end{array}$} & \multirow[t]{2}{*}{ T2DM } & \multirow[t]{2}{*}{$\begin{array}{l}\text { PSDCS }^{15} \\
\text { (SM \& ED } \\
\text { app) }\end{array}$} & $\begin{array}{l}\text { F/N-RH: } \uparrow \text { Sig in "general diet" and } \\
\text { "exercise" scores of summary of DM } \\
\text { self-care activities (SDSCA), p<0.001 } \\
\text { AM: No Sig. change in weight } \\
\text { BD/MT \& } \mathrm{P}^{16}: \downarrow \text { Sig. in Hba1c from } \\
\text { baseline } \\
(-0.6 \pm 0.7 \%, p<0.0001 \text {, large effect size } \\
\text { Cohen's } d=0.9)\end{array}$ & \multirow[t]{2}{*}{$\begin{array}{l}\text { Not } \\
\text { Reporte } \\
\text { d }\end{array}$} \\
\hline & & & & & & & $\begin{array}{l}\text { Categorized by glucometer input } \\
\text { frequency at least once daily vs less } \\
\text { than once daily: Hba } 1 c \text { decreased sig } \\
\text { among higher frequency users } \\
(7.9 \pm 0.7 \% \text { to } 7.1 \pm 0.5 \% \text {, large effect size, } \\
\text { Cohen's } d=1.3, p=0.0005)\end{array}$ & \\
\hline 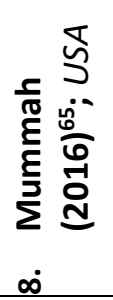 & $\begin{array}{l}\mathrm{n}=17 \\
\text { Age } 18-50 \mathrm{yrs} \\
\text { BMI } 28-40 \\
\mathrm{~kg} / \mathrm{m} 2 \text {, (part } \\
\text { of ongoing } \\
\text { weight loss } \\
\text { trial) }\end{array}$ & $\mathrm{RCT}$ & $\begin{array}{l}2 \text { groups: I }(n=8) \text { app focused on } \\
\text { self-monitoring of vegetables } \\
\text { intake } \\
C(n=9) \text { : Usual Care of parent } \\
\text { trial. } \\
\text { Parent Trial is weight loss trial of } \\
\text { either low-fat or low-carbs diet }\end{array}$ & $\begin{array}{l}3 \\
\text { months }\end{array}$ & Obesity & $\begin{array}{l}\text { Vegethon } \\
\text { (SM app) }\end{array}$ & $\begin{array}{l}\text { F/N-RH: } \uparrow \text { Sig in vegetable } \\
\text { consumption by I (adjusted mean } \\
\text { difference of } 7.4 \text { servings, } p=0.02 \text { ), } \\
\uparrow \text { Sig in green leafy, cruciferous and } \\
\text { dark yellow vegetables (mean } \\
\text { difference of } 2.6,1.6 \& 0.8 \text { servings, } \\
p=0.04, p=0.04, p=0.004 \text { respectively) }\end{array}$ & $\begin{array}{l}\text { Several } \\
\text { constru } \\
\text { cts of } \\
\text { differen } \\
\mathrm{t} \\
\text { theories } \\
\text { used }\end{array}$ \\
\hline 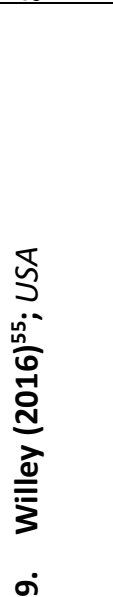 & $\begin{array}{l}\mathrm{n}=10 ; \\
\text { Age 30-50yrs } \\
\text { BMI 27-36 } \\
\mathrm{kg} / \mathrm{m}^{2}\end{array}$ & $\begin{array}{l}\text { Quasi- } \\
\text { experime } \\
\text { ntal pilot } \\
\text { (single- } \\
\text { arm) }\end{array}$ & $\begin{array}{l}1 \text { group: app focused on in-app } \\
\text { nutrition education, recipes, } \\
\text { automated feedback based on } \\
\text { in-app questions (daily). PA } \\
\text { program through in-app } \\
\text { personal training instructions \& } \\
\text { video }\end{array}$ & $\begin{array}{l}3 \\
\text { months }\end{array}$ & Obesity & $\begin{array}{l}\text { YouPlus } \\
\text { Health (ED } \\
\text { app) }\end{array}$ & $\begin{array}{l}\text { AM: } \downarrow \text { sig. in weight } \\
(-6.12 \mathrm{~kg}, \mathrm{z}=-2.805, \text { medium effect size } \\
\text { Cohen's } d=0.63, p=0.005) \\
\downarrow \text { sig. in WC } \mathrm{C}^{17} \\
(-7.2 \mathrm{~cm}, \mathrm{z}=-2.825, \text { medium effect size } \\
\text { Cohen's } d=0.63, p=0.005) \\
\text { BD/MT \&P: } \downarrow \text { Sig.in SBP } 18(-18.6 \mathrm{mmHg} \text {, } \\
\mathrm{z}=-2.810, \text { effect size }=-0.63, p=0.05) \& \\
\text { DBP }{ }^{19}(-6.4 \mathrm{mmHg}, \mathrm{z}=-2.805, \text { medium } \\
\text { effect size Cohen's } d=-0.63, p=0.05) \\
\uparrow \text { Sig. in HDL (+4mg/dl, } \mathrm{z}=2.044, \\
\text { medium effect size Cohen's } d=-0.43 p= \\
0.04)\end{array}$ & $\begin{array}{l}\text { Not } \\
\text { reporte } \\
\text { d }\end{array}$ \\
\hline
\end{tabular}




\begin{tabular}{|c|c|c|c|c|c|c|c|c|}
\hline & & & & & & & $\begin{array}{l}\text { Rho correlation coefficients calculated } \\
\text { between app usage and all variables. } \\
\text { More app use was correlated with } \\
\text { greater improvements, but no statistical } \\
\text { significance detected (rho varied from } \\
0.109 \text { to } 0.442 \text { ) }\end{array}$ & \\
\hline \multirow[b]{2}{*}{ 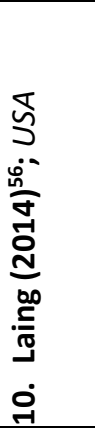 } & \multirow[t]{2}{*}{$\begin{array}{l}\mathrm{n}=212 ; \\
\text { Age } \geq 18 \mathrm{yrs} \\
\mathrm{BMI} \geq 25 \mathrm{~kg} / \mathrm{m}^{2}\end{array}$} & \multirow[t]{2}{*}{$\mathrm{RCT}$} & \multirow{2}{*}{$\begin{array}{l}2 \text { groups; I ( } n=105) \text { app \& usual } \\
\text { primary care. App focused on } \\
\text { dietary \& PA self-monitoring; } \\
\text { C ( } n=107) \text { : usual primary care } \\
\text { only. } \\
\text { All participants received an } \\
\text { educational one-page handout } \\
\text { on healthy eating from } \\
\text { www.myplate.gov }\end{array}$} & \multirow[t]{2}{*}{$\begin{array}{l}6 \\
\text { months }\end{array}$} & \multirow[t]{2}{*}{ Obesity } & \multirow[t]{2}{*}{$\begin{array}{l}\text { MyFitnessP } \\
\text { al (SM app) }\end{array}$} & $\begin{array}{l}\text { AM: No Sig. change in weight }(-0.27 \mathrm{Kg} \\
95 \% \mathrm{Cl},-1.13 \text { to } 0.6, p=0.53) \\
\text { BD/MT \&P: No Sig. change in SBP }\end{array}$ & \multirow{2}{*}{$\begin{array}{l}\text { Element } \\
\text { S of SCT } \\
\text { (self- } \\
\text { monitor } \\
\text { ing, } \\
\text { goal- } \\
\text { setting } \\
\& \\
\text { Feedbac } \\
\text { k) }\end{array}$} \\
\hline & & & & & & & $\begin{array}{l}\text { Log in to the app decrease between } \\
\text { beginning and end of intervention: } \\
\text { Mean log-in (number of times) was 1st } \\
\text { month: } 20.9 \text { and 6th month: } 6.2\end{array}$ & \\
\hline \multirow{2}{*}{ 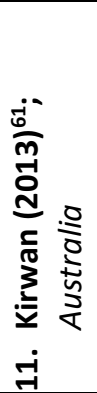 } & \multirow[t]{2}{*}{$\begin{array}{l}\mathrm{n}=72 ; \\
\text { Age 18-65yrs } \\
\text { Hba1c } \geq 7.5\end{array}$} & \multirow[t]{2}{*}{ RCT } & \multirow[t]{2}{*}{$\begin{array}{l}2 \text { groups: I ( } n=36) \text { : app \& usual } \\
\text { care \& personalized text } \\
\text { messages. App focused on } \\
\text { diabetes self-management tools } \\
\text { (BG monitoring, insulin \& } \\
\text { medication dosage, diet \& PA) } \\
\text { C ( } n=36) \text { : Usual Care }\end{array}$} & \multirow[t]{2}{*}{$\begin{array}{l}9 \\
\text { months }\end{array}$} & \multirow[t]{2}{*}{ T1DM } & \multirow[t]{2}{*}{$\begin{array}{l}\text { Glucose } \\
\text { Buddy (SM } \\
\text { app) }\end{array}$} & $\begin{array}{l}\text { F/N-RH: } \uparrow \text { Sig in "general diet" scores } \\
\text { of Summary of DM self-care activities } \\
\text { (SDSCA) from baseline }(F=5.30 ; p<0.05) \\
\text { BD/MT \&P: } \downarrow \text { Sig. in Hba1c from } \\
\text { baseline \& between groups (I: - } \\
1.1 \% \pm 0.74 \text { vs C: }+0.07 \% \pm 0.99 \text {, Large } \\
\text { Effect size, Cohen's } d=1.3, p<0.001)\end{array}$ & \multirow[t]{2}{*}{$\begin{array}{l}\text { Not } \\
\text { Reporte } \\
\text { d }\end{array}$} \\
\hline & & & & & & & $\begin{array}{l}\text { No sig diff between engagement with } \\
\text { app and change in } \mathrm{Hbac1}\end{array}$ & \\
\hline
\end{tabular}




\begin{tabular}{|c|c|c|c|c|c|c|c|c|}
\hline & $\begin{array}{l}\mathrm{n}=96 ; \\
\text { Age 18-60yrs } \\
\text { BMI 25- } \\
45 \mathrm{~kg} / \mathrm{m}^{2}\end{array}$ & \begin{tabular}{|l|} 
RCT \\
(Post-hoc \\
analysis)
\end{tabular} & $\begin{array}{l}\text { See Turner-McGrievy, } 2011^{14} \text { for } \\
\text { original methodology. Re- } \\
\text { clustered by self-monitoring } \\
\text { method } \\
\text { I1: PA self-monitoring group (85, } \\
48 \text { PA app users, } 37 \text { non-PA app } \\
\text { users) } \\
\text { 12 Diet self-monitoring group } \\
\text { (78, } 37 \text { mobile app, } 17 \text { paper } \\
\text { journals, } 24 \text { website) }\end{array}$ & \begin{tabular}{|l|}
6 \\
months
\end{tabular} & Obesity & $\begin{array}{l}\text { Fatsecret's } \\
\text { (SM app) } \\
\text { other apps } \\
\text { of } \\
\text { preference } \\
\text { allowed } \\
\text { (RunKeepe } \\
\text { r, } \\
\text { MyFitnessP } \\
\text { al, Loseit!) }\end{array}$ & $\begin{array}{l}\text { F/N-RH: } \downarrow \text { sig. energy intake by app } \\
\text { users }(1437 \pm 188 \mathrm{Kcal} / \mathrm{d} \text { app vs } \\
2049 \pm 175 \mathrm{Kcal} / \mathrm{d} \text { Paper Journal; } \mathrm{p}=0.01 \text {, } \\
\text { large effect size, } \\
\text { Cohen's } \mathrm{d}=-3.4) \\
\text { No sig diff in fat, added sugar, fruit \& } \\
\text { vegetable intake } \\
\\
\text { AM: } \downarrow \text { sig. in BMl of PA app users } \\
\left(31.5 \pm 0.5 \mathrm{~kg} / \mathrm{m}^{2} \text { vs non-PA app users }\right. \\
32.5 \pm 0.5 \mathrm{~kg} / \mathrm{m}^{2} ; p=0.02, \text { large effect } \\
\text { size, Cohen's d= -2) } \\
\downarrow \text { sig. in Weight loss by PA app users } \\
(-3.7 \pm 1.5 \% \text { vs non-PA app users } \\
-0.5 \pm 1.5 \% ; p=0.01, \text { large effect size, } \\
\text { Cohen's } d=-2.1)\end{array}$ & $\begin{array}{l}\text { Social } \\
\text { cognitiv } \\
\text { e } \\
\text { Theory } \\
\text { (podcas } \\
\text { ts only) }\end{array}$ \\
\hline 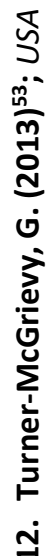 & & & & & & & & \\
\hline
\end{tabular}




\begin{tabular}{|c|c|c|c|c|c|c|c|c|}
\hline \multicolumn{9}{|c|}{ Comprehensive Interventions (multiple arms) with one arm mobile app as the sole intervention } \\
\hline 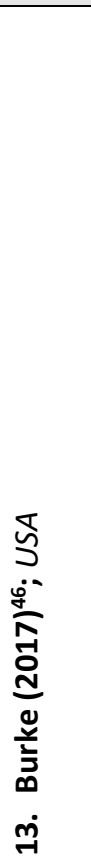 & $\begin{array}{l}\mathrm{n}=39 \\
\text { Age } \geq 18 \mathrm{yrs} \\
\mathrm{BMI} \geq 27 \text { and } \\
\leq 43 \mathrm{~kg} / \mathrm{m}^{2}\end{array}$ & $\begin{array}{l}\text { RCT (3 } \\
\text { arms) }\end{array}$ & $\begin{array}{l}3 \text { groups: I1 ( } \mathbf{n}=\mathbf{1 3} \text { ): app } \\
\text { I2 ( } \mathbf{n}=\mathbf{1 3}) \text { : app + FB (daily } \\
\text { feedback messages provided by } \\
\text { a software integrated with the } \\
\text { app) } \\
\text { I3 ( } \mathbf{n}=\mathbf{1 3}) \text { : app +FB + group } \\
\text { sessions (3 group behavioral } \\
\text { weight loss sessions) }\end{array}$ & $\begin{array}{l}3 \\
\text { months }\end{array}$ & Obesity & $\begin{array}{l}\text { Loseit! (SM } \\
\text { app) }\end{array}$ & 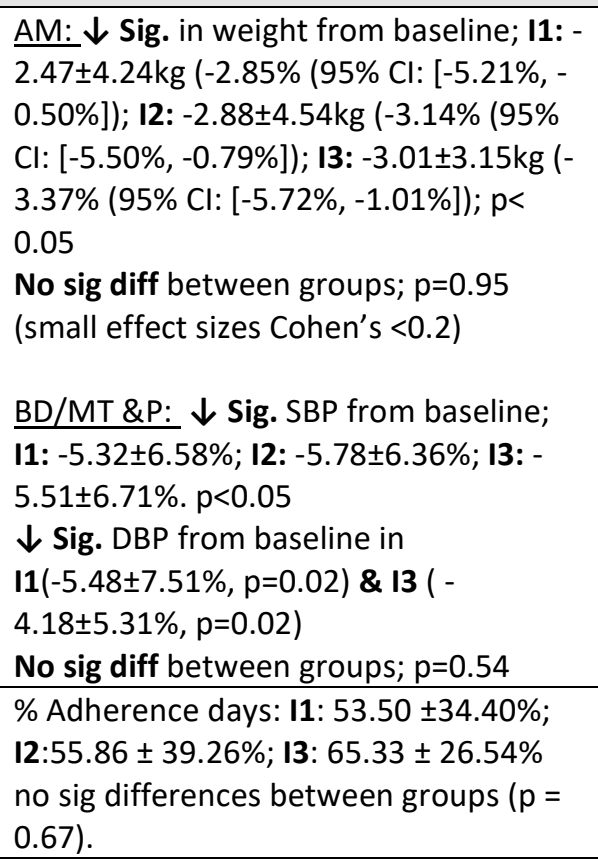 & $\begin{array}{l}\text { Not } \\
\text { reporte } \\
\text { d }\end{array}$ \\
\hline 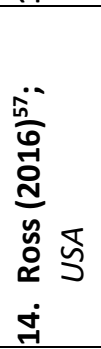 & $\begin{array}{l}\mathrm{n}=80 ; \\
\text { Age } 18-70 \text { yrs } \\
\text { BMI 27-40 } \\
\mathrm{kg} / \mathrm{m}^{2}\end{array}$ & $\begin{array}{l}\text { RCT (3 } \\
\text { arms) }\end{array}$ & $\begin{array}{l}3 \text { groups: } \mathbf{I 1}(\mathbf{n}=\mathbf{2 6}) \text { Standard } \\
\text { self-monitoring tools (ST), } \\
\text { provided with a calorie } \\
\text { reference book, a pedometer \& } \\
\text { body weight scale } \\
\mathbf{I 2}(\mathbf{n}=\mathbf{2 7}) \text { app: Technology based } \\
\text { self-monitoring tools, use of }\end{array}$ & $\begin{array}{l}6 \\
\text { months }\end{array}$ & Obesity & $\begin{array}{l}\text { Fitbit app } \\
\text { (SM app) } \\
\text { \& Fitbit } \\
\text { tracker }\end{array}$ & $\begin{array}{l}\text { AM: } \downarrow \text { Sig. in weight from baseline; } \\
\text { (I1: }-1.28 \pm 1.19 \mathrm{~kg} ; \text { I2: }-4,04 \pm 1.37 \mathrm{~kg} ; \mathrm{I3}:- \\
6.40 \pm 1.17 \mathrm{~kg}, \mathrm{p}=0.042) \\
\text { No sig diff between groups, clinically sig } \\
\text { diff between } 13 \text { \& the other groups } \\
\text { (Large effect sizes, Cohen's } d=-4.3 \text { and } \\
\text { Cohen's d= }-1.8 \text { for } I 1 \& 13 \text { and } 12 \& 13 \\
\text { respectively) }\end{array}$ & $\begin{array}{l}\text { Goal } \\
\text { setting, } \\
\text { feedbac } \\
\mathrm{k} \& \\
\text { Reinforc } \\
\text { ement } \\
\text { and } \\
\text { Self- }\end{array}$ \\
\hline
\end{tabular}




\begin{tabular}{|c|c|c|c|c|c|c|c|c|}
\hline & & & $\begin{array}{l}\text { Fitbit \& Fitbit app along with } \\
\text { Fitbit scale } \\
\text { I3 ( } \mathbf{n = 2 7 )} \text { app + PHONE } \\
\text { Technology based tools with } \\
\text { phone-based intervention. Same } \\
\text { as app with } 14 \text { structured phone } \\
\text { calls gradually descending in } \\
\text { frequency. }\end{array}$ & & & & $\begin{array}{l}\text { App usage sig higher by } \mathbf{I} \mathbf{3} \text { vs } \mathbf{I 2} \text {; mean } \\
\text { days of caloric intake monitoring of } \mathbf{I} 2 \text { : } \\
83.7 \pm 11.12 \text { vs } \mathbf{1 3} 126.59 \pm 11.11, p=0.009\end{array}$ & $\begin{array}{l}\text { monitor } \\
\text { ing }\end{array}$ \\
\hline \multirow[b]{2}{*}{ 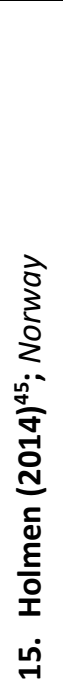 } & \multirow[t]{2}{*}{$\begin{array}{l}\mathrm{n}=151 \\
\text { Age } \geq 18 \text { yrs; } \\
\text { Hba } 1 \mathrm{c} \geq 7.1 \%\end{array}$} & \multirow[t]{2}{*}{$\begin{array}{l}\text { RCT (3 } \\
\text { arms) }\end{array}$} & \multirow{2}{*}{$\begin{array}{l}3 \text { groups; I1 ( } \mathbf{n}=\mathbf{5 1 ) : ~ a p p ~} \\
\text { focusing on dietary, physical } \\
\text { activity (PA) \& glucose self- } \\
\text { monitoring tools } \\
\text { I2 ( } \mathrm{n}=50 \text { ): app \& Health } \\
\text { Counseling (HC). I1 plus phone- } \\
\text { based health counseling from } \\
\text { nurse specialist } \\
\text { I3 }(n=50) \text { : Control Group: Usual } \\
\text { care }\end{array}$} & \multirow[t]{2}{*}{1 year } & \multirow[t]{2}{*}{ T2DM } & \multirow[t]{2}{*}{$\begin{array}{l}\text { Few Touch } \\
\text { App (SM } \\
\text { app) }\end{array}$} & $\begin{array}{l}\text { F/N-RH: No Sig. diff in PA or eating } \\
\text { habits } \\
\text { AM: No Sig. change in weight in all } 3 \\
\text { groups } \\
\text { BD/MT \&P: No Sig diff in Hba1c } \\
\text { between groups }\end{array}$ & \multirow{2}{*}{$\begin{array}{l}\text { Transth } \\
\text { eoretica } \\
\text { I model, } \\
\text { proble } \\
\mathrm{m} \\
\text { solving, } \\
\text { and } \\
\mathrm{Ml}^{20} \\
\text { (used in } \\
\text { counseli } \\
\mathrm{ng} \text { ) }\end{array}$} \\
\hline & & & & & & & $\begin{array}{l}\text { Categorized as substantial ( } \geq 50 \\
\text { interactions with app over } 6 \text { months) vs } \\
\text { not-substantial users } \\
\text { I1: } 39 \% \text { substantial users; } \mathbf{I 2}: 34 \% \\
\text { substantial users; No sig diff across } \\
\text { groups between substantial and not } \\
\text { substantial users. } \\
\text { Participants over } 63 y \text { rs were more likely } \\
\text { to be substantial users (OR } 2.7 ; 95 \% \mathrm{Cl} \\
1.02-7.12 ; p=0.045 \text { ) }\end{array}$ & \\
\hline
\end{tabular}




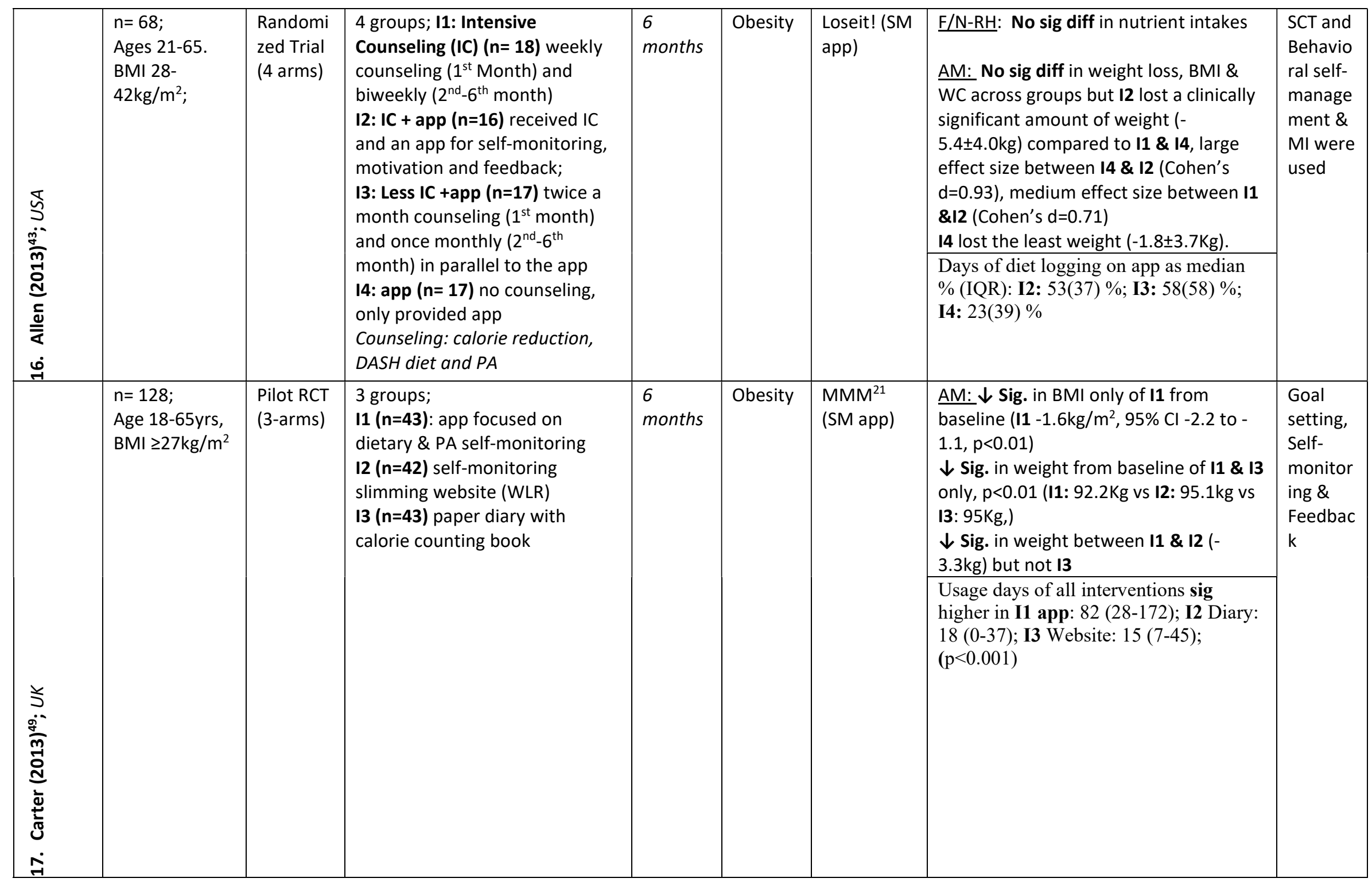




\begin{tabular}{|c|c|c|c|c|c|c|c|c|}
\hline \multicolumn{9}{|c|}{ Counseling Interventions supported by app } \\
\hline \multirow[b]{2}{*}{ 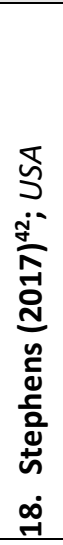 } & \multirow[t]{2}{*}{$\begin{array}{l}\mathrm{n}=62 ; \\
\text { Age 18-25yrs } \\
\text { BMI 25- } \\
45 \mathrm{~kg} / \mathrm{m}^{2}\end{array}$} & \multirow[t]{2}{*}{$\mathrm{RCT}$} & \multirow{2}{*}{$\begin{array}{l}2 \text { groups: I ( } \mathbf{n = 3 1 )} \text { app + Health } \\
\text { Coach. } 2 \text { counselling sessions at } \\
\text { baseline (diet \& PA); } \\
\text { motivational text messages by } \\
\text { health coach \& mobile app. } \\
\text { App focused on dietary \& PA } \\
\text { self-monitoring. } \\
\mathbf{I 2} \text { ( } \mathbf{n}=\mathbf{3 1} \text { ) Waiting control (asked } \\
\text { not to use a smartphone app } \\
\text { during the intervention) }\end{array}$} & \multirow[t]{2}{*}{$\begin{array}{l}3 \\
\text { months }\end{array}$} & \multirow[t]{2}{*}{ Obesity } & \multirow[t]{2}{*}{$\begin{array}{l}\text { Loseit! (SM } \\
\text { app) }\end{array}$} & $\begin{array}{l}\text { F/N-RH: } \uparrow \text { Sig in Fiber consumption (I } \\
18.4 \mathrm{~g} \text { vs } C 13.0 \mathrm{~g}, \mathrm{p}=0.049) \\
\mathrm{AM}: \downarrow \text { sig. in weight by I }(-1.8 \mathrm{Kg} \text { vs } \\
+0.3 \mathrm{Kg} \text { by C; } p<0.01) \\
\downarrow \text { sig. } \mathrm{BMI} \& \mathrm{WC} \text { by I }(\mathrm{p}<0.01)\end{array}$ & \multirow[t]{2}{*}{$\begin{array}{l}\text { Self- } \\
\text { efficacy } \\
\text { theory } \\
\text { (constru } \\
\text { ct of } \\
\text { SCT) }\end{array}$} \\
\hline & & & & & & & $\begin{array}{l}62 \% \text { of participants logged diet on more } \\
\text { than } 50 \% \text { of the days (logging frequency } \\
\text { was not associated with weight loss, } \\
p=0.226) \\
21 \% \text { of participants logged PA on more } \\
\text { than } 50 \% \text { of the days }(0.03 \mathrm{Kg} \text { weight } \\
\text { loss per additional day of PA logged, } \\
\mathrm{p}=0.026)\end{array}$ & \\
\hline \multirow{2}{*}{ 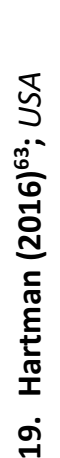 } & \multirow{2}{*}{$\begin{array}{l}\mathrm{n}=54 \\
\text { (Females) } \\
\text { Age } 40-75 y r s \\
\mathrm{BMI} \geq 27.5 \\
\mathrm{~kg} / \mathrm{m}^{2} ; \text { with } \\
\text { elevated } \\
\text { Breast Cancer } \\
\text { risk (Gail } \\
\text { model score } \geq \\
1.7 \text { ) }\end{array}$} & \multirow[t]{2}{*}{$\mathrm{RCT}$} & \multirow{2}{*}{$\begin{array}{l}2 \text { groups; I ( } n=36) \text { : app focused } \\
\text { on dietary \& PA self-monitoring, } \\
\text { plus PA tracker \& phone } \\
\text { counselling ( } 2 \text { monthly phone } \\
\text { calls-30min each) } \\
\text { C ( } n=18) \text { : Usual Care. Provided } \\
\text { with a paper copy of USDA } \\
\text { Dietary Guidelines \& } 2 \text { phone } \\
\text { calls (15 min each) without } \\
\text { counseling }\end{array}$} & \multirow[t]{2}{*}{6} & \multirow[t]{2}{*}{$\begin{array}{l}\text { Obesity } \\
\& \\
\text { Breast } \\
\text { Cancer } \\
\text { Risk }\end{array}$} & \multirow[t]{2}{*}{$\begin{array}{l}\text { MyFitnessP } \\
\text { al (SM app) } \\
\text { \& Fitbit } \\
\text { tracker }\end{array}$} & $\begin{array}{l}\text { F/N-RH: } \uparrow \text { Sig in PA } \\
(I+15.01 \pm 14.2 \text { min } / d \text { vs } C+10.9 \pm 10.1 \\
\min / d, p=0.01, \text { small effect size, Cohen's } \\
d=0.33) \\
\frac{\text { AM: } \downarrow \text { Sig. in weight }}{(I-4.4 \mathrm{~kg} \pm 4.3 \text { vs } C-0.8 \pm 3.8 \mathrm{~kg}, p=0.004,} \\
\text { Large effect size, Cohen's } d=0.88)\end{array}$ & \multirow[t]{2}{*}{$\begin{array}{l}\text { SCT } \\
\text { used } \\
\text { during } \\
\text { counsell } \\
\text { ing }\end{array}$} \\
\hline & & & & & & & $\begin{array}{l}\text { Not reported (only Fitbit wearable } \\
\text { device reported) }\end{array}$ & \\
\hline
\end{tabular}




\begin{tabular}{|c|c|c|c|c|c|c|c|c|}
\hline \multirow[t]{2}{*}{ 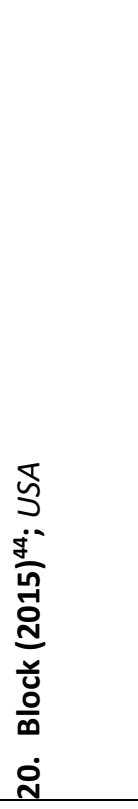 } & \multirow[t]{2}{*}{$\begin{array}{l}\mathrm{n}=339 \\
\text { Ages } 30-69 ; \\
\text { BMl>25 kg/m², } \\
\text { pre-diabetics. }\end{array}$} & \multirow[t]{2}{*}{$\begin{array}{l}\text { RCT } \\
\text { (waiting } \\
\text { controls) }\end{array}$} & \multirow[t]{2}{*}{$\begin{array}{l}2 \text { groups; I: app ( } \mathbf{n}=163 \text { ) weekly } \\
\text { contact by automated emails, } \\
\text { mobile phone reminders, } \\
\text { interactive voice response } \\
\text { phone calls and supportive } \\
\text { mobile app } \\
\text { app focused on dietary \& PA } \\
\text { self-monitoring } \\
\text { C: Waiting controls ( } \mathbf{n = 1 7 6 )} \\
\text { usual care at the clinic }\end{array}$} & \multirow[t]{2}{*}{$\begin{array}{l}6 \\
\text { months }\end{array}$} & \multirow[t]{2}{*}{ Pre-DM } & \multirow[t]{2}{*}{$\begin{array}{l}\text { Alive-PD } \\
\text { (SM app) }\end{array}$} & $\begin{array}{l}\text { AM: I group had Sig } \downarrow \text { vs C in } \\
\text { BMI (change }(95 \% \mathrm{Cl}) \text { of }-1.05(-1.06,- \\
1.05) \text { vs } 0.39(-0.39,-0.38) \mathrm{kg} / \mathrm{m}^{2} \text { of I \& } \\
\text { C respectively } \mathrm{p}<0.001) \text {; } \\
\text { Weight: (change }(95 \% \mathrm{Cl})-3.26(-3.26,- \\
\text { 3.25) vs }-1.26 \mathrm{Kg}(-1.27,-1.26), \text { of I \& C } \\
\text { respectively, } \mathrm{p}<0.001) ; \\
\text { WC (change }(95 \% \mathrm{Cl})-4.56(-4.69,-4.43) \\
\text { vs }-2.22 \mathrm{~cm}(-2.36,-2.09), \text { of I \& C } \\
\text { respectively, p<0.001) } \\
\text { BD/MT \&P: I group had Sig } \downarrow \text { vs C in } \\
\text { Hba1c (change }(95 \% \mathrm{Cl})-0.26(-0.27,- \\
0.24) \text { vs -0.18 }(-0.19,-0.16) \% \text { of I \& C } \\
\text { respectively, p<0.001); } \\
\text { Fasting glucose (change }(95 \% \mathrm{Cl})-7.36(- \\
7.85,-6.87) \text { vs }-2.19(-2.64,-1.73) \mathrm{mg} / \mathrm{dl} \\
\text { of I \& C respectively, } \mathrm{p}<0.001) \\
\text { App usage not reported }\end{array}$ & \multirow[t]{2}{*}{$\begin{array}{l}\text { Goal } \\
\text { setting, } \\
\text { constru } \\
\text { cts from } \\
\text { SCT, } \\
\text { TPB }^{22} \text {, } \\
\text { behavio } \\
\text { ral } \\
\text { econom } \\
\text { ics and } \\
\text { positive } \\
\text { psychol } \\
\text { ogy }\end{array}$} \\
\hline & & & & & & & & \\
\hline \multirow[t]{2}{*}{ 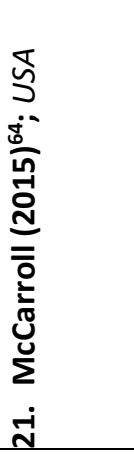 } & \multirow[t]{2}{*}{$\begin{array}{l}\mathrm{n}=50 \text { women } \\
\text { history of } \\
\text { Stage I or II } \\
\text { endometrial } \\
\text { cancer (EC) } \\
\text { and/or breast } \\
\text { cancer (BC); } \\
\text { Age 18-75 yrs }\end{array}$} & \multirow[t]{2}{*}{$\begin{array}{l}\text { Prospecti } \\
\text { ve } \\
\text { intervent } \\
\text { ion } \\
\text { (single } \\
\text { arm) }\end{array}$} & \multirow[t]{2}{*}{$\begin{array}{l}1 \text { group: I ( } \mathbf{n}=50 \text { ) } \\
\text { Comprehensive lifestyle } \\
\text { program (exercise \& nutrition } \\
\text { counselling) provided by } \\
\text { multidisciplinary team with real- } \\
\text { time feedback \& usage of app } \\
\text { app focused on dietary \& PA } \\
\text { self-monitoring }\end{array}$} & \multirow[t]{2}{*}{1 month } & \multirow[t]{2}{*}{$\begin{array}{l}\text { Obesity } \\
\text { EC } \\
B C\end{array}$} & \multirow[t]{2}{*}{$\begin{array}{l}\text { Loseit! (SM } \\
\text { app) }\end{array}$} & $\begin{array}{l}\text { AM: } \downarrow \text { Sig. in weight, }(97.3 \pm 22.5 \mathrm{~kg} \text { vs } \\
95.0 \pm 22.1 \mathrm{~kg} \text {; small effect size, Cohen's } \\
\mathrm{d}=0.1, \mathrm{p}=0.000) \\
\downarrow \text { Sig. in BMI ( } 36.4 \pm 8.1 \mathrm{~kg} / \mathrm{m}^{2} \text { vs } 35.6 \pm \\
8.0 \mathrm{~kg} / \mathrm{m}^{2}, \text { small effect size, Cohen's } \mathrm{d}= \\
0.09, \mathrm{p}=0.0000) \\
\downarrow \text { Sig. in WC }(106.6 \pm 16.8 \mathrm{~cm} \text { vs } 103.4 \\
\pm 17.4 \mathrm{~cm}, \text { small effect size, Cohen's } \mathrm{d}= \\
0.2, p=0.0006)\end{array}$ & \multirow[t]{2}{*}{$\begin{array}{l}\text { Social } \\
\text { cognitiv } \\
\text { e theory } \\
\text { and } \\
\text { Theory } \\
\text { of } \\
\text { Planned } \\
\text { Behavio } \\
\text { r used } \\
\text { in app }\end{array}$} \\
\hline & & & & & & & App usage not reported & \\
\hline 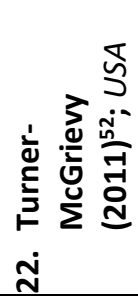 & $\begin{array}{l}\mathrm{n}=96 \\
\text { Age } 18-60 y \mathrm{rs} \\
\text { BMI } 25- \\
45 \mathrm{~kg} / \mathrm{m}^{2}\end{array}$ & $\mathrm{RCT}$ & $\begin{array}{l}2 \text { groups: } \mathbf{I} \mathbf{1}(\mathbf{n}=\mathbf{4 9}) \text { Podcast } \\
\text { only. Received } 2 \text { podcasts/week } \\
\text { (15 min) \& } 2 \text { mini } \\
\text { podcasts/week }(5 \mathrm{~min}) \& \text { a book } \\
\text { with calorie \& fat gram } \\
\text { amounts. }\end{array}$ & $\begin{array}{l}6 \\
\text { months }\end{array}$ & Obesity & $\begin{array}{l}\text { Fatsecret's } \\
\text { (SM app) }\end{array}$ & $\begin{array}{l}\text { F/N-RH: No Sig diff in energy intakes, } \\
\text { and fat intakes from baseline (small } \\
\text { effect sizes, Cohen's } d<0.1 \text { ) } \\
\text { AM: No sig diff in weight change in both } \\
\text { interventions ( } 11:-2.7 \pm 5.1 \mathrm{Kg} \mathbf{1 2}:-2.7 \pm \\
5.6 \mathrm{Kg} \text {, small effect size, Cohen's } d=0 \text {, } \\
p=0.88 \text { ) }\end{array}$ & $\begin{array}{l}\text { Social } \\
\text { Cognitiv } \\
\text { e } \\
\text { Theory } \\
\text { (podcas } \\
\text { ts only) }\end{array}$ \\
\hline
\end{tabular}




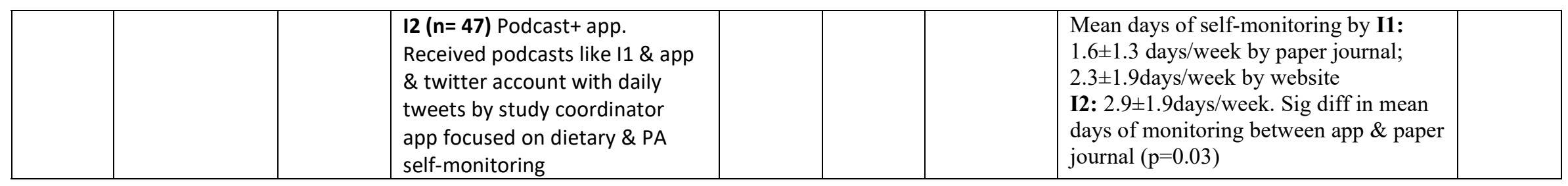

${ }^{1}$ BMI: Body Mass Index;

${ }^{2}$ I: Intervention Group (I1: Intervention group 1, I2: Intervention group 2; I3: Intervention group 3)

${ }^{3}$ SM app: Mobile app focused on self-monitoring

${ }^{4}$ AM: Anthropometric Measurements

${ }^{5} \downarrow$ Sig: Significant decrease

${ }^{6} \mathrm{C}$ : Control group

${ }^{7}$ CVD: Cardiovascular Diseases

${ }^{8} \mathrm{ED}$ app: mobile app focused on education

${ }^{9}$ F/N-RH: Food/Nutrition Related History

$10 \uparrow$ Sig: Significant increase

${ }^{11}$ No sig. diff: No significant difference

${ }^{12}$ PA: Physical Activity

${ }^{13}$ SCT: Social Cognitive Theory

${ }^{14}$ DMDMG: Diabetes Mellitus Dietary Management Guide

${ }^{15}$ PSDCS: Patient-centered smartphone-based diabetes care system

${ }^{16}$ BD/MT \&P: Biochemical Data, Medical Tests \& procedures

${ }^{17}$ WC: Waist Circumference

${ }^{18}$ SBP: Systolic Blood Pressure

${ }^{19}$ DBP: Diastolic Blood Pressure

${ }^{20}$ MI: Motivational Interviewing

${ }^{21}$ MMM: MyMealMate

${ }^{22}$ TPB: Theory of Planned Behavior 
Table 3: Quality Criteria Checklist of the articles included from a systematic review assessing the effectiveness of dietary mobile applications on nutritional outcomes in adults with chronic diseases

\begin{tabular}{|c|c|c|c|c|c|c|c|c|c|c|c|c|}
\hline \multirow[t]{2}{*}{ Author/Year } & \multirow{2}{*}{$\begin{array}{l}\text { Quality } \\
\text { Rating }\end{array}$} & \multicolumn{10}{|c|}{ Validity Questions } & \multirow[b]{2}{*}{ Comments/Study Limitations } \\
\hline & & $1^{1}$ & $2^{2}$ & $3^{3}$ & $4^{4}$ & $5^{5}$ & $6^{6}$ & $7^{7}$ & $8^{8}$ & $9^{9}$ & $\begin{array}{c}10 \\
10\end{array}$ & \\
\hline \multicolumn{13}{|c|}{ Mobile apps as the sole intervention } \\
\hline Carter $(2017)^{58}$ & Neutral & $\begin{array}{c}\mathrm{Y} \\
11\end{array}$ & $\begin{array}{c}\mathrm{n} / \mathrm{a} \\
12\end{array}$ & $\mathrm{n} / \mathrm{a}$ & $\mathrm{Y}$ & $\begin{array}{l}\mathrm{U} \\
13\end{array}$ & $\mathrm{Y}$ & $\mathrm{Y}$ & $\mathrm{Y}$ & $\mathrm{Y}$ & $\mathrm{Y}$ & $\begin{array}{l}\text { Post-hoc analysis of an original study } \\
\text { for app users (investigation of self- } \\
\text { monitoring and weight loss) }\end{array}$ \\
\hline Eyles $(2017)^{62}$ & Positive & $\mathrm{Y}$ & $\mathrm{Y}$ & Y & $\mathrm{Y}$ & Y & $\mathrm{U}$ & $\mathrm{Y}$ & $\mathrm{Y}$ & $\mathrm{Y}$ & $\mathrm{Y}$ & $\begin{array}{l}\text { Food purchases rather than food } \\
\text { intakes were considered outcomes } \\
\text { measures }\end{array}$ \\
\hline Gilson $(2017)^{50}$ & Neutral & $\mathrm{Y}$ & $\mathrm{U}$ & $\mathrm{n} / \mathrm{a}$ & $\mathrm{Y}$ & $\mathrm{n} / \mathrm{a}$ & $\mathrm{Y}$ & $\mathrm{Y}$ & $\mathrm{U}$ & $\mathrm{Y}$ & $\mathrm{Y}$ & $\begin{array}{l}\text { Bias generated due to study design } \\
\text { (lack of power analysis, feasibility } \\
\text { sampling) }\end{array}$ \\
\hline Mummah $(2017)^{51}$ & Positive & $\mathrm{Y}$ & $\mathrm{Y}$ & $\mathrm{Y}$ & $\mathrm{Y}$ & $\mathrm{Y}$ & $\mathrm{Y}$ & $\mathrm{Y}$ & $\mathrm{Y}$ & $\mathrm{Y}$ & $\mathrm{Y}$ & Study of 2 months duration \\
\hline $\begin{array}{l}\text { Turner-McGrievy, } \\
\text { G. }(2017)^{54}\end{array}$ & Positive & $\mathrm{Y}$ & $\mathrm{Y}$ & $\mathrm{Y}$ & $\mathrm{Y}$ & $\mathrm{Y}$ & $\mathrm{Y}$ & $\mathrm{Y}$ & $\mathrm{Y}$ & $\mathrm{Y}$ & $\mathrm{Y}$ & No comments to report \\
\hline Ahn $(2016)^{59}$ & Neutral & $\mathrm{Y}$ & $\mathrm{N}^{14}$ & $\bar{U}$ & $\mathrm{n} / \mathrm{a}$ & $\mathrm{N}$ & $\mathrm{U}$ & $\mathrm{Y}$ & $\mathrm{Y}$ & $\mathrm{Y}$ & $\mathrm{Y}$ & $\begin{array}{l}\text { Bias generated due to study design } \\
\text { (development study, convenience } \\
\text { sample) }\end{array}$ \\
\hline $\operatorname{Kim}(2016)^{60}$ & Neutral & $\mathrm{Y}$ & $\mathrm{U}$ & $\mathrm{n} / \mathrm{a}$ & $\mathrm{N}$ & $\mathrm{n} / \mathrm{a}$ & $\mathrm{Y}$ & $\mathrm{U}$ & $\mathrm{Y}$ & $\mathrm{Y}$ & $\mathrm{U}$ & $\begin{array}{l}\text { Bias generated due to study design } \\
\text { (recruitment, duration, withdrawal } \\
\text { descriptions, selected nutrition } \\
\text { measures were not specific to } \\
\text { outcomes of concern, conflict of } \\
\text { interest) }\end{array}$ \\
\hline Mummah (2016) & Neutral & $\mathrm{Y}$ & $\mathrm{Y}$ & $\mathrm{Y}$ & $Y$ & $Y$ & $\mathrm{Y}$ & $\mathrm{Y}$ & $Y$ & $\mathrm{Y}$ & $Y$ & $\begin{array}{l}\text { Bias was generated due to study } \\
\text { design (participants were recruited } \\
\text { from an ongoing weight loss parent } \\
\text { trial) }\end{array}$ \\
\hline
\end{tabular}




\begin{tabular}{|c|c|c|c|c|c|c|c|c|c|c|c|c|}
\hline Willey $(2016)^{55}$ & Neutral & $\mathrm{Y}$ & $\mathrm{Y}$ & $\mathrm{n} / \mathrm{a}$ & $\mathrm{n} / \mathrm{a}$ & $\mathrm{n} / \mathrm{a}$ & $\mathrm{Y}$ & $\mathrm{Y}$ & $\mathrm{Y}$ & $\mathrm{Y}$ & $\mathrm{N}$ & $\begin{array}{l}\text { Bias generated due to study design } \\
\text { (lack of power analysis, conflict of } \\
\text { interest) }\end{array}$ \\
\hline Laing $(2014)^{56}$ & Positive & $\mathrm{Y}$ & $\mathrm{Y}$ & $\mathrm{Y}$ & $\mathrm{Y}$ & $\mathrm{N}$ & $\mathrm{Y}$ & $\mathrm{Y}$ & $\mathrm{U}$ & $\mathrm{Y}$ & $\mathrm{Y}$ & $\begin{array}{l}\text { Some control subject used the app due } \\
\text { to its public availability }\end{array}$ \\
\hline Kirwan $(2013)^{61}$ & Neutral & $\mathrm{Y}$ & $\mathrm{Y}$ & $\mathrm{Y}$ & $\mathrm{Y}$ & $\mathrm{N}$ & $\mathrm{Y}$ & $\mathrm{U}$ & $\mathrm{Y}$ & $\mathrm{Y}$ & $\mathrm{Y}$ & $\begin{array}{l}\text { Selected nutrition measures were not } \\
\text { specific to outcomes of concern }\end{array}$ \\
\hline $\begin{array}{l}\text { Turner-McGrievy, } \\
\text { G. }(2013)^{53}\end{array}$ & Neutral & $\mathrm{Y}$ & $\mathrm{n} / \mathrm{a}$ & $\mathrm{n} / \mathrm{a}$ & $\mathrm{N}$ & $\mathrm{N}$ & $\mathrm{Y}$ & $\bar{Y}$ & $\mathrm{Y}$ & $\mathrm{Y}$ & $\mathrm{Y}$ & $\begin{array}{l}\text { Secondary analysis of Turner- } \\
\text { McGrievy, G. (2011) }\end{array}$ \\
\hline \multicolumn{13}{|c|}{ Comprehensive Interventions (multiple arms) with one arm mobile app as the sole intervention } \\
\hline Burke $(2017)^{46}$ & Neutral & $\mathrm{Y}$ & $\mathrm{Y}$ & $\mathrm{Y}$ & $\mathrm{Y}$ & $\mathrm{N}$ & $\mathrm{Y}$ & $\mathrm{Y}$ & $\mathrm{U}$ & $\mathrm{Y}$ & $\mathrm{Y}$ & $\begin{array}{l}\text { Bias generated due to study design } \\
\text { (pilot trial, lack of power analysis) }\end{array}$ \\
\hline $\operatorname{Ross}(2016)^{57}$ & Neutral & $\mathrm{Y}$ & $\mathrm{Y}$ & $\mathrm{Y}$ & $\mathrm{U}$ & $\mathrm{U}$ & $\mathrm{Y}$ & $\mathrm{Y}$ & $\mathrm{Y}$ & $\mathrm{Y}$ & $\mathrm{Y}$ & $\begin{array}{l}\text { Bias generated due to study design } \\
\text { (pilot trial) }\end{array}$ \\
\hline Holmen $(2014)^{45}$ & Neutral & $\mathrm{Y}$ & $\mathrm{U}$ & $\mathrm{Y}$ & $\mathrm{Y}$ & $\mathrm{N}$ & $\mathrm{Y}$ & $\mathrm{Y}$ & $\mathrm{Y}$ & $\mathrm{Y}$ & $\mathrm{Y}$ & No intention to treat analysis \\
\hline Allen $(2013)^{43}$ & Neutral & Y & $\mathrm{Y}$ & $\mathrm{Y}$ & $\mathrm{Y}$ & $\mathrm{N}$ & $\mathrm{Y}$ & $\mathrm{Y}$ & $\mathrm{Y}$ & $\mathrm{Y}$ & $\mathrm{Y}$ & $\begin{array}{l}\text { Bias generated due to study design } \\
\text { (pilot study, lack of power analysis, no } \\
\text { intention to treat analysis) }\end{array}$ \\
\hline Carter $(2013)^{49}$ & Positive & Y & Y & Y & Y & $\mathrm{N}$ & Y & $\mathrm{Y}$ & $\mathrm{Y}$ & $\mathrm{Y}$ & $\mathrm{Y}$ & Study lacked a power analysis \\
\hline \multicolumn{13}{|c|}{ Counseling Interventions supported by app } \\
\hline Stephens $(2017)^{42}$ & Positive & $\mathrm{Y}$ & $\mathrm{Y}$ & Y & $\mathrm{Y}$ & $\mathrm{N}$ & $\mathrm{Y}$ & $\mathrm{Y}$ & $\mathrm{Y}$ & $\mathrm{Y}$ & $\mathrm{Y}$ & $\begin{array}{l}\text { Nutrition measures used were valid } \\
\text { and reliable }\end{array}$ \\
\hline Hartman $(2016)^{63}$ & Neutral & $\mathrm{Y}$ & $\mathrm{U}$ & Y & $\mathrm{Y}$ & $\mathrm{N}$ & $\mathrm{Y}$ & $\mathrm{Y}$ & $\mathrm{Y}$ & $\mathrm{Y}$ & $\mathrm{Y}$ & $\begin{array}{l}\text { Bias generated due to study design } \\
\text { (pilot study, sample size selection } \\
\text { unclear) }\end{array}$ \\
\hline Block $(2015)^{44}$ & Positive & $\mathrm{Y}$ & $\mathrm{Y}$ & $\mathrm{Y}$ & $\mathrm{Y}$ & $\mathrm{N}$ & $\mathrm{Y}$ & $\mathrm{Y}$ & $\mathrm{Y}$ & $\mathrm{Y}$ & $\mathrm{Y}$ & $\begin{array}{l}\text { Mobile app was one among other } \\
\text { mhealth interventions }\end{array}$ \\
\hline McCaroll $(2015)^{64}$ & Neutral & $\mathrm{Y}$ & $\mathrm{Y}$ & $\mathrm{n} / \mathrm{a}$ & $\mathrm{U}$ & $\mathrm{N}$ & $\mathrm{Y}$ & $\mathrm{Y}$ & $\mathrm{Y}$ & $\mathrm{Y}$ & $\mathrm{Y}$ & $\begin{array}{l}\text { Bias generated due to study design } \\
\text { (feasibility study, short duration, small } \\
\text { sample) }\end{array}$ \\
\hline
\end{tabular}




\begin{tabular}{|l|l|c|c|c|c|c|c|c|c|c|c|c|}
\hline $\begin{array}{l}\text { Turner-McGrievy, } \\
\text { G. (2011) })^{52}\end{array}$ & Neutral & Y & Y & Y & N & N & Y & Y & Y & Y & Y & $\begin{array}{l}\text { Bias generated due to lack of blinding, } \\
\text { reporting of withdrawals and reasons } \\
\text { for withdrawals }\end{array}$ \\
\hline
\end{tabular}

${ }^{1} 1-$ Was the research question clearly stated?

2 -Was the selection of study subjects/patients free from bias?

${ }^{3} 3$ - Were study groups comparable?

${ }^{4} 4$-Was method of handling withdrawals described?

${ }_{5}^{5} 5$-Was blinding used to prevent introduction of bias?

${ }^{6} 6$-Were intervention/therapeutic regimens/exposure factor or procedure and any comparison(s) described in detail? Were intervening factors described?

77-Were outcomes clearly defined and the measurements valid and reliable?

8 -Was the statistical analysis appropriate for the study design and type of outcome indicators?

9 9-Are conclusions supported by results with biases and limitations taken into consideration?

${ }^{10} 10$-Is bias due to study's funding or sponsorship unlikely?

${ }^{11} \mathrm{Y}$ : Yes

${ }^{12} \mathrm{n} / \mathrm{a}$ : Not applicable

${ }^{13} \mathrm{U}$ : Unclear

${ }^{14} \mathrm{~N}: \mathrm{No}$ 


\section{COUNSELING INTERVENTIONS SUPPORTED BY APPS}

Five studies incorporated mobile apps as part of an intervention mainly consisting of in-person or phone counseling. Four of these found significant changes in all measured outcomes, with one exception [52], which found no significant differences in anthropometrics and energy and nutrient intake (energy intake, fat intake, and weight loss from baseline for both the intervention and control groups did not change). However, this study reported increased selfmonitoring among app users as compared to users of a paper journal (2.9 \pm 1 .9days/week vs $1.6 \pm 1.3$ days/week by paper journal, $\mathrm{p}=0.03$ ). All other studies in this category $[42,44,63,64]$ could detect significant improvements in anthropometric measurements as compared to control groups. In one study [63], a large effect size was identified on weight loss. This intervention also reported small but significant changes in physical activity $(+15.01 \mathrm{~min} / \mathrm{d}$ by intervention compared to $+10.9 \mathrm{~min} / \mathrm{d}$ by control group, small effect size, Cohen's $\mathrm{d}=0.33, \mathrm{p}=0.01$ ). A study [42] also reported increased weight loss with increased app engagement particularly with selfmonitoring of physical activity $(0.03 \mathrm{~kg}$ weight loss per additional day of physical activity logged, $\mathrm{p}=0.026$ ).

Only one study [44] in this category reported biochemical outcomes: significant improvements in HbA1c $(-0.26$ vs $-0.18 \%, \mathrm{p}<0.001)$ and fasting glucose $(-7.36 \mathrm{vs}-2.19 \mathrm{mg} / \mathrm{dl}, \mathrm{p}<0.001)$.

\section{RESULTS OF META-ANALYSIS}

A random-effects meta-analysis was performed for any outcome that was measured by at least two RCTs. Eleven RCTs were incorporated into the meta-analysis comparing the effects of app usage to control (no app) on weight loss [42-45, 49, 52-54, 57, 63]. Five RCTs were included for change in BMI [42-44, 49, 59], and three each for change in waist circumference [42-44], energy intake [43, 52, 54], fruit and/or vegetable intake [43, 51, 65], and Hba1c [44, 45, 61]. Table 4 shows the results of the meta-analysis of the multiple outcomes.

Pooled estimates showed a significantly greater decrease in weight, waist circumference, and energy intake when an app was used as compared to control. A net decrease of about $-2.45 \mathrm{~kg}$ ( $95 \%$ CI -3.33 to -1.58 ; $<<0.001$; I2=96.2\%, 95\% CI 95\% to 97\%) in body weight was estimated. All studies included in the analysis used a self-monitoring app and 10 studies incorporated a behavioral theory [42-45, 49, 52-54, 57, 63]. RCTs varied in duration between one month [59] and one year [45], with nine studies assessing weight loss after at least six months [42-44, 49, 52-54, 57, 63].

A net change in waist circumference of $-2.54 \mathrm{~cm}(95 \% \mathrm{CI}-3.34$ to $-1.73 ; \mathrm{p}<0.001 ; \mathrm{I} 2=88.3 \%$, $95 \%$ CI $67 \%$ to $96 \%$ ) in waist circumference and of $-149.52 \mathrm{Kcal}$ (95\% CI -215.78 to -83.27 ; $\mathrm{p}<0.001 ; \mathrm{I} 2=0 \%, 95 \%$ CI $0 \%$ to $90 \%$ ) in energy intake were estimated. Forest plots are 
presented in Figures 2 and 3 for the effects of app usage on weight loss (11 studies) and BMI (five studies), respectively.

The pooled estimates were insignificant for change in BMI, Hba1c, and fruit and/or vegetable intake. However, with app usage, the mean difference of pooled results decreased for BMI and Hbalc and increased for fruit and/or vegetable intake. All the pooled estimates show considerable heterogeneity with an I2 above $75 \%$. Change in energy intake showed an I 2 of $0 \%$; however, the $95 \%$ CI ranged from $0 \%$ to $90 \%$, thus, heterogeneity could not be excluded [41].

Table 4: Results of a random effects meta-analysis assessing the effectiveness of dietary app usage as compared to no app usage on multiple nutritional outcomes of adults with chronic diseases

\begin{tabular}{|c|c|c|c|c|c|c|}
\hline Nutritional Outcome & $\begin{array}{l}\text { RCT } \\
\text { included }\end{array}$ & $\begin{array}{l}\text { Sample } \\
\text { size } \\
\text { (APP) }\end{array}$ & $\begin{array}{l}\text { Sample } \\
\text { size (NO } \\
\text { APP) }\end{array}$ & $\begin{array}{l}\text { Total Mean } \\
\left.\text { Difference (CI }{ }^{1}\right) \\
\text { Random Effect } \\
\end{array}$ & $P$ value & $I^{2}(95 \% \mathrm{CI})$ \\
\hline \multicolumn{7}{|c|}{ Anthropometric measurement outcomes } \\
\hline Weight Loss (Kg) & 11 & 498 & 484 & $-2.45(-3.33,-1.57)$ & $\mathrm{P}<0.001 * * *$ & $\begin{array}{l}96.2 \%(95 \%- \\
97 \%)\end{array}$ \\
\hline Change in $\mathrm{BMI}^{2}\left(\mathrm{~kg} / \mathrm{m}^{2}\right)$ & 5 & 265 & 279 & $-0.32(-0.78,0.13)$ & 0.165 & $\begin{array}{l}89.6 \%(79 \%- \\
95 \%)\end{array}$ \\
\hline $\begin{array}{l}\text { Change in Waist } \\
\text { Circumference }(\mathrm{cm})\end{array}$ & 3 & 208 & 224 & $-2.54(-3.34,-1.73)$ & $\mathrm{P}<0.001 * * *$ & $\begin{array}{l}88.3 \%(67 \%- \\
96 \%)\end{array}$ \\
\hline \multicolumn{7}{|c|}{ Food-/nutrition-related outcomes } \\
\hline $\begin{array}{l}\text { Change in Energy Intakes } \\
\text { (Kcal/d) }\end{array}$ & 3 & 105 & 106 & $\begin{array}{l}-149.52(-215.78, \\
-83.27)\end{array}$ & $\mathrm{P}<0.001 * * *$ & $\begin{array}{l}0 \%(0 \%- \\
90 \%)\end{array}$ \\
\hline $\begin{array}{l}\text { Change in } \\
\text { Fruits/Vegetables intakes } \\
\text { (servings per day) }\end{array}$ & 3 & 92 & 94 & $4.13(-0.45,8.71)$ & 0.77 & $\begin{array}{l}95.9 \%(91 \%- \\
98 \%)\end{array}$ \\
\hline \multicolumn{7}{|c|}{ Clinical/biochemical data outcomes } \\
\hline Change in Hbalc $(\%)$ & 3 & 221 & 240 & $-0.54(-1.38,0.3)$ & 0.207 & $\begin{array}{l}99.3 \%(99 \%- \\
100 \%)\end{array}$ \\
\hline
\end{tabular}

${ }^{1} \mathrm{CI}$ : Confidence Interval

${ }^{2}$ BMI: Body Mass Index 


\section{Forest Plot (Weight Loss in Kg)}

Study

ID

$\%$
$\operatorname{WMD}(95 \% \mathrm{Cl}) \quad$ Weight

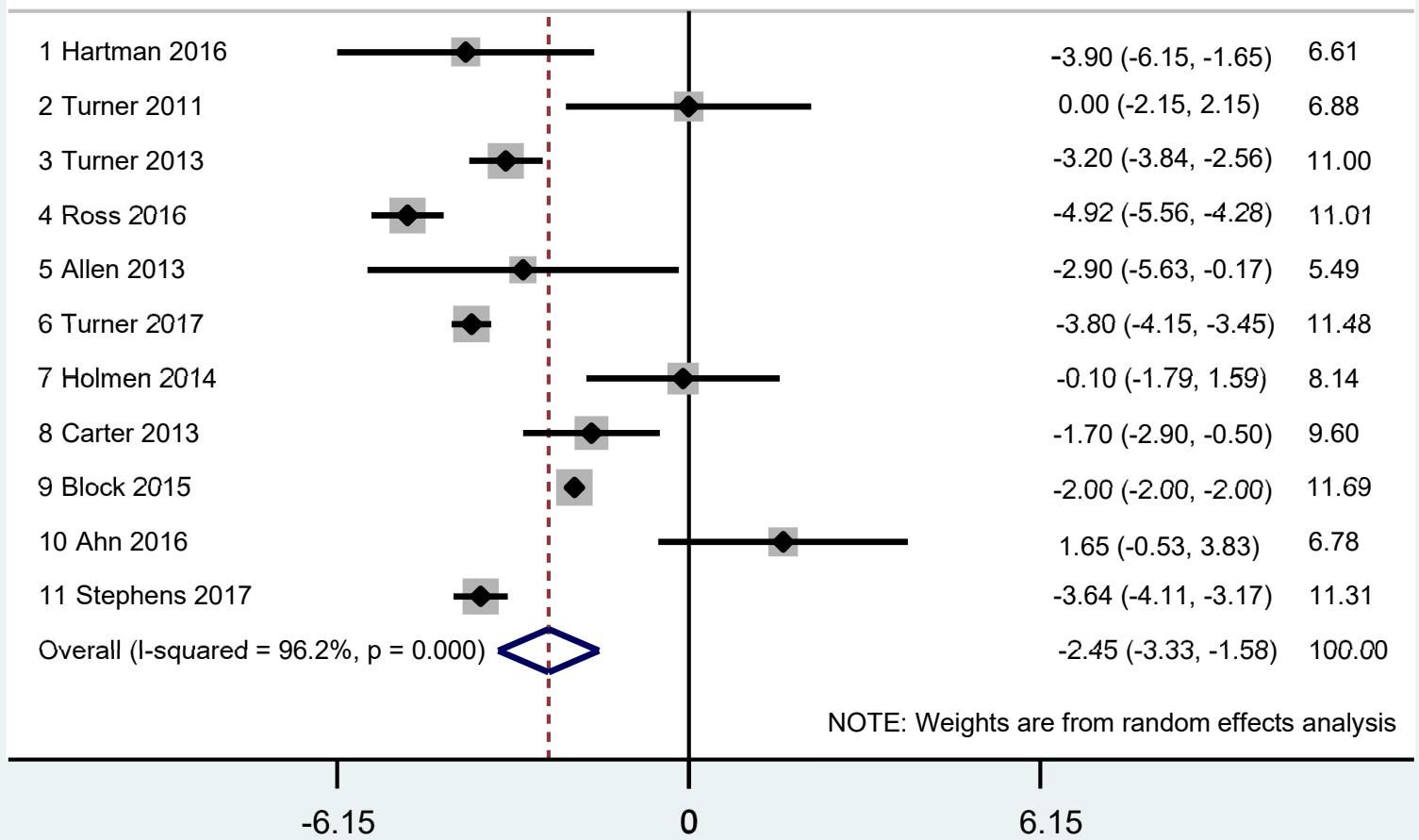

Figure 2: Forest plot of a random effects meta-analysis assessing the effectiveness of dietary application (app) usage compared to no app usage on weight loss among adults with chronic diseases. WMD1/4weighted mean difference. 
Study

$\%$

ID

WMD $(95 \% \mathrm{Cl}) \quad$ W'eight

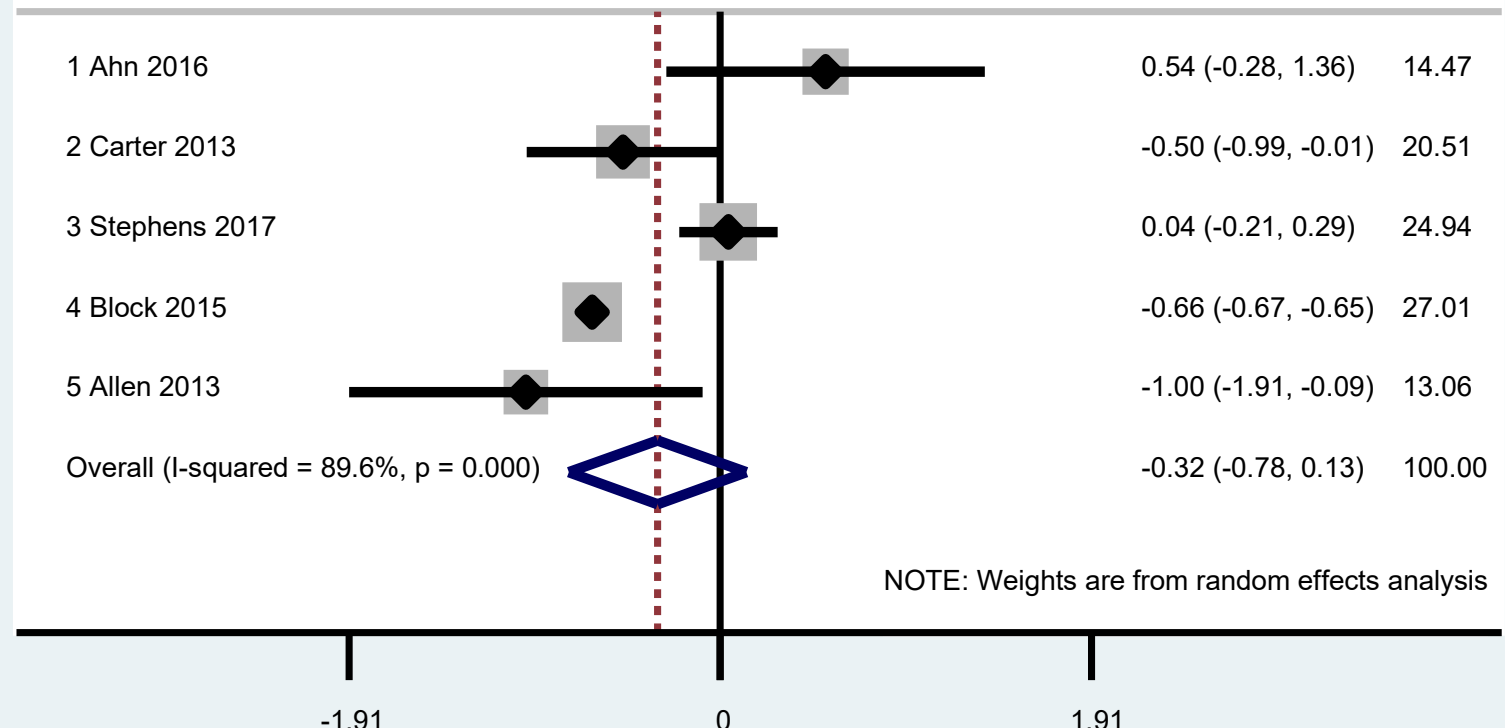

Figure 3: Forest plot of a random effects meta-analysis assessing the effectiveness of dietary application (app) usage compared to no app usage on change in body mass index (BMI) among adults with chronic diseases. WMD1/4weighted mean difference

\section{DISCUSSION}

This review included interventional studies assessing the impact of the use of dietary mobile apps on nutritional outcomes in adults with chronic diseases. More than $75 \%$ of the included studies found a positive change among app users in at least one of the nutritional outcomes measured while more than $50 \%$ detected a medium or large effect size in the change of the measured outcomes.

Pooled estimates derived from the random-effects meta-analysis show a clear positive effect of self-monitoring dietary apps on weight loss as compared to other self-monitoring tools (such as paper-based diaries [49, 52, 57]), other technologies (bite counter [54]), or no selfmonitoring tools $[43,44,59]$. The heterogeneity of data does not allow strong conclusions to be drawn [66]. The results still need to be interpreted with caution owing to the differences in comparators, duration of the interventions, and inclusion or absence of counseling. 
The majority of RCTs (10 studies [42-45, 49, 52-54, 57, 63]) included in the pooled estimate analysis for weight loss incorporated behavioral theories or their constructs, and more than half (seven studies [42-44, 52-54,63]) consisted of a counseling intervention using an app. Additionally, all three RCTs used in the pooled analysis for Hba1c consisted of a counseling intervention $[44,45,61]$. Given that the results of the pooled estimates all favored app usage, it may be suggested that incorporating a behavioral theory and/or using apps in counseling interventions is an effective strategy.

Nine studies evaluated commercial self-monitoring apps [42, 43, 46, 49, 50, 52-54, 64] available to the public on app stores, rather than apps developed for research purposes. Despite the positive findings of this review and meta-analysis, the quality and effectiveness of commercial apps is questionable. The potential benefits and safety concerns of commercial dietary mobile apps have not been investigated thoroughly. While legislators across the world have started to consider app regulations in the healthcare setting [67], in the meantime, many apps are already in use without proof of their quality. A content analysis of popular weight management apps available in app stores raises concerns about the quality of commercially available apps. The main concern was that apps do not include enough behavioral theory-based strategies [68].

Unlike a study that identified five key components that render technology-based interventions efficacious [69], this systematic review was not able to provide evidence to support characteristics that render dietary mobile apps efficacious. However, it identified that greater app usage may lead to better outcomes. For instance, in an RCT, [52] no significant changes in outcomes were reported. When participants were re-clustered by the self-monitoring method [53], the authors detected significant changes, with medium effect sizes, on BMI and weight among app users using the physical activity tracking feature $(-3.7 \pm 1.5 \%$ vs non-physical activity app users $-0.5 \pm 1.5 \% ; \mathrm{p}=0.01)$. A study [58] also performed a post-hoc analysis of app users and found that HFUs lost significantly more weight as compared to moderate-frequency users (MFUs) and LFUs (HFUs were $-4.7 \mathrm{~kg}$ lower than MFUs and $-6.4 \mathrm{~kg}$ lower than LFUs, $\mathrm{p}<0.001)$.

Interventions with dietary apps targeting diabetes or pre-diabetes, irrespective of the study design, found significant improvements in both food/nutrition-related history [59-61] and HbA1c $[44,60]$. Pooled estimates were calculated from three RCTs $[44,45,61]$ and the results, despite being statistically insignificant, also favor app usage. One of the three studies [45] did not find favorable outcomes of dietary apps in the management of diabetes as compared to usual care. This was a year-long intervention that took place in Norway. While the authors did not describe usual care, according to Norway's directorate of health [70], the country counts it 
among its national goals to be a pioneer in the prevention and management of diabetes. Therefore, it is possible that usual care, per se, was very effective, which makes it necessary to view this intervention [45] within its national context. The aforementioned study is the only one involving a year-long intervention, which makes it necessary for future research to investigate the effectiveness of dietary mobile apps in the longer term. Thus, while the effect of dietary apps on Hbalc also seems promising, more data are needed to support such conclusions.

Dietary mobile apps are effective self-monitoring tools that support weight loss in the short term, but more research is needed to draw conclusions regarding other nutritional outcomes. Further research should evaluate the long-term effects of dietary mobile apps, as well as assessing their content and dependability, particularly in the case of commercially available apps. While educational dietary apps seem promising, not enough research is available in this field. More research on the role of dietary mobile apps in chronic diseases other than obesity and diabetes would significantly contribute to clinical practice.

\section{LIMITATIONS}

This systematic review is subject to large publication bias, as unpublished interventions were not included. Publication bias tests have not performed due to the small number of studies included in the meta-analysis. However, most included articles have been published recently, thus we predict publication bias to be small [71]. The main limitation of this review is the lack of available studies exploring the use of mobile apps in chronic conditions other than obesity and diabetes. While some of the identified apps target cancer, they mainly aim at weight loss as part of cancer prevention and treatment. Accordingly, the results of this review are also subject to limited generalizability across chronic diseases. A gap in the knowledge about the effectiveness of dietary mobile apps in conditions that require extensive dietary management and behavioral change, such as chronic kidney disease and cardiovascular diseases, still exists. Another limitation is that most apps consisted of self-monitoring tools; only two studies included educational apps $[55,60]$, and they both found positive outcomes regarding food/nutrition-related history, anthropometry, and/or biochemical/clinical parameters. Therefore, most conclusions pertain to self-monitoring by means of an app.

Additionally, this review did not intend to evaluate the content of the apps and benchmark their compliance with available dietary evidence-based practice. Available content analysis investigating the incorporation of behavioral theories into apps also indicates a lack of compliance [68]. Accordingly, it is advisable that further research explore the quality of commercial apps. 
It is essential to note that all studies took place in developed countries, resulting in conclusions not generalizable at the global level, and especially to developing countries. Mobile apps may provide effective outcomes in areas with less access to in-person healthcare, and thus further research in developing countries and rural areas would be informative. Dietary mobile apps are incorporated into smartphones and tablets, generating a bias related to their affordability and making this technology accessible only to those who can afford it. The heterogeneity of the data used in the meta-analysis, making it harder to draw strong conclusions based on the results [66], adds another limitation.

The results of this review and meta-analysis are only applicable in the short term as most interventions lasted between three and six months, with only one year-long study [45]. Therefore, more studies should address the long-term effects of dietary apps with a follow-up longer than six months.

\section{CONCLUSION}

This systematic review and meta-analysis demonstrated that dietary mobile apps used for selfmonitoring have positive effects on nutritional outcomes in the short-term, particularly weight loss, waist circumference, and energy intake. Dietary apps that include a behavioral theory incorporated into a counseling intervention may provide additional benefits, especially in the case of weight management.

\section{ACKNOWLEDGEMENTS}

The authors acknowledge the work of Aida Farha and her input and revision of the search strategy used in this systematic review. 


\section{REFERENCES}

1. World Health Organization. Health in 2015: From MDGs, millennium development goals to SDGs, sustainable development goals: World Health Organization; 2015.

2. Slawson DL, Fitzgerald N, Morgan KT. Position of the Academy of Nutrition and Dietetics: the role of nutrition in health promotion and chronic disease prevention. J Acad Nutr Diet. 2013;113:972-979.

3. Warkentin LM, Das D, Majumdar SR, Johnson JA, Padwal RS. The effect of weight loss on health-related quality of life: systematic review and meta-analysis of randomized trials. Obes Rev. 2014;15:169-182.

4. Nowson CA, Service C, Appleton J, Grieger JA. The impact of dietary factors on indices of chronic disease in older people: A systematic review. J Nutr Health Aging. 2017.

5. Karavetian M, de Vries N, Rizk R, Elzein H. Dietary educational interventions for management of hyperphosphatemia in hemodialysis patients: a systematic review and meta-analysis. Nutr Rev. 2014;72:471-482.

6. Sun Y, You W, Almeida F, Estabrooks P, Davy B. The Effectiveness and Cost of Lifestyle Interventions Including Nutrition Education for Diabetes Prevention: A Systematic Review and Meta-Analysis. J Acad Nutr Diet. 2017;117:404-421.e436.

7. Rizk R, Karavetian M, Hiligsmann M, Evers S. Effect of stage-based education provided by dedicated dietitians on hyperphosphataemic haemodialysis patients: results from the Nutrition Education for Management of Osteodystrophy randomised controlled trial. J Hum Nutr Diet. 2017;30:554-562.

8. Kirac M, Kupeli B, Irkilata L, et al. Effects of dietary interventions on 24-hour urine parameters in patients with idiopathic recurrent calcium oxalate stones. Kaohsiung J Med Sci. 2013;29:88-92.

9. Mitchell LJ, Ball LE, Ross LJ, Barnes KA, Williams LT. Effectiveness of Dietetic Consultations in Primary Health Care: A Systematic Review of Randomized Controlled Trials. J Acad Nutr Diet. 2017.

10. Raynor HA, Davidson PG, Burns H, et al. Medical Nutrition Therapy and Weight Loss Questions for the Evidence Analysis Library Prevention of Type 2 Diabetes Project: Systematic Reviews. J Acad Nutr Diet. 2017;117:1578-1611.

11. Wolf AM, Siadaty M, Yaeger B, et al. Effects of lifestyle intervention on health care costs: Improving Control with Activity and Nutrition (ICAN). J Am Diet Assoc. 2007; 107:1365-1373.

12. Rizk R, Hiligsmann M, Karavetian M, Evers S. Cost-effectiveness of dedicated dietitians for hyperphosphatemia management among hemodialysis patients in Lebanon: results 
from the Nutrition Education for Management of Osteodystrophy trial. J Med Econ. 2017;20:1024-1038.

13. Greaves CJ, Sheppard KE, Abraham C, et al. Systematic review of reviews of intervention components associated with increased effectiveness in dietary and physical activity interventions. BMC Public Health. 2011;11:119.

14. Gresham E, Bisquera A, Byles JE, Hure AJ. Effects of dietary interventions on pregnancy outcomes: a systematic review and meta-analysis. Matern Child Nutr. 2016;12:5-23

15. Gresham E, Byles JE, Bisquera A, Hure AJ. Effects of dietary interventions on neonatal and infant outcomes: a systematic review and meta-analysis. Am J Clin Nutr. 2014;100:1298-1321.

16. Spahn JM, Reeves RS, Keim KS, et al. State of the evidence regarding behavior change theories and strategies in nutrition counseling to facilitate health and food behavior change. J Am Diet Assoc. 2010;110:879-891.

17. Brautigam-Ewe M, Lydell M, Mansson J, Johansson G, Hildingh C. Dietary advice on prescription: experiences with a weight reduction programme. J Clin Nurs. 2017;26:795804.

18. Di Noia J, Furst G, Park K, Byrd-Bredbenner C. Designing culturally sensitive dietary interventions for African Americans: review and recommendations. Nutr Rev. 2013;71:224-238.

19. Pignone MP, Ammerman A, Fernandez L, et al. Counseling to promote a healthy diet in adults: a summary of the evidence for the US Preventive Services Task Force. Am J Prev Med. 2003;24:75-92.

20. Jakicic JM, King WC, Marcus MD, et al. Short-term weight loss with diet and physical activity in young adults: The IDEA study. Obesity (Silver Spring). 2015;23:2385-2397.

21. Almunawar MN, Anshari M, Younis MZ. Incorporating customer empowerment in mobile health. Health Policy Technol. 2015;4:312-319.

22. Bradway M, Arsand E, Grottland A. Mobile Health: Empowering patients and driving change. Trends Endocrinol Metab. 2015;26:114-117.

23. Park LG, Beatty A, Stafford Z, Whooley MA. Mobile Phone Interventions for the Secondary Prevention of Cardiovascular Disease. Prog Cardiovasc Dis. 2016;58:639650 .

24. DiFilippo KN, Huang WH, Andrade JE, Chapman-Novakofski KM. The use of mobile apps to improve nutrition outcomes: A systematic literature review. J Telemed Telecare. 2015;21:243-253. 
25. Sherwood NE, Morton N, Jeffery RW, French SA, Neumark-Sztainer D, Falkner NH. Consumer Preferences in Format and Type of Community-Based Weight Control Programs. Am J Health Promot. 2016;13:12-18.

26. Li X, Xu ZR, Tang N, et al. Effect of intervention using a messaging app on compliance and duration of treatment in orthodontic patients. Clin Oral Investig. 2016;20:1849-1859.

27. Boyce B. Nutrition apps: opportunities to guide patients and grow your career. J Acad Nutr Diet. 2014;114:13-15.

28. Stein K. Remote Nutrition Counseling: Considerations in a New Channel for Client Communication. J Acad Nutr Diet. 2015;115:1561-1576.

29. Poushter J. Smartphone Ownership and Internet Usage Continues to Climb in Emerging Economies But advanced economies still have higher rates of technology use: Pew Research Center; 2016.

30. Bhuyan SS, Lu N, Chandak A, et al. Use of Mobile Health Applications for HealthSeeking Behavior Among US Adults. J Med Syst. 2016;40:153.

31. Schoeppe S, Alley S, Van Lippevelde W, et al. Efficacy of interventions that use apps to improve diet, physical activity and sedentary behaviour: a systematic review. Int J Behav Nutr Phys Act. 2016;13:127.

32. Porter J, Huggins CE, Truby H, Collins J. The Effect of Using Mobile TechnologyBased Methods That Record Food or Nutrient Intake on Diabetes Control and Nutrition Outcomes: A Systematic Review. Nutrients. 2016;8.

33. Lai J, Porter J. Dietary mobile apps and their effect on nutritional indicators in chronic renal disease: a systematic review. Nephrology (Carlton). 2015.

34. Moher D, Shamseer L, Clarke M, et al. Preferred reporting items for systematic review and meta-analysis protocols (PRISMA-P) 2015 statement. Syst Rev. 2015;4:1.

35. Adolph M. ITU-T TechWatch Alert (July 2009) Mobile Applications. Vol 2017: International Telecommunications Union; 2009.

36. Lacey K, Pritchett E. Nutrition Care Process and Model: ADA adopts road map to quality care and outcomes management. J Am Diet Assoc. 2003;103:1061-1072.

37. Academy of Nutrition and Dietetics EAL. Evidence Analysis Manual: Steps in the Academy Evidence Analysis Process. In: Research IaSBDT, ed. Chicago, IL 606066995: Academy of Nutrition and Dietetics; 2016.

38. Thompson B. What future quantitative social science research could look like: Confidence intervals for effect sizes. Educ Res. 2002;31:25-32.

39. Lee DK. Alternatives to P value: confidence interval and effect size. Korean J Anesthesiol. 2016;69:555-562. 
40. DerSimonian R, Laird N. Meta-analysis in clinical trials revisited. Contemp Clin Trials. 2015;45:139-145.

41. Ioannidis JP, Patsopoulos NA, Evangelou E. Uncertainty in heterogeneity estimates in meta-analyses. BMJ. 2007;335:914.

42. Stephens JD, Yager AM, Allen J. Smartphone Technology and Text Messaging for Weight Loss in Young Adults: A Randomized Controlled Trial. J Cardiovasc Nurs. 2017;32:39-46.

43. Allen JK, Stephens J, Dennison Himmelfarb CR, Stewart KJ, Hauck S. Randomized controlled pilot study testing use of smartphone technology for obesity treatment. J Obes. 2013;2013 (no pagination).

44. Block G, Azar KM, Romanelli RJ, et al. Diabetes Prevention and Weight Loss with a Fully Automated Behavioral Intervention by Email, Web, and Mobile Phone: A Randomized Controlled Trial Among Persons with Prediabetes. J Med Internet Res. 2015; $17: \mathrm{e} 240$.

45. Holmen H, Torbjornsen A, Wahl AK, et al. A mobile health intervention for selfmanagement and lifestyle change for persons with type 2 diabetes, part 2: One-year results from the Norwegian randomized controlled trial RENEWING HEALTH. Diabetes Technol Ther. 2016;18:S58-S59.

46. Burke LE, Zheng Y, Ma Q, et al. The SMARTER pilot study: Testing feasibility of realtime feedback for dietary self-monitoring. Prev Med Rep. 2017;6:278-285.

47. StataCorp S. Release 11. Statistical Software College Station, TX: StataCorp LP. 2009.

48. Sterne JAC, Gavaghan D, Egger M. Publication and related bias in meta-analysis: Power of statistical tests and prevalence in the literature. J Clin Epidemiol. 2000;53:1119-1129.

49. Carter MC, Burley VJ, Nykjaer C, Cade JE. Adherence to a smartphone application for weight loss compared to website and paper diary: pilot randomized controlled trial. J Med Internet Res. 2013;15:e32.

50. Gilson ND, Pavey TG, Wright OR, et al. The impact of an $\mathrm{m}-\mathrm{Health}$ financial incentives program on the physical activity and diet of Australian truck drivers. BMC Public Health. 2017;17:467.

51. Mummah S, Robinson TN, Mathur M, Farzinkhou S, Sutton S, Gardner CD. Effect of a mobile app intervention on vegetable consumption in overweight adults: a randomized controlled trial. Int J Behav Nutr Phys Act. 2017;14:125.

52. Turner-McGrievy G, Tate D. Tweets, Apps, and Pods: Results of the 6-month Mobile Pounds Off Digitally (Mobile POD) randomized weight-loss intervention among adults. J Med Internet Res. 2011;13:e120. 
53. Turner-McGrievy GM, Beets MW, Moore JB, Kaczynski AT, Barr-Anderson DJ, Tate DF. Comparison of traditional versus mobile app self-monitoring of physical activity and dietary intake among overweight adults participating in an mHealth weight loss program. J Am Med Inform Assoc. 2013;20:513-518.

54. Turner-McGrievy GM, Wilcox S, Boutte A, et al. The Dietary Intervention to Enhance Tracking with Mobile Devices (DIET Mobile) Study: A 6-Month Randomized Weight Loss Trial. Obesity (Silver Spring). 2017;25:1336-1342.

55. Willey S, Walsh JK. Outcomes of a Mobile Health Coaching Platform: 12-Week Results of a Single-Arm Longitudinal Study. JMIR MHealth and UHealth. 2016;4:e3.

56. Laing BY, Mangione CM, Tseng CH, et al. Effectiveness of a smartphone application for weight loss compared with usual care in overweight primary care patients. Ann Intern Med. 2014;161:S5-S12.

57. Ross KM, Wing RR. Impact of newer self-monitoring technology and brief phone-based intervention on weight loss: A randomized pilot study. Obesity (Silver Spring). 2016;24:1653-1659.

58. Carter MC, Burley VJ, Cade JE. Weight Loss Associated With Different Patterns of SelfMonitoring Using the Mobile Phone App My Meal Mate. JMIR MHealth and UHealth. 2017;5:e8.

59. Ahn Y, Bae J, Kim HS. The development of a mobile u-Health program and evaluation for self-diet management for diabetic patients. Nutrition Research \& Practice. 2016;10:342-351.

60. Kim EK, Kwak SH, Baek S, et al. Feasibility of a Patient-Centered, Smartphone-Based, Diabetes Care System: A Pilot Study. Diabetes Metab J. 2016;40:192-201.

61. Kirwan M, Vandelanotte C, Fenning A, Duncan MJ. Diabetes self-management smartphone application for adults with type 1 diabetes: randomized controlled trial. J Med Internet Res. 2013;15:e235.

62. Eyles H, Neal B, Jiang Y, Doughty RN, McLean R, Ni Mhurchu C. A salt-reduction smartphone app supports lower-salt food purchases for people with cardiovascular disease: Findings from the SaltSwitch randomised controlled trial. Eur J Prev Cardiol. 2017;24:1435-1444.

63. Hartman SJ, Nelson SH, Cadmus-Bertram LA, Patterson RE, Parker BA, Pierce JP. Technology- and Phone-Based Weight Loss Intervention: Pilot RCT in Women at Elevated Breast Cancer Risk. Am J Prev Med. 2016;51:714-721.

64. McCarroll ML, Armbruster S, Pohle-Krauza RJ, et al. Feasibility of a lifestyle intervention for overweight/obese endometrial and breast cancer survivors using an interactive mobile application. Gynecol Oncol. 2015;137:508-515. 
65. Mummah SA, Mathur M, King AC, Gardner CD, Sutton S. Mobile Technology for Vegetable Consumption: A Randomized Controlled Pilot Study in Overweight Adults. JMIR MHealth and UHealth. 2016;4:e51.

66. Jackson D, Turner R. Power analysis for random-effects meta-analysis. Res Synth Methods. 2017;8:290-302.

67. Charani E, Castro-Sánchez E, Moore LS, Holmes A. Do smartphone applications in healthcare require a governance and legal framework? It depends on the application! BMC Med. 2014;12:29.

68. Azar KMJ, Lesser LI, Laing BY, et al. Mobile applications for weight management: Theory-based content analysis. Am J Prev Med. 2013;45:583-589.

69. Khaylis A, Yiaslas T, Bergstrom J, Gore-Felton C. A review of efficacious technologybased weight-loss interventions: five key components. Telemed J E Health. 2010;16:931938.

70. Monica Sr. Prevention and Management of Diabetes in Norway: The Norwegian Directorate of Health; 2012.

71. Kicinski M, Springate DA, Kontopantelis E. Publication bias in meta-analyses from the Cochrane Database of Systematic Reviews. Stat Med. 2015;34:2781-2793. 

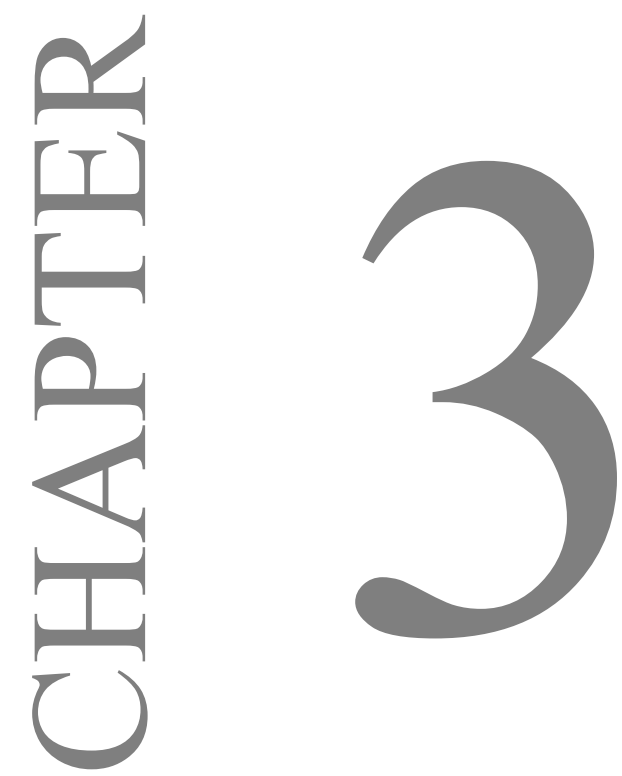

\section{DIETARY APPLICATION FOR THE MANAGEMENT OF PATIENTS WITH HEMODIALYSIS: A FORMATIVE DEVELOPMENT STUDY}

Cosette Fakih El Khoury, Mirey Karavetian, Ruud J. G. Halfens, Rik Crutzen, Dayana El Chaar, Jos M. G. A. Schols

Healthc Inform Res. 2019 October;25(4):262-273 


\section{Abstract}

Objectives: To describe the step-by-step person-centered, theory-based development of the KELA.AE app for Arabic speaking hemodialysis patients.

Methods: A step-by-step person-driven theory-based approach was conducted to develop a self-monitoring and educational dietary app for hemodialysis patients. The development follows the IDEAS (Integration, Design, Assessment, and Sharing) framework. Qualitative, semi-structured interviews with 6 hemodialysis patients and 6 healthcare practitioners (dietitians and nephrologists) were performed to assess the need for an app, the willingness to use an app and features desired in the app.

Results: KELA.AE app was developed to include a self-monitoring feature, CKD friendly recipes and a theory-based, evidence-based educational feature. Qualitative analysis of interviews revealed two predominant themes from patient interviews "Experience with the Diet", “App evaluation", and one theme from interviews with healthcare practitioners: “App evaluation". Patients expressed frustration with current accessibility to dietary information along with the need for educational materials in the app. The review of the KELA.AE prototype was positive overall, and patients reported a willingness to use the app. Healthcare practitioners considered the app accurate, simple and cultural-sensitive but expressed concerns about app misuse and replacement of healthcare practitioners.

Conclusions: KELA.AE app was found to be satisfactory and supportive to the participants' needs. Changes were made to the app as suggested during the interviews.

Keywords: Chronic Kidney Failure, Mobile Applications, Health Behavior, Diet, Telemedicine 


\section{INTRODUCTION}

A wide range of dietary mobile applications is readily available and accessible to the public $[1,2]$. Such apps are mainly used as food and exercise tracking tools [3, 4] and their usage, in this context, is effective [2,5]. Accordingly, there is a potential in digital interventions as they may reach many persons at a low cost [6].

Most available dietary apps target obesity and diabetes [1,7], and only a few tackle other medical conditions [2]. In some chronic diseases, such as chronic kidney disease (CKD), the complexity of dietary management places a burden on the patient [8]. Patients with CKD are often non-compliant to dietary restriction and particularly to phosphorous restrictions [9]. Lack of knowledge and lifestyle changes, attributed to dietary restrictions, are often the reasons for compliance challenges [9]. Accordingly, hyperphosphatemia is common among hemodialysis patients and the main characteristic of Chronic Kidney Disease-Mineral Bone Disorder (CKDMBD) [9]. Hyperphosphatemia is associated with cardiovascular disease, hyperparathyroidism, and mortality [10]. The central management of phosphorous elevations consists of dietary restrictions and the use of phosphate binders [11,12]. Therefore, management of dietary phosphorous is a particularly challenging task since it includes dietary phosphorous restrictions in parallel to adequate protein intakes [8,13]. Theory-based interventions and a problem-driven approach may facilitate adherence [14,15]. Mobile apps may provide CKD patients with continuous access to self-monitoring and nutrition education $[16,17]$. Commercial mhealth applications for CKD patients are already available on app stores [13]; however, only 50\% of available renal apps reflect the evidence-based guidelines [13].

Effective digital interventions, targeting behavioral change, should be iterative, theory-based, and tailored to the needs of the users $[18,19]$. To improve acceptability, understanding the perspective of the users, the healthcare practitioners, and the caregivers is an essential step in the development process [20]. This is described as a person-based approach [21]. The word person, rather than the user, is used as people that are not necessarily users may influence the usability [21]. Grounding in theories and literature should complement this approach [19].

Several frameworks are available to guide the development of mobile apps; to increase app usability, patient engagement in the design and development process is, therefore, essential [18-21]. The IDEAS (Integrate, Design, Assess and Share) framework suggests a comprehensive stepwise approach that includes a multidisciplinary perspective in the development of a prototype; along with grounding interventions in behavioral theory [20].

Core processes consist of a complex intervention aimed at finding answers raised at different stages within a planning framework. They are essential in the development of theory and 
evidence-based behavioral change interventions because they present a systematic and implementable way of addressing a problem [15]. Defining the problem behavior, brainstorming possible answers, and finding solutions should be included in a stepwise approach [15]. Selection and application of behavioral theories may also be tailored to the topic and the defined problem [15].

This paper aims to describe the person-centered, theory-based development of the Kidney Education for Lifestyle Application (KELA.AE app) for Arabic speaking patients. The .AE was selected as a suffix in the app name to relate the app to the internet domain name of the United Arab Emirates (UAE).

\section{MethodS}

\section{APP DEVELOPMENT}

The KELA.AE app development follows the IDEAS framework [20] to develop digital health behavior change interventions. The framework proposes a four-step approach, including Integration, Design, Assessment, and Sharing; steps are further broken down into a total of 10 sub-steps [20]. Table 1 illustrates how the development of the KELA.AE app integrates this framework. The development process was iterative, and the steps were not developed linearly. The development detailed in table 1 often required a backward flow to a previous step along with amendments and adjustments based on findings in a subsequent step. The development process extended over more than one year; it started in the fall of 2016, and the first version of the application was available in the spring of 2018. The Integrate and Design steps have been completed by the creation of the app prototype; whereas the Assess and Share steps are still in process (table 1).

\section{DEVELOPMENT OF EDUCATIONAL MATERIALS}

Previously validated stage-based Arabic educational materials targeting osteodystrophy [22] were adapted and amended to accommodate the educational modalities of the application and the Emirati Dialect. The validated materials are based on the transtheoretical model [23] and are validated both in the English and Arabic language [22]. The transtheoretical model for behavioral and readiness to change proposes a five-stage algorithm: pre-contemplation, contemplation, preparation, action, and maintenance [24]. Despite the problems that exist in the application of the stages proposed by the transtheoretical model [25], stage-based interventions have shown some promising results [24]. The validity of the stages' algorithm proposed by the transtheoretical model is not well established; accordingly, many researchers change the existing algorithm [24]. The validated stage-based materials that were used included only three different stages of change (pre-action, action, and maintenance) rather than the five 
stages proposed by the model [22]. The principal investigators performed a literature review and brainstorming sessions as part of the core processes [15]. Through this process, the authors identified a need to incorporate concepts of self-efficacy, norm, and attitudes. Accordingly, the educational materials were then translated to the mobile application features, and the narration was re-created to incorporate additional constructs from the Reasoned Action Approach [26].

The Arabic department of a national university in Dubai revised all educational materials. These were then translated back to English to ensure that the information has not been altered. All information included in educational and self-monitoring features are in line with the clinical practice guideline for the diagnosis, evaluation, prevention, and treatment of Chronic Kidney Disease-Mineral and Bone Disorder (CKD-MBD) of the Kidney Disease Improving Global Outcomes (KDIGO) [27]; thus the app content is based on evidence-based guidelines.

Three different education modalities were developed based on their reported effectiveness in the literature [28-31]. These include podcasts, videos, and notifications.

Traditional recipes were adapted to the renal diet and were also included in the application. A baseline of Arabic recipes was used from the previously validated educational materials [22], and a list of additional typical UAE recipes was collected by a group of local dietetic students from a convenience sample of four UAE nationals' households. Recipes were amended to contain less phosphorous, sodium and potassium. Local UAE households then prepared and tested the newly developed recipes and confirmed the acceptability of the recipe.

\section{INTERVIEWS, PARTICIPANTS AND DESIGN}

Qualitative, semi-structured interviews with hemodialysis patients and healthcare practitioners (dietitians and nephrologists) were performed. The aim of the interviews was to gather patients and healthcare practitioners' feedback and to involve them in the development process. the interviews with patients assessed the need for an app, the willingness to use an app and features desired in the app. Interviews with healthcare practitioners included an overview of app features along with the assessment of the need for an app by hemodialysis patients.

Patients were recruited at Al Qassimi Hospital Sharjah (UAE). Inclusion criteria consisted of: (1) adult (aged $\geq 18$ years) hemodialysis patients, (2) own and capable of operating any app on a smartphone, (3) free of life-threatening conditions, (4) on hemodialysis for three months or longer, (5) Full cognitive capacity. Healthcare practitioners recruited had to be currently practicing in a dialysis unit in the UAE. A prototype of the app, along with sample educational materials, was shown during the interviews. A sample of six patients and six healthcare practitioners were interviewed. The nature of the study was mainly formative, the scope was 
narrow, and the topic studied was rather clear [32]. Accordingly, the data gathered was homogenous and enough to provide useful information for the app development.

The study was approved by the Institutional Review Board (IRB) of Zayed University in Dubai, and informed consent was received from all participants (IRB number: MOHAP/DXB/SU BC/No-5/2016).

\section{DATA COLLECTION AND ANALYSIS}

Semi-structured interviews were conducted in Arabic, by an Emirati local dietitian, with each patient and healthcare practitioner individually. All interviews were audio-recorded and transcribed verbatim [33] and coded in Arabic using MAXQDA standard version 18.1.1 (19952018 VERBI GmbH Berlin). Interviews were performed by a moderator (an Emirati Dietitian) and an assistant facilitator (C.F.K.). The facilitator conducted the interview while the assistant recorded notes [34]. The qualitative analysis guide of Leuven (QUAGOL) was followed [35]. Stages 1 to 7 (transcription, reading, narrative reports, conceptual schemes, and coding) were performed by 2 Arabic speaking authors (C.F.K. and D.C.); whereas stages 8 to 10 (analysis of concepts and description of the results) were performed by 3 authors C.F.K., D.C. and M.K;

M.K. also coded 2 randomly selected transcripts to ensure reliability.

Table 1: Development of the KELA.AE app based on the IDEAS (Integrate, Design, Assess, Share) framework [20]

\begin{tabular}{|c|c|}
\hline \multicolumn{2}{|c|}{ Integrate insights from users and theory } \\
\hline $\begin{array}{l}\text { 1. EMPATHIZE } \\
\text { with target } \\
\text { users }\end{array}$ & $\begin{array}{l}\text { Semi-structured interviews with } 6 \text { hemodialysis patients that already own and use smartphone } \\
\text { apps. Questions were tailored around: } \\
\text { - Users' experiences with the renal diet, } \\
\text { - Their current practices } \\
\text { - Their needs and desires (for support) }\end{array}$ \\
\hline $\begin{array}{l}\text { 2. SPECIFY target } \\
\text { behavior }\end{array}$ & $\begin{array}{l}\text { Specific target behaviors were extracted based on user feedback and literature. The broad goal } \\
\text { identified is "challenges with adherence to the renal diet". Specific goals include: } \\
\text { - Restrict phosphorous, potassium, fluids and other dietary components to recommended } \\
\text { intakes } \\
\text { - Achieve target blood phosphorous, and potassium values }\end{array}$ \\
\hline $\begin{array}{l}\text { 3. GROUND in } \\
\text { behavioral } \\
\text { theory }\end{array}$ & $\begin{array}{l}\text { 1. A meta-analysis was performed to gain an understanding of the effects of the use of dietary } \\
\text { mobile applications in chronic diseases on nutritional outcomes in adults }{ }^{2} \text {. } \\
\text { 2. A multi-behavior theory approach was applied: } \\
\text { - Reasoned Action Approach [27] } \\
\text { - Transtheoretical Model [24] } \\
\text { Theories selection was based on a topic and concept approach (literature in CKD interventions } \\
\text { [28], theories used by other dietary apps [2], and concepts identified during brainstorming } \\
\text { related to self-efficacy, norms, and attitudes) }\end{array}$ \\
\hline \multicolumn{2}{|c|}{ DEsign iteratively and rapidly with user feedback } \\
\hline $\begin{array}{l}\text { 4. IDEATE } \\
\text { implementation } \\
\text { strategies }\end{array}$ & $\begin{array}{l}\text { Brainstorming sessions were held by two of the authors (C.K. and M.K.), on multiple sessions, } \\
\text { after a revision of available literature and the patients' feedback. Sessions often included } \\
\text { additional expert/s: }\end{array}$ \\
\hline
\end{tabular}




\begin{tabular}{|c|c|}
\hline & $\begin{array}{l}\text { - Local dietitian } \\
\text { - Communication expert (from the communications department at Zayed University) } \\
\text { - Arabic language expert (from the Arabic department at Zayed University) } \\
\text { - Design team } \\
\text { This process was iterative and main outputs of the sessions were decisions related to: } \\
\text { - Modes of delivery of educational materials (podcasts, videos, notifications, and recipes were } \\
\text { - developed) } \\
\text { - Content of educational materials and mapping based on theories selected } \\
\text { - Choice of nutrient databases to be included/Arabic food lists categorized as low/medium or } \\
\text { - Tigh in Phosphorous } \\
\text { - Choice of recipes to be included } \\
\text { - Order of educational messages based on a gradual building of information relayed } \\
\text { Material content was developed in line with } \mathrm{KDIGO}^{1} \text { guidelines [28]. }\end{array}$ \\
\hline $\begin{array}{l}\text { 5. PROTOTYPE } \\
\text { potential } \\
\text { products }\end{array}$ & $\begin{array}{l}\text { Several steps took place before the development of the first prototype of the app: } \\
\text { - A general wireframe (a document with sketched screenshots of the basic features of the } \\
\text { app) } \\
\text { - Detailed wireframe including screenshots of every feature in the app with design and } \\
\text { potential graphical outputs } \\
\text { Revision and adjustments of the wireframes continuously took place by C.F.K., M.K and the } \\
\text { design team and continuous changes were applied before the development of a prototype }\end{array}$ \\
\hline $\begin{array}{l}\text { 6. GATHER user } \\
\text { feedback }\end{array}$ & $\begin{array}{l}\text { Semi-structured interviews were held with patients and healthcare practitioners. The } \\
\text { interviewees were shown the app prototype and samples of the educational materials } \\
\text { - } 6 \text { hemodialysis patients that already own and use smartphone apps } \\
\text { - } 6 \text { healthcare practitioners ( } 2 \text { nephrologists and } 4 \text { dietitians) }\end{array}$ \\
\hline $\begin{array}{l}\text { 7. BUILD } \\
\text { minimum } \\
\text { viable product }\end{array}$ & $\begin{array}{l}\text { - An App prototype was developed. } \\
\text { Revision and adjustments of the prototype continuously took place by C.K., M.K and the design } \\
\text { team and continuous changes were applied before the development of the final prototype }\end{array}$ \\
\hline \multicolumn{2}{|l|}{ Assess } \\
\hline $\begin{array}{l}\begin{array}{l}\text { 8. PILOT potential } \\
\text { efficacy and } \\
\text { usability }\end{array} \\
\begin{array}{l}\text { 9. EVALUATE } \\
\text { efficacy in RCT }\end{array} \\
\end{array}$ & These two steps are in the planning step and will be reported elsewhere. \\
\hline Share & \\
\hline $\begin{array}{l}\text { 10. SHARE } \\
\text { intervention } \\
\text { and findings }\end{array}$ & $\begin{array}{l}\text { The app will be available through app stores to the public and the results of the research are } \\
\text { being and will be submitted for publication at the different steps. }\end{array}$ \\
\hline
\end{tabular}

\footnotetext{
${ }^{1}$ KDIGO: Kidney Disease Improving Global Outcomes
} 


\section{RESULTS}

\section{APP DESCRIPTION AND CONTENT}

Overall, the app was developed to contain a total of 24 podcasts, three animated videos, and 161 notifications. Videos deliver more complex concepts as compared to the other educational modalities. For example, the videos tackle concepts such as the phosphorus to protein ratio of food, which guides patients to identify and consume foods that are rich in proteins yet low in phosphorous. Tables 2,3 , and figure 1 depict some examples of stagebased podcasts, videos, and notifications.

Additionally, the app includes 26 recipes along with their nutrient analysis for salt, potassium, phosphorus, and protein content. Furthermore, the users can access selfmonitoring features, consisting of a food intake diary and a laboratory values tracker. Sample screenshots from the app are presented in Figure 2.

The app name was selected as KELA.AE; "KELA" stands for Kidney Education for Lifestyle Application, but KELA also means kidney in the Arabic Language. The app design is culturespecific, in terms of dialect, recipes, food selections, and graphics (for example, characters represent male and female Emirati patients).

Table 2: Sample stage-based podcasts matched to constructs of the Reasoned Action Approach

\begin{tabular}{|c|c|c|}
\hline $\begin{array}{l}\text { Stage of } \\
\text { change }\end{array}$ & $\begin{array}{l}\text { Construct from } \\
\text { Reasoned Action } \\
\text { Approach }\end{array}$ & Example of Podcast script \\
\hline Pre-Action & $\begin{array}{l}\text { Norm, } \\
\text { Capacity/Self- } \\
\text { efficacy }\end{array}$ & $\begin{array}{l}\text { We are going to talk about how small changes can positively } \\
\text { affect the quality of your life. } \\
\text { By following a diet specific to dialysis, exercising, and taking } \\
\text { specific medications, you can improve your lab tests. Your lab } \\
\text { tests are affected by your diet, for example if your diet is high in } \\
\text { the mineral phosphorus, then your phosphorus level in the blood } \\
\text { will also rise and that is not something desired for your health. } \\
\text { I know people, on dialysis, who suffered from itching and sleep } \\
\text { disturbances; the reason for these symptoms was unclear to } \\
\text { them, but by taking care of their diet and lowering the intake of } \\
\text { foods high in the mineral phosphorus, the itching and sleep } \\
\text { disturbances improved, and they felt better. You have the power } \\
\text { to manage your own diet, and you will see improvement in your } \\
\text { life and reduce the risks to your health as soon as you make small } \\
\text { changes. }\end{array}$ \\
\hline Action & Attitude & $\begin{array}{l}\text { Being on a low phosphorus diet is important to prevent a high } \\
\text { blood phosphorus level, and therefore prevent many } \\
\text { complications like bone or heart diseases. } \\
\text { Phosphorus is present in many foods especially those rich in } \\
\text { protein. That doesn't mean you must cut down on proteins } \\
\text { because your body needs protein for optimal functioning. The }\end{array}$ \\
\hline
\end{tabular}




\begin{tabular}{ll}
\hline & $\begin{array}{l}\text { solution is to consume proteins that are as low in Phosphorous as } \\
\text { possible. } \\
\text { Some examples of high protein foods but low or moderate P are } \\
\text { Fish, Poultry, Lean meats, Crab and Shrimps }\end{array}$ \\
\hline Maintenance Capacity/Self- & $\begin{array}{l}\text { Make physical activity a habit. This way it will become easier to } \\
\text { comply with. Have fun while exercising, invite your friends, family } \\
\text { of neighbors to join you. } \\
\text { Make an agenda for physical activity to remind you to exercise! } \\
\text { After complying for a while, you will feel very rewarded and proud }\end{array}$ \\
\hline
\end{tabular}

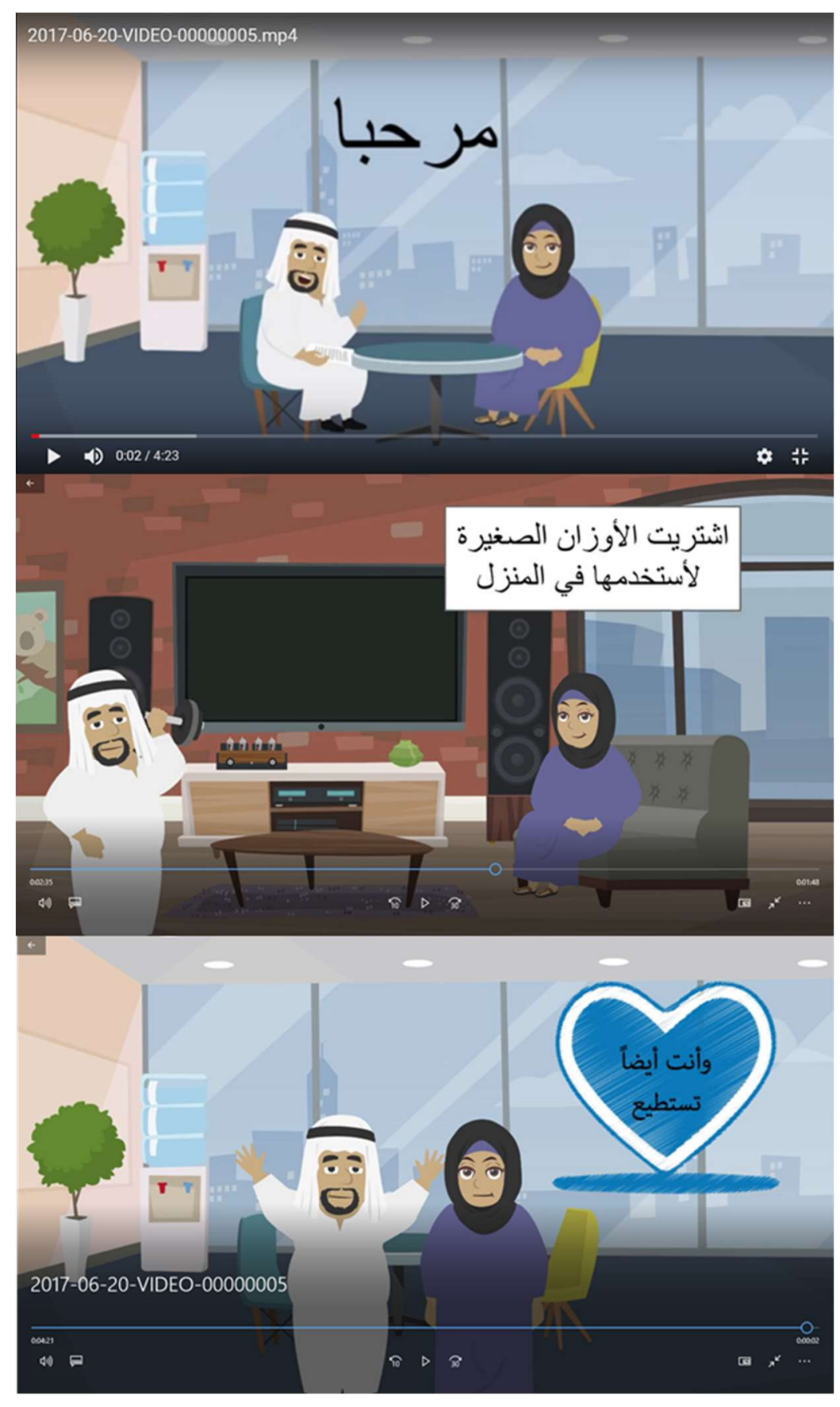

Figure 1: Sample Video snapshot 


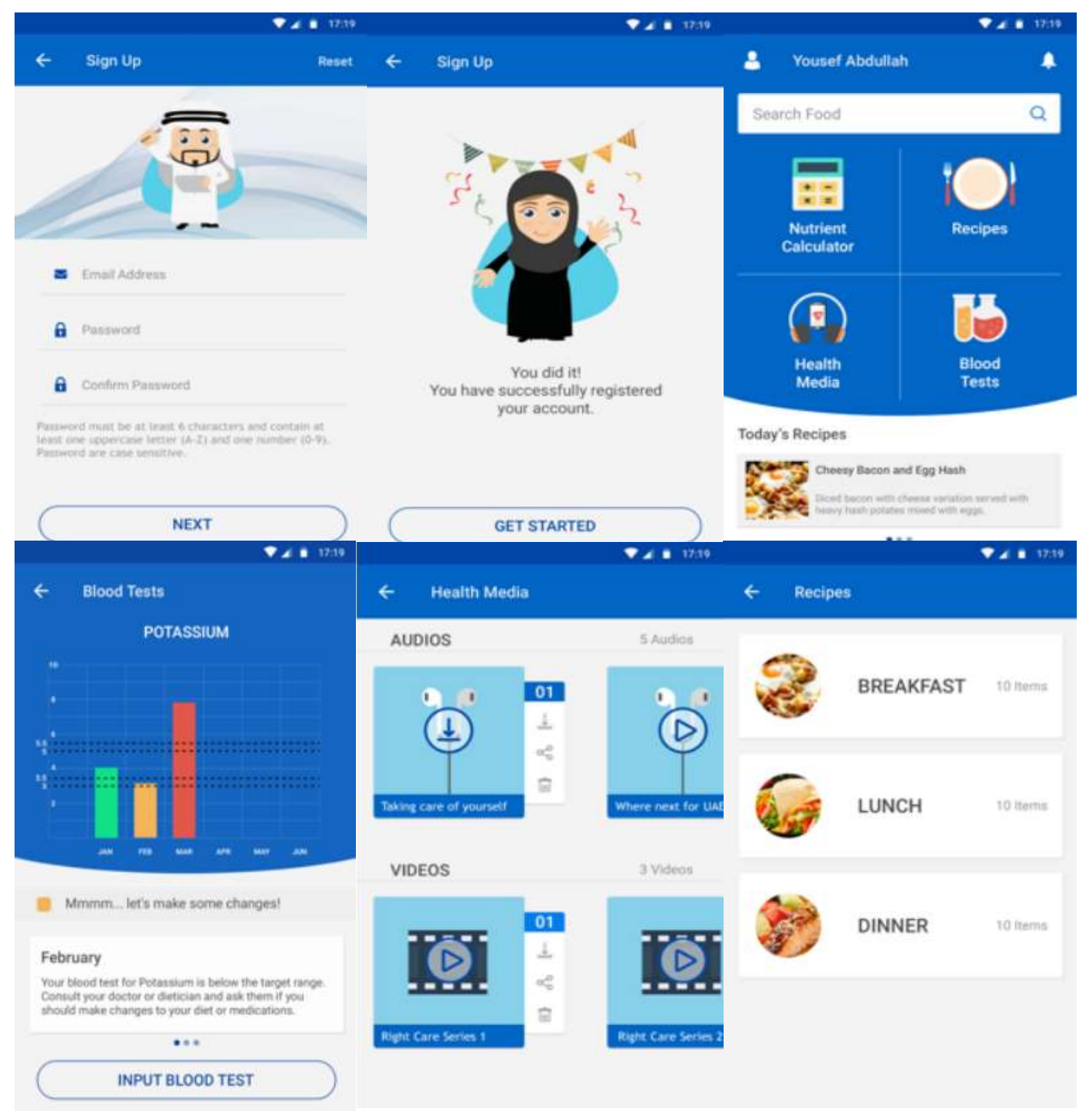

Figure 2: Sample screenshots from the KELA.AE app

\section{KEY FINDINGS FROM PATIENTS INTERVIEWS}

A total of six patients were interviewed, four males and two females. The mean age of the patients was 47 years (SD 15, Range 37); all patients were of Arab nationalities (four UAE nationals, one Omani and one Lebanese) and have been on dialysis for more than three months. Though computer literacy levels were not formally measured during development, inclusion criteria addressed the capacity to operate a mobile app. The interview was started by asking patients about their experience with the renal diet in general. Patients were then shown the app prototype and the educational materials and asked for feedback. Figure 3 summarizes the key findings from the patients' interviews. 


\section{Experience with the Diet}

Frustration concerning access to information was common among patients; many expressed struggles with finding reliable information on the hemodialysis diet. Although dietitians are available at the dialysis center, patients desire continuous access to information concerning food selections. Patients reported using the internet as the most accessible and most convenient platform for answers regarding the diet, despite knowing that it may lack some accuracy. All patients, except one, reported referring directly to their physicians.

They also described the experience pertinent to the renal diet to be more challenging at the start of dialysis but gets more comfortable with time; and expressed that sharing information with other dialysis patients facilitated the learning process. Most patients expressed a need for educational features and constant access to useful information through an app, rather than self-monitoring features.

"At the beginning, I was very lost, then I started asking here and there (to understand the diet better), I would see other dialysis patients, and they would talk about certain things, and I would ask, and slowly, slowly I started understanding more" (MP2/71-73).

\section{App evaluation}

The patients were shown an app prototype, along with samples of the educational materials. Most of them showed interest in educational materials mainly. Patients expressed preferring the podcasts and videos over traditional paper-based education.

When asked how easy they found the app, on a scale of 1 to 10 , they answered 7/10 as the lowest score, with a median score of 8/10. Some defined the app as "easy" (FP1/12), or "very useful" (MP1/24); while one patient was hesitant and preferred being able to use it before providing feedback.

"I would have to access the app and use it to be able to give you any feedback" (MP4/69-70).

Patients were asked to provide possible suggestions or changes to the app during the interview. Two leading recommendations were provided, including a search feature to determine the nutrient content of cultural foods and to have access to the dietitian through the app.

"For example, can the dietitian be on the app so she can send us things?" (MP4/75-76). 


\section{Key Findings from Patients}

\section{Theme: Experience with Diet}

- Sub-theme: Current Status

- Frustration pertinent lack of access to continuous information

- Constant challenges particularly at the beginning

- Sub-theme: Need for education

- Continuous access

- Education of food selections

- Role of other patients

\section{Theme: App Evaluation}

\section{-Sub-theme: Current Features}

- Preference for educational features

- How easy is the app on a scale of 1 to 10? Median score of 8/10

- Sub-theme: Additional Features/ Suggestions

- Search Feature for nutrient compositionof cultural foods

-In-app access to the Dietitian

Figure 3: Key Findings from patients interviews on the KELA.AE app

\section{KEY FINDINGS FROM HEALTHCARE PRACTITIONERS INTERVIEWS}

A total of six healthcare practitioners were interviewed, consisting of two Nephrologists and four Dietitians. Healthcare practitioners were shown an overview of all the features of the app, and then they were asked to provide feedback. Figure 4 summarizes the key findings from the healthcare practitioners' interviews.

\section{App Evaluation}

Overall, healthcare practitioners found the app accurate, simple, and culturally sensitive; the features of the app were considered comprehensive, organized, and tailored to patients' needs. Positive remarks provided mainly addressed the videos and podcasts, as well as the overall design and graphics of the logo and the application.

One Nephrologist said: "My feedback is very positive, I like this application, it is very informative, and it will help our patients a lot. Specifically, when it is in both languages, English and Arabic. It is a very positive tool in the management of the patients; because the diet in dialysis patients is a very important aspect of any stage of renal disease patient, and without this, it's very harsh to manage our patients, without this diet component" (NH1/45). 
However, some concerns emerged regarding app misuse. One nephrologist expressed fear that patients may exchange information with other dialysis patients to whom such dietary guidelines may not apply.

For instance, one Nephrologist said: "if I told one patient that is not diabetic and does not have hypertension if he told his friend which is diabetic and hypertensive, he would take this one and he will... it's not good." (NH2/94).

An additional concern was also discussed, and that is related to the fear of the app replacing the healthcare practitioner.

"I would not strongly advice that they should self-estimate their potassium and phosphorus levels every time, they can have an idea, but we would like to advise them to see or consult us that this was our readings in the application so that we can review, or our dialysis dietitian can review them. They can have an idea, but not I will not recommend that they solely rely on that" (NH1/51-52).

\section{Suggestions}

Healthcare practitioners, including one nephrologist (NH2) and one dietitian (DH1), suggested adding more features, mainly to make the app more comprehensive in terms of coverage for education on potassium, sodium, fluids as well as diabetes, dyslipidemia, and hypertension management. One Nephrologist said: "I would like the recipes to specify if they are suitable for patients with diabetes or hypertension...90\% of dialysis patients suffer from diabetes or hypertension" (NH2/14). Two dietitians suggested to include more educational material on protein sources and quantities (DH1 and DH4). Healthcare practitioners (NH2 and DH1) also focused on the need for more information about fluid intake and its management. For example, one dietitian said: "I would suggest adding this part (referring to water), so they can have a part for calculation of such issues (referring to tracking and selfmanagement of fluids)" (DH4/43). Suggestions for more educational material covering additional minerals, such as iron, were made by one nephrologist and dietitian (NH2 and DH1). 


\section{Key Findings from Healthcare Professionals}

\section{Theme: App Evaluation}

-Sub-theme: Current Features

-Accurate, simple and culturally sensitive

- Concern with app misuse and replacement of healthcare practitioners

-Sub-theme: Additional Features/ Suggestions

- More educational features targeting issues other than CKD-MBD (potassium, diabetes, HTN, fluid management, protein intake, iron..)

Figure 4: Key Findings from Healthcare Professionals on the KELA.AE app

\section{DISCUSSION}

The KELA.AE app was developed based on a person-centered, theory-based approach. The app includes self-monitoring and educational features. Previous literature has shown that mobile applications may serve as effective self-monitoring tools [36], and they have the potential to deliver education [13] to patients. Tailoring the app content to patient needs and incorporating behavioral theories are criteria that contribute to well-designed dietary apps [37].

The patients interviewed during the development reported internet usage as a nutrition information source. Such practices have grown in the past years, as many rely on the internet as an information source [38]. A lot of nutritional information is available on the renal diet through websites and YouTube videos [39]. Content analysis showed that most of the information found on websites is evidence-based; however, it is not easily understandable and actionable; whereas information on YouTube tended to be less evidence-based yet more understandable [39]. Accordingly, there seems to be a need for information developed based on evidence, addressing the health literacy of renal patients [40].

The results of the interviews with patients emphasized a need for education rather than selfmonitoring. These findings have also been described by a recent systematic review, reporting that most available CKD apps contain food dairies and dietary recommendations, whereas CKD-friendly recipes and in-app education were not as common. The review also addressed the need for in-app education to cater for different learning styles [41]. The patients in this study also expressed the need for several educational features; hence, we incorporated recipes and used different education modalities in the KELA.AE app, based on data gathered from interviews and recent literature.

The perspective of other persons that may influence app usage is also essential $[42,43]$. Thus, healthcare practitioners were also interviewed and shown an app prototype. Generally, they 
evaluated the app positively; however, some raised concerns related to app misuse and replacement of the healthcare practitioner.

Digital interventions may support a reciprocal relationship between patient and healthcare practitioners and enhance patient-centered care [44]. However, the extent to which a healthcare practitioner is willing to engage in a collaborative partnership with the patient might play a role in the effect of an eHealth intervention [45]. Additionally, the perceived quality of the physician (defined as perceived physician knowledge and communication skills), and the quality of the information (defined as adequacy, relevance, and usefulness of the information) retrieved by patients online have a significant impact on the patient-physician relationship [46].

Some challenges were faced that were mainly related to technical matters. However, the main limitation was incorporating a nutrient database into the self-monitoring feature of the app. Using nutrient data sources developed in different countries may lead to significant errors in the assessment of nutrient composition [47]. Nevertheless, this issue is not only applicable to apps, as practitioners in the Arab world commonly use foreign nutrient databases due to the lack of a reliable Arabic database. Additionally, the database is in the English language, which is the main barrier to the Arabic speaking user. Based on patient suggestions, a list of Arabic foods categorized by their mineral content was made available on the app to compensate for this limitation.

The strength of this study lies in the involvement of the patients and healthcare practitioners in app development. Patients have previously expressed the request to collaborate on the development of solutions tailored to support them in self-management [48]. KELA.AE development involved patients and healthcare practitioners in a structured approach and collected qualitative data that guided the app development. Given the nature of the study, data gathered was informative and therefore a larger sample size was not required.

Future research should address the effectiveness of CKD apps, as educational tools, in increasing patient knowledge and adherence to the renal diet.

The KELA.AE app was developed to close the current gap between the patients' need for information while incorporating behavioral-theories and using an iterative, person-driven approach. We consider this development method supportive for app developers as it is replicable across different cultures, languages, and chronic diseases. Overall, the app review was positive, and patients expressed willingness to use the app, while healthcare practitioners considered it useful. Feasibility, usability, and effectiveness of the app will be assessed through an interventional trial at a later stage. 


\section{ACKNOWLEDGMENTS}

We acknowledge the staff and students from Zayed University who volunteered by contributing to the app development at many steps 


\section{REFERENCES}

1. Martínez-Pérez B, De La Torre-Díez I, López-Coronado M. Mobile health applications for the most prevalent conditions by the World Health Organization: review and analysis. Journal of medical Internet research. 2013;15(6):e120.

2. El Khoury CF, Karavetian M, Halfens RJ, Crutzen R, Khoja L, Schols JM. The Effects of Dietary Mobile Apps on Nutritional Outcomes in Adults with Chronic Diseases: A Systematic Review and Meta-Analysis. Journal of the Academy of Nutrition and Dietetics. 2019;119(4):626-51.

3. DiFilippo KN, Huang W-H, Andrade JE, Chapman-Novakofski KM. The use of mobile apps to improve nutrition outcomes: a systematic literature review. Journal of telemedicine and telecare. 2015;21(5):243-53.

4. Boyce B. Nutrition apps: opportunities to guide patients and grow your career. Journal of the Academy of Nutrition and Dietetics. 2014;114(1):13-4.

5. Carter MC, Burley V, Nykjaer C, Cade J. 'My Meal Mate'(MMM): validation of the diet measures captured on a smartphone application to facilitate weight loss. British Journal of Nutrition. 2013;109(3):539-46.

6. Bennett GG, Glasgow RE. The delivery of public health interventions via the Internet: actualizing their potential. Annual review of public health. 2009;30:273-92.

7. Turner-McGrievy GM, Beets MW, Moore JB, Kaczynski AT, Barr-Anderson DJ, Tate DF. Comparison of traditional versus mobile app self-monitoring of physical activity and dietary intake among overweight adults participating in an mHealth weight loss program. Journal of the American Medical Informatics Association. 2013;20(3):513-8.

8. D'Alessandro C, Piccoli GB, Cupisti A. The "phosphorus pyramid": a visual tool for dietary phosphate management in dialysis and CKD patients. BMC nephrology. 2015;16(1):9.

9. Durose CL, Holdsworth M, Watson V, Przygrodzka F. Knowledge of dietary restrictions and the medical consequences of noncompliance by patients on hemodialysis are not predictive of dietary compliance. Journal of the American Dietetic Association. 2004;104(1):35-41.

10. Abe M, Okada K, Soma M. Mineral metabolic abnormalities and mortality in dialysis patients. Nutrients. 2013;5(3):1002-23.

11. Melamed ML, Buttar RS, Coco M. CKD-mineral bone disorder in stage 4 and 5 CKD: what we know today? Advances in chronic kidney disease. 2016;23(4):262-9.

12. Newsome B, Ix JH, Tighiouart H, Sarnak MJ, Levey AS, Beck GJ, et al. Effect of protein restriction on serum and urine phosphate in the modification of diet in renal disease (MDRD) study. American Journal of Kidney Diseases. 2013;61(6):1045-6. 
13. Lambert K, Mullan J, Mansfield K, Owen P. Should We Recommend Renal DietRelated Apps to Our Patients? An Evaluation of the Quality and Health Literacy Demand of Renal Diet-Related Mobile Applications. Journal of Renal Nutrition. 2017;27(6):4308 .

14. Clark S, Farrington K, Chilcot J, editors. Nonadherence in dialysis patients: prevalence, measurement, outcome, and psychological determinants. Seminars in dialysis; 2014: Wiley Online Library.

15. Ruiter RA, Crutzen R, Kok G. Core Processes for Developing Theory-and EvidenceBased Interventions. 2018.

16. Prest M. Mobile Phone Applications for Kidney Patients. Journal of Renal Nutrition. 2013;23(4):e83-e5.

17. Hutchesson MJ, Rollo ME, Callister R, Collins CE. Self-monitoring of dietary intake by young women: online food records completed on computer or smartphone are as accurate as paper-based food records but more acceptable. Journal of the Academy of Nutrition and Dietetics. 2015;115(1):87-94.

18. Schnall R, Rojas M, Bakken S, Brown W, Carballo-Dieguez A, Carry M, et al. A usercentered model for designing consumer mobile health (mHealth) applications (apps). Journal of biomedical informatics. 2016;60:243-51.

19. Brown III W, Yen P-Y, Rojas M, Schnall R. Assessment of the Health IT Usability Evaluation Model (Health-ITUEM) for evaluating mobile health (mHealth) technology. Journal of biomedical informatics. 2013;46(6):1080-7.

20. Mummah SA, Robinson TN, King AC, Gardner CD, Sutton S. IDEAS (Integrate, Design, Assess, and Share): a framework and toolkit of strategies for the development of more effective digital interventions to change health behavior. Journal of medical Internet research. 2016;18(12).

21. Birnbaum F, Lewis DM, Rosen R, Ranney ML. Patient engagement and the design of digital health. Academic emergency medicine: official journal of the Society for Academic Emergency Medicine. 2015;22(6):754.

22. Karavetian M, de Vries N, Elzein H, Rizk R, Bechwaty F. Effect of behavioral stagebased nutrition education on management of osteodystrophy among hemodialysis patients, Lebanon. Patient education and counseling. 2015;98(9):1116-22.

23. Prochaska JO, Velicer WF. The transtheoretical model of health behavior change. American journal of health promotion. 1997;12(1):38-48.

24. Brug J, Conner M, Harre N, Kremers S, McKellar S, Whitelaw S. The Transtheoretical Model and stages of change: a critique: observations by five commentators on the paper by Adams, J. and White, M.(2004) why don't stage-based activity promotion interventions work? Health education research. 2004;20(2):244-58. 
25. Adams J, White M. Why don't stage-based activity promotion interventions work? Health education research. 2004;20(2):237-43.

26. Fishbein M. A reasoned action approach to health promotion. Medical Decision Making. 2008;28(6):834-44.

27. Group KDIGOC-MW. KDIGO clinical practice guideline for the diagnosis, evaluation, prevention, and treatment of Chronic Kidney Disease-Mineral and Bone Disorder (CKDMBD). Kidney international Supplement. 2009(113):S1.

28. Turner-McGrievy G, Tate D. Tweets, Apps, and Pods: Results of the 6-month Mobile Pounds Off Digitally (Mobile POD) randomized weight-loss intervention among adults. Journal of medical Internet research. 2011;13(4):e120.

29. Tuong W, Larsen ER, Armstrong AW. Videos to influence: a systematic review of effectiveness of video-based education in modifying health behaviors. Journal of behavioral medicine. 2014;37(2):218-33.

30. Haapala I, Barengo NC, Biggs S, Surakka L, Manninen P. Weight loss by mobile phone: a 1-year effectiveness study. Public health nutrition. 2009;12(12):2382-91.

31. Kerr DA, Pollard CM, Howat P, Delp EJ, Pickering M, Kerr KR, et al. Connecting Health and Technology (CHAT): protocol of a randomized controlled trial to improve nutrition behaviours using mobile devices and tailored text messaging in young adults. BMC public health. 2012;12(1):477.

32. Morse JM. Determining sample size. Sage Publications Sage CA: Thousand Oaks, CA; 2000.

33. Bailey J. First steps in qualitative data analysis: transcribing. Family practice. 2008;25(2):127-31.

34. Winslow WW, Honein G, Elzubeir MA. Seeking Emirati women's voices: The use of focus groups with an Arab population. Qualitative Health Research. 2002;12(4):566-75.

35. de Casterle BD, Gastmans C, Bryon E, Denier Y. QUAGOL: A guide for qualitative data analysis. International journal of nursing studies. 2012;49(3):360-71.

36. Tanenbaum ML, Bhatt HB, Thomas VA, Wing RR. Use of self-monitoring tools in a clinic sample of adults with type 2 diabetes. Translational behavioral medicine. 2016;7(2):358-63.

37. Azar KM, Lesser LI, Laing BY, Stephens J, Aurora MS, Burke LE, et al. Mobile applications for weight management: theory-based content analysis. American journal of preventive medicine. 2013;45(5):583-9. 
38. Pollard CM, Pulker CE, Meng X, Kerr DA, Scott JA. Who uses the Internet as a source of nutrition and dietary information? An Australian population perspective. Journal of medical Internet research. 2015;17(8).

39. Lambert K, Mullan J, Mansfield K, Koukomous A, Mesiti L. Evaluation of the quality and health literacy demand of online renal diet information. J Hum Nutr Diet. 2017;30(5):634-45. Epub 2017/02/18. doi: 10.1111/jhn.12466. PubMed PMID: 28211108 .

40. Fraser SD, Roderick PJ, Casey M, Taal MW, Yuen HM, Nutbeam D. Prevalence and associations of limited health literacy in chronic kidney disease: a systematic review. Nephrology Dialysis Transplantation. 2012;28(1):129-37.

41. Kosa SD, Monize J, D'Souza M, Joshi A, Philip K, Reza S, et al. Nutritional Mobile Applications for CKD Patients: Systematic Review. Kidney Int Rep. 2019;4(3):399-407. Epub 2019/03/23. doi: 10.1016/j.ekir.2018.11.016. PubMed PMID: 30899867; PubMed Central PMCID: PMCPMC6409338.

42. Ong SW, Jassal SV, Porter E, Logan AG, Miller JA. Using an electronic selfmanagement tool to support patients with chronic kidney disease (CKD): a CKD clinic self-care model. Semin Dial. 2013;26(2):195-202. Epub 2013/02/15. doi:

10.1111/sdi.12054. PubMed PMID: 23406283.

43. Damodaran L. User involvement in the systems design process-a practical guide for users. Behaviour \& information technology. 1996;15(6):363-77.

44. Macdonald GG, Townsend AF, Adam P, Li LC, Kerr S, McDonald M, et al. eHealth technologies, multimorbidity, and the office visit: qualitative interview study on the perspectives of physicians and nurses. Journal of medical Internet research. 2018;20(1).

45. Ammenwerth E. From eHealth to ePatient: The Role of Patient Portals in Fostering Patient Empowerment. EJBI. 2018;14(2):20-3.

46. Laugesen J, Hassanein K, Yuan Y. The impact of internet health information on patient compliance: a research model and an empirical study. Journal of medical Internet research. 2015;17(6).

47. Cashel K, Greenfield H. The effects of Australian, US and UK food composition tables on estimates of food and nutrient availability in Australia. 1995.

48. Rogers D. Patient Perspective of Smartphone-Based Apps for CKD Self-Care. Clin J Am Soc Nephrol. 2019;14(4):483-4. Epub 2019/03/23. doi: 10.2215/CJN.02220219. PubMed PMID: 30898875 ; PubMed Central PMCID: PMCPMC6450355. 

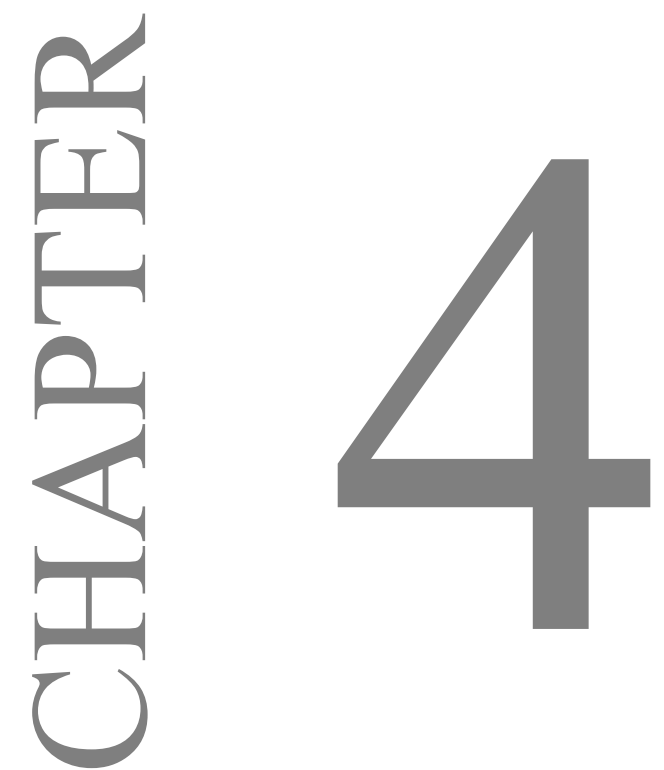

\section{A DIETARY MOBILE APP FOR HEMODIALYSIS PATIENTS: A PILOT STUDY}

Cosette Fakih El Khoury, Rik Crutzen, Jos M.G.A. Schols, Ruud J.G. Halfens and Mirey Karavetian

JMIR Preprints. 14/01/2020:17817 (submitted) 


\section{Abstract}

\section{Abstract}

Background: Mobile technology has an impact on the healthcare sector, also within dietetics. Mobile health applications may be used for dietary assessment and self-monitoring, allowing for real-time reporting of food intakes. Changing eating behaviors is quite challenging, and hemodialysis patients, particularly, struggle to meet the target intakes set by dietary guidelines. Usage of mobile apps that are developed in a person-centered approach and that are in line with recommendations may support both patients and healthcare practitioners.

Objective: This study is a pilot that aims at estimating the potential efficacy of a dietary intervention using a theory-based, person-centered smartphone app. Results will be used to improve both the application and a planned large-scale trial intended to assess app efficacy thoroughly.

Methods: A prospective pilot study was performed at the hemodialysis unit of Al Qassimi Hospital (Emirate of Sharjah). All patients that fulfilled the study inclusion criteria were considered eligible to be enrolled in the pilot study. Upon successful installation of the app, users met with a dietitian once a week. Outcomes were measured at baseline (T0) and two weeks post app usage (T1). This pilot is reported as per guidelines for non-randomized pilot and feasibility studies and in line with the CONSORT 2010 checklist for reporting pilot or feasibility trials.

Results: Twenty-three subjects completed the pilot intervention. Mean energy intakes increased from $24.4 \mathrm{Kcal} / \mathrm{kg} / \mathrm{d}(8.0)$ to $29.1 \mathrm{Kcal} / \mathrm{kg} / \mathrm{d}$ (7.8) with a medium effect size ( $d=0.6$; $95 \%$ CI $0.0-1.2)$. Mean protein intakes increased from $0.9 \mathrm{~g} / \mathrm{kg} / \mathrm{d}(0.3)$ to $1.3 \mathrm{~g} / \mathrm{kg} / \mathrm{d}(0.5)$ with a large effect size ( $d=1.0 ; 95 \% \mathrm{CI} 0.4-1.6)$; intake of high biological value proteins (\%HBV) also increased from $58.6 \%(10.1)$ to $70.1 \%$ (10.7) with a large effect size $(d=1.1 ; 95 \%$ CI $0.5-1.7)$. Dietary intakes of minerals did not change, apart from sodium which decreased from an intake of $2218.8 \mathrm{mg} / \mathrm{d}(631.6)$ to $1895.3 \mathrm{mg} / \mathrm{d}(581.0)$ with a medium effect size $(d=0.5 ; 95 \% \mathrm{CI} 0.1$ 1.1). Serum phosphorus, potassium and albumin did not change relevantly. Serum iron increased from $7.9 \mathrm{mg} / \mathrm{dl}(2.8)$ to $11.5 \mathrm{mg} / \mathrm{dl}(7.9)$ post-intervention with a medium effect size $(d=0.6 ; 95 \%$ CI $0.0-1.2)$.

Conclusions: This pilot study showed that KELA.AE app has the potential to improve dietary intakes. Processes related to procedure, resources, tools and app improvement for a future trial were assessed. A more extended intervention using a randomized controlled trial is required to estimate parameters concerning app efficacy accurately. 
Keywords: mobile health, dietary app, hemodialysis diet

\section{INTRODUCTION}

Mobile technology has the potential to improve healthcare coverage, especially in low to middle-income countries where people may be more likely to have access to a smartphone than to basic needs such as safe water and electricity [1]. Self-tracking and wearable technologies have become popular, particularly in the areas of diet and fitness [2]. Previous research on mobile health interventions provides evidence regarding their effectiveness [3-5]. However, their role as educational tools or as supportive tools to standard care is still inconclusive $[3,6]$.

Mobile technology may assist in the introduction of new methods of dietary assessment and self-monitoring, allowing for real-time reporting of food intakes [7]. Self-monitoring is an important factor in successful dietary behavior changes [8]. In a previous study on a weight loss intervention, the more participants recorded food intakes on a mobile app, the more likely they were to lose weight [9]. However, patients may not always be interested and willing to track intakes [10]; and commitment to self-monitoring decreases over time, even with the use of mobile technology [11].

Changing eating behaviors is difficult [12], and adherence to guidelines is challenging, especially for patients with chronic conditions such as chronic kidney disease (CKD) [13]. Hemodialysis patients, particularly, struggle to meet the target intakes set by dietary guidelines [14]. The diet during hemodialysis is somewhat restrictive, requiring the management of potassium, phosphorus, sodium, and fluids while maintaining adequate protein and energy intakes to prevent malnutrition [15]. In a recent study assessing adherence to diet guidelines among hemodialysis patients, $77 \%$ and $50 \%$ of the patients respectively consumed less energy and proteins than recommended. These low intakes could be attributed to the restrictive nature of the dialysis diet. In the same study, participants were also found to consume excessive saturated fats and inadequate intakes of fibers and micronutrients [14]. Maintaining adequate intake of minerals such as potassium, phosphorus, and calcium is essential in the prevention of dialysis-related complications such as heart failure, metabolic bone disorders, and mortality $[16,17]$. Protein-energy malnutrition is also a contributor to complications, and malnutrition among dialysis patients is a predictor of mortality [18]. Accordingly, it seems that the diet quality of hemodialysis patients is often poor and that they do not follow dialysis specific guidelines $[14,19]$. 
The main challenge in changing dietary behavior is developing interventions that are comprehensive and sustainable, promoting long-term changes in eating habits and lifestyle [12]. There is a need for nephrologists and dietitians to adopt approaches that strengthen educational and clinical interventions [14]. Possibly, mobile technology could be used to enhance dietetic practice by providing support to patients and dietitians. Thus, adding mobile apps to in-person counseling may provide more accessible and flexible dietetic services at lower costs [20]. However, research on the role of mHealth on dietary behavior in hemodialysis patients is still scarce [21].

This study is a pilot that aims at estimating the potential of a dietary intervention using a smartphone app in hemodialysis patients. The results from this pilot study will be used to improve the app itself as well as the study design of the planned randomized controlled trial.

\section{METHODS}

\section{PARTICIPANTS}

All patients at the hemodialysis unit of Al Qassimi Hospital (Emirate of Sharjah) who fulfilled the study inclusion criteria were considered eligible to be enrolled in the pilot study. Inclusion criteria comprised patients on hemodialysis for at least three months, free of life-threatening conditions, able to read, write, listen and communicate, owning an Android smartphone, and not having been hospitalized in the past six months. All subjects approached who accepted to enroll in the study signed written consent forms. A total of 26 participants were enrolled in the pilot, of which 23 downloaded and used the app for two weeks. Data were collected between February 2019 and April 2019. Post-hoc calculations of sample size in pilot studies, assuming detection of unanticipated problems with a probability of at least $15 \%$ probability $(\pi=0.15)$ and a $95 \%$ confidence level, resulted in a required sample of 19 subjects [22].

\section{KELA.AE APP}

KELA.AE (Kidney Education for Lifestyle Application) is an Arabic, culturally specific, educational, and self-monitoring app that was developed in a person-centered, theory-based approach. Educational materials were developed based on the Transtheoretical Model [23] and constructs of the Reasoned Action Approach [24]. Included educational materials are podcasts, videos, notifications, and recipes. The app also provides self-monitoring features that allow patients to track food intakes and blood parameters. The app was developed following the IDEAS (Integrate, Design, Assess, and Share) Framework [25]; a detailed description of the 
app development is described elsewhere [10]. Figure 1 depicts screenshots from the application.
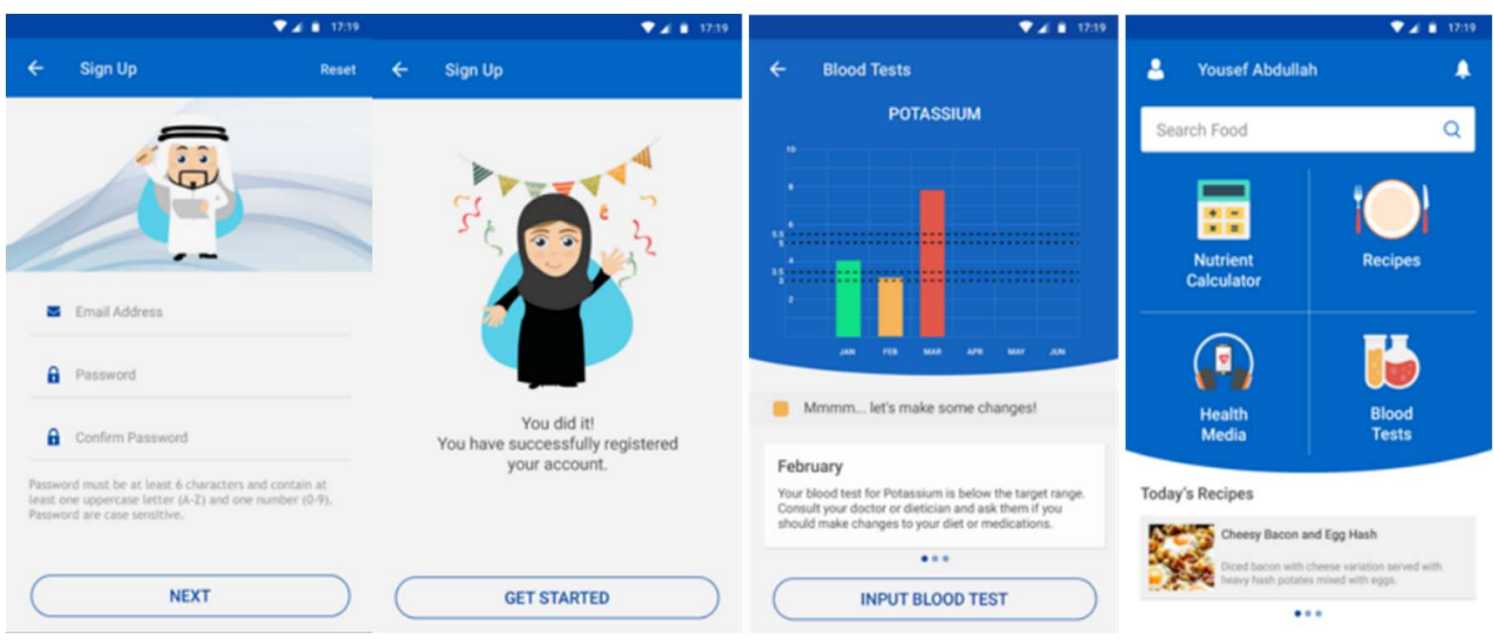

Figure 5: Screenshots of the KELA.AE app used during the pilot study

\section{PROCEDURE}

This pilot is reported as per guidelines for non-randomized pilot and feasibility studies [26] and in line with the CONSORT 2010 checklist for reporting a pilot or feasibility trial (items pertinent to randomization were considered not applicable) [27]. Ethical approval was received from the Institutional Review Board (IRB) of Zayed University, Dubai (Ethical Approval number ZU17_066_F). A research grant by Zayed University, Dubai funded the study (Research Grant number R18060).

Participants who consented to enroll in the pilot study downloaded the app and were provided with usernames that allowed them to sign-in to the app. Upon successful installation of the app, users met with a dietitian once a week (twice in total); the dietitians responded to concerns pertinent to app usage, but also questions about the renal diet by reinforcing the critical messages relayed by the educational materials. The dietitians also collected the data from patients before and after app usage. All outcomes were measured before (T0) and after two weeks (T1) of app usage.

\section{DIETARY INTAKES}

Face-to-face 24-hour recalls were collected [28] at T0 and T1 for all participants; nutrient compositions were derived for energy, carbohydrates, proteins, high biological value proteins, total fat, potassium, phosphorus, and sodium. Intakes were then compared to dietary 
guidelines for hemodialysis patients [15,29]. Protein targets were considered as $\geq 1.2 \mathrm{~g} / \mathrm{Kg}$ with $\geq 50 \%$ high biological value (HBV) protein; energy as $30-35 \mathrm{Kcal} / \mathrm{Kg}$, Phosphorus as $1000 \mathrm{mg} / \mathrm{d}$ for participants with serum phosphorus below $5.5 \mathrm{mg} / \mathrm{d}$ and $12 \mathrm{mg}$ per gram of protein intake for participants with serum phosphorus below $5.5 \mathrm{mg} / \mathrm{dl}$. Sodium and Potassium targets were considered as less than 2400mg/d [15,29]. Standard body weight (SBW) from the National Health and Nutrition Examination Study was used for calculations; however, adjusted edema-free body weight (aBWef) was used for calculating nutrient needs for individuals with $<95 \%$ or $>115 \%$ of SBW [29]. Textbox 1 illustrates the dietary guidelines that were used as targets and that are also included in the adherence index.

Textbox 1. Dietary guidelines for patients on hemodialysis used for adherence index ${ }^{15,29}$

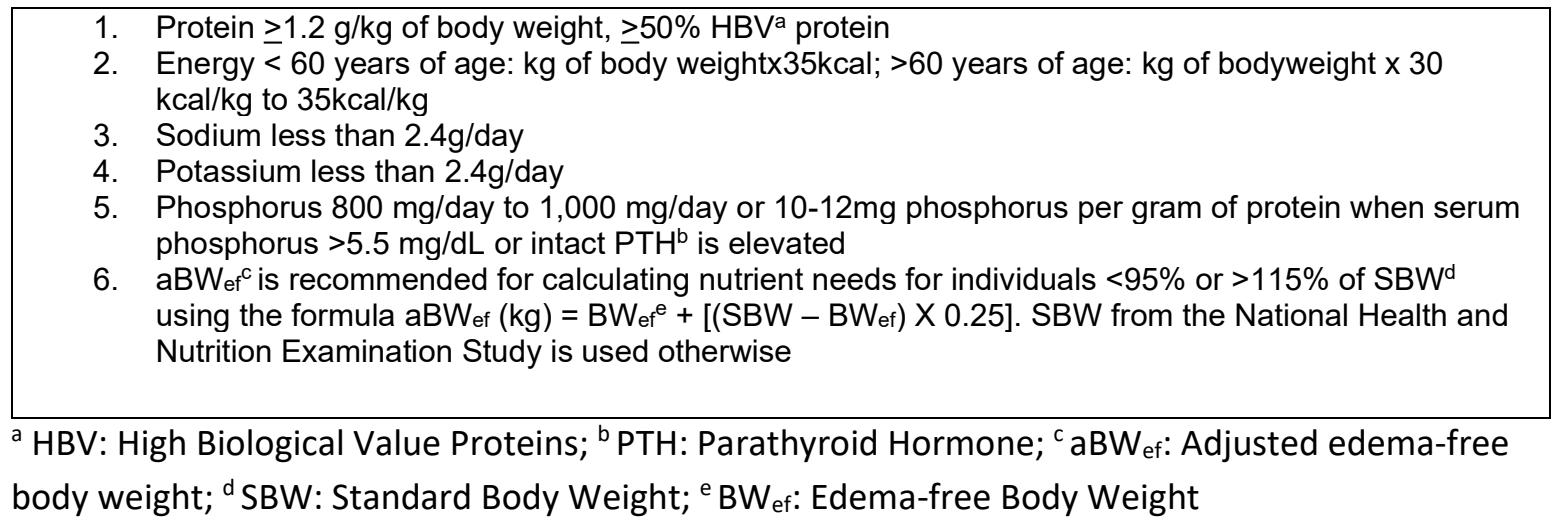

\section{BIOCHEMICAL PARAMETERS}

Serum phosphorus, potassium, and iron were retrieved from the patients' medical records. These biochemical parameters were measured as part of the routine tests performed in the hemodialysis unit. All tests were conducted post-dialysis session. Comparative standards for serum potassium were considered between $3.5 \mathrm{mEq} / \mathrm{L}$ to $5.5 \mathrm{mEq} / \mathrm{L}$; and between $3.5 \mathrm{mg} / \mathrm{dL}$ to $5.5 \mathrm{mg} / \mathrm{dL}$ for phosphorus $[15,29]$.

\section{STATISTICAL ANALYSIS}

Shapiro Wilk normality test was performed to ensure that data is normally distributed. Cohen's $d$ effect sizes and confidence intervals (95\%) were derived from means and pooled standard deviations. The effect size was considered small at 0.2 , medium at 0.5 , and large at 0.8 and above [30]. Effect sizes were calculated to understand the magnitude of the reported effects along with the probability by means of p-values. [31]. Paired t-tests were performed to compare the mean scores before and after the intervention. Two-tailed p-values are reported. Frequencies and percentages were used to describe categorical variables; whereas means and standard deviations were used for continuous variables. Statistical software IBM SPPS 
Statistics 21 Data Editor was used to perform all statistical analyses (IBM Corp. Released 2012. IBM SPSS Statistics for Windows, Version 21.0. Armonk, NY: IBM Corp.).

\section{RESULTS}

Out of 149 patients at the hemodialysis unit, 26 were eligible, and 23 downloaded the app and completed the study. Participants that were not eligible consisted mainly of persons who did not own a smartphone or who owned a smartphone with an IOS operating system. Two subjects did not download the app due to limitations in phone storage, and one subject was not interested in downloading the app. Figure 2 depicts the CONSORT flow diagram. The sample's mean age was 48.5 (13.7), and mean BMI was 31.9 (7.9); subjects had been on dialysis for a mean of 29.7 (37.3) months. More than half of the subjects were males (14), and more than half suffered from hypertension and/or diabetes. Demographic data are detailed in Table 1.

Figure 2. CONSORT study flow diagram

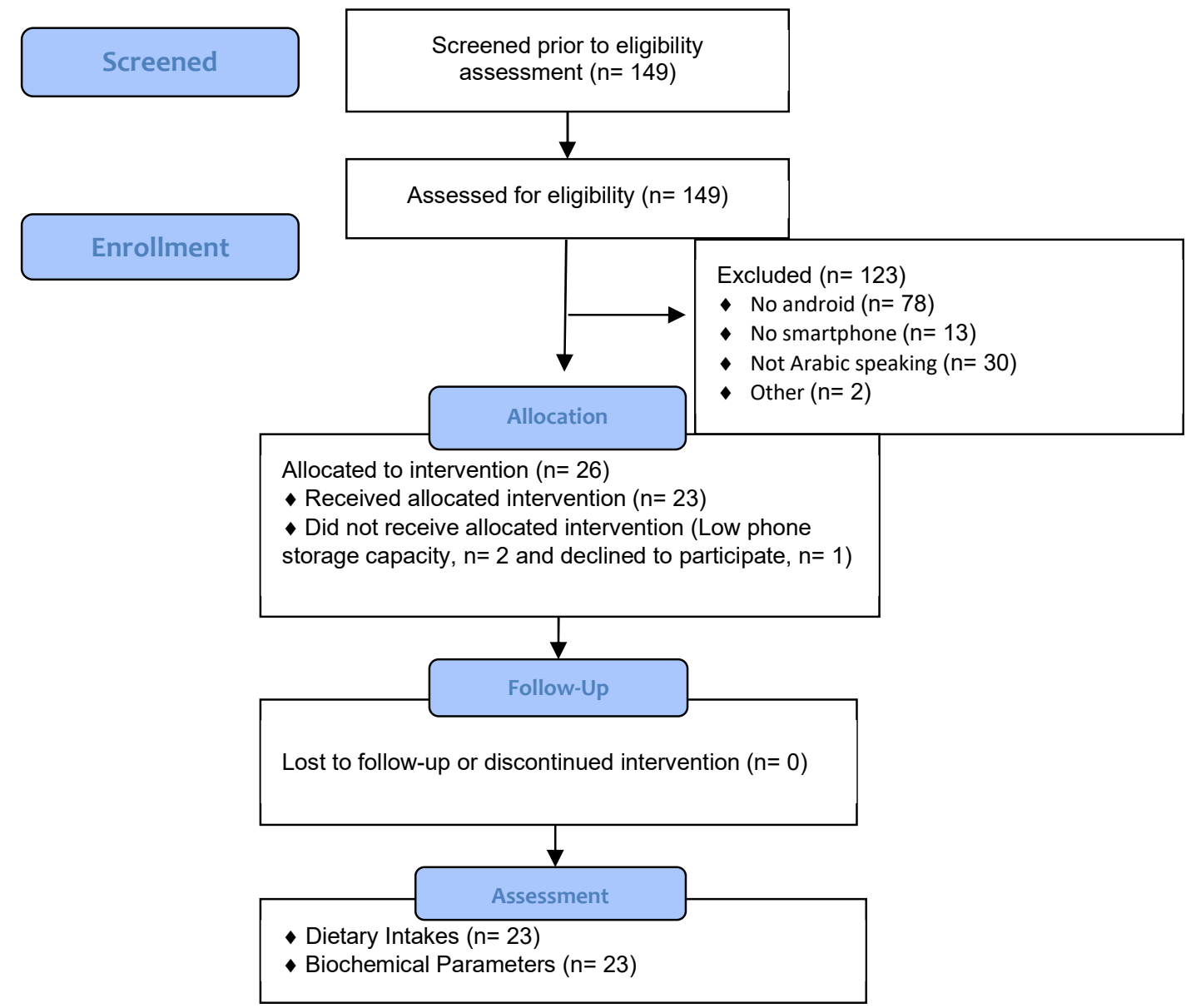


Table 1: Demographic and baseline characteristics of the sample $(N=23)$

\begin{tabular}{|l|l|}
\hline & Mean (SD) \\
\hline Age & $48.5(13.7)$ \\
\hline BMI $^{\mathbf{a}}$ & $31.9(7.9)$ \\
\hline Months on Dialysis & $29.7(37.3)$ \\
\hline Gender & $\mathbf{n}(\%)$ \\
\hline Male & \\
\hline Smokers & $14(61)$ \\
\hline Comorbidities & $6(26)$ \\
\hline HTN & \\
\hline Diabetes & $16(70)$ \\
\hline Dyslipidemia & $11(48)$ \\
\hline Cancer & $2(9)$ \\
\hline Liver Disease & $1(4)$ \\
\hline
\end{tabular}

${ }^{a}$ BMI: Body Mass Index; ${ }^{b} \mathrm{HTN}$ : Hypertension

\section{ANTHROPOMETRY AND DIETARY INTAKES}

There was no change in weight and BMI post-intervention; however, mean dietary intakes changed mainly for energy and macronutrients. Mean energy intakes increased from 24.4 $\mathrm{Kcal} / \mathrm{kg} / \mathrm{d}(8.0)$ to $29.1 \mathrm{Kcal} / \mathrm{kg} / \mathrm{d}(7.8)$ with a medium effect size $(d=0.6 ; 95 \% \mathrm{CI} 0.0-1.2)$. Mean protein intakes increased from $0.9 \mathrm{~g} / \mathrm{kg} / \mathrm{d}(0.3)$ to $1.3 \mathrm{~g} / \mathrm{kg} / \mathrm{d}(0.5)$ with a large effect size $(d=1.0 ; 95 \% \mathrm{CI} 0.4-1.6)$; intake of high biological value proteins $(\% \mathrm{HBV})$ also increased from $58.6 \%(10.1)$ to $70.1 \%(10.7)$ with a large effect size $(d=1.1 ; 95 \% \mathrm{CI} 0.5-1.7)$. Total fat intakes increased from baseline with medium effect size $(d=0.5 ; 95 \%$ CI $0.0-1.1)$. Dietary intakes of minerals did not change, apart from sodium which decreased from an intake of $2218.8 \mathrm{mg} / \mathrm{d}$ (631.6) to $1895.3 \mathrm{mg} / \mathrm{d}(581.0)$ with a medium effect size $(d=0.5 ; 95 \% \mathrm{CI} 0.1-1.1)$.

\section{BIOCHEMICAL PARAMETERS}

Serum phosphorus, potassium, and albumin did not change relevantly. Serum iron increased from $7.9 \mathrm{mg} / \mathrm{dl}(2.8)$ to $11.5 \mathrm{mg} / \mathrm{dl}$ (7.9) post-intervention with a medium effect size $(\mathrm{d}=0.6$; $95 \%$ CI $0.0,1.2$ ). Table 2 details the results of the anthropometry, dietary intakes, and blood parameters. 
Table 2. Dietary intakes and laboratory data at baseline and post-intervention

\begin{tabular}{|c|c|c|c|c|}
\hline & Baseline (TO) & $\begin{array}{l}\text { Post-Intervention } \\
\text { (T1) }\end{array}$ & Cohen's d $(95 \% \mathrm{Cl})$ & $P$-value \\
\hline & Mean (SD) & Mean (SD) & & \\
\hline Weight & $85.5(23.1)$ & $84.1(24.1)$ & $0.1(-0.5,0.6)$ & .37 \\
\hline $\mathrm{BMI}^{\mathrm{a}}$ & $31.9(7.9)$ & $31.3(8.5)$ & $0.1(-0.5,0.6)$ & .39 \\
\hline $\begin{array}{l}\text { Energy Intake } \\
\text { (Kcal/d) }\end{array}$ & $1918.3(570.4)$ & $2206.2(378.2)$ & $0.6(0.0,1.2)$ & $.003 *$ \\
\hline $\begin{array}{l}\text { Energy Intake } \\
\text { (Kcal/kg/d) }\end{array}$ & $24.4(8.0)$ & $29.1(7.8)$ & $0.6(0.0,1.2)$ & $.002 *$ \\
\hline $\begin{array}{l}\text { Dietary Protein } \\
\text { (g/d) }\end{array}$ & $71.1(26.4)$ & $103.8(37.8)$ & $1.0(0.4,1.6)$ & $<.001 *$ \\
\hline $\begin{array}{l}\text { Dietary Protein } \\
(\mathrm{g} / \mathrm{kg} / \mathrm{d})\end{array}$ & $0.9(0.3)$ & $1.3(0.5)$ & $1.1(0.4,1.7)$ & $<.001^{*}$ \\
\hline $\mathrm{HBV}^{\mathrm{b}}(\%)$ & $58.6(10.1)$ & $70.1(10.7)$ & $1.1(0.5,1.7)$ & $<.001^{*}$ \\
\hline $\begin{array}{l}\text { Total Dietary } \mathrm{CHO}^{\mathrm{c}} \\
\text { (g/d) }\end{array}$ & $224.7(88.0)$ & $215.2(40.7)$ & $0.1(-0.4,0.7)$ & .59 \\
\hline $\begin{array}{l}\text { Total Dietary Fat } \\
(\mathrm{g} / \mathrm{d})\end{array}$ & $87.3(30.1)$ & $103.0(26.8)$ & $0.5(0.0,1.1)$ & $.020 *$ \\
\hline $\begin{array}{l}\text { Dietary Potassium } \\
(\mathrm{mg} / \mathrm{d})\end{array}$ & $1831.2(728.4)$ & 2046.1 (555.1) & $0.3(0.2,0.9)$ & .19 \\
\hline $\begin{array}{l}\text { Dietary } \\
\text { Phosphorus (mg/d) }\end{array}$ & $1152.5(489.7)$ & $1343.1(398.0)$ & $0.4(0.2,1.0)$ & .09 \\
\hline $\begin{array}{l}\text { Dietary Sodium } \\
(\mathrm{mg} / \mathrm{d})\end{array}$ & $2218.8(631.6)$ & $1895.3(581.0)$ & $0.5(0.1,1.1)$ & $.03 *$ \\
\hline Serum K (mg/dl) & $4.7(0.7)$ & $4.7(0.7)$ & $0.0(-0.6,0.6)$ & .92 \\
\hline Serum $P$ (mg/dl) & $5.2(1.5)$ & $5.5(2.2)$ & $0.15(-0.4,0.7)$ & .60 \\
\hline Serum Fe (mg/dl) & $7.9(2.8)$ & $11.5(7.9)$ & $0.6(0.0,1.2)$ & $.03 *$ \\
\hline Serum Al (g/dl) & $3.0(0.4)$ & $3.2(0.8)$ & $0.3(-0.3,0.9)$ & .37 \\
\hline
\end{tabular}

${ }^{*} P<.05$; ${ }^{\mathrm{a} B M I}$ : Body Mass Index; ${ }^{\mathrm{b}} \mathrm{HBV}$ : High Biological Value Proteins; ${ }^{\mathrm{C}} \mathrm{CHO}$ : Carbohydrates

\section{ADHERENCE TO DIETARY GUIDELINES IN HEMODIALYSIS}

Adherence to dietary guidelines in hemodialysis improved for energy intakes, protein intakes, and high biological value proteins with medium to large sizes, respectively $(\mathrm{d}=0.4 ; 95 \% \mathrm{CI}$ 0.2-1.0 for energy, $\mathrm{d}=0.9$; CI $0.3,1.5$ for proteins, $\mathrm{d}=1.1 ; 95 \% \mathrm{CI} 0.5-1.7)$. Adherence to fat, potassium, and phosphorus intakes did not change. Whereas adherence to sodium further dropped to achieve intakes below $2400 \mathrm{mg} / \mathrm{d}$ with a medium effect size $(\mathrm{d}=0.5$; CI $0.1-1.1)$. The number of patients adhering to dietary guidelines increased for energy, proteins, and sodium, whereas a larger number of patients became non-adherent to fat and phosphorus intakes. There was no relevant change in the number of patients concerning adherence to potassium intakes. Table 3 describes the difference between the recommended intakes and dietary intakes before and after the intervention. Figure 3 details the results of percent compliance to dietary recommendations. 
Table 3. Adherence to dietary intakes reported as the difference between intake and recommendation at baseline and post-intervention

\begin{tabular}{|l|l|l|l|l|}
\hline & Baseline & Post-Intervention & Cohen's d (95\% Cl) & $P$-value \\
\hline & Mean (SD) & Mean (SD) & & \\
\hline Energy (Kcal/d) & $-903.1(705.4)$ & $-581.3(779.8)$ & $0.4(0.2,1.0)$ & $.004^{*}$ \\
\hline Dietary Protein (g/d) & $-27.3(26.6)$ & $+6.5(43.9)$ & $0.9(0.3,1.5)$ & $<.001^{*}$ \\
\hline HBV $^{\mathbf{a}}$ (\%) & $58.6(10.1)$ & $70.1(10.7)$ & $1.1(0.5,1.7)$ & $<.001^{*}$ \\
\hline Total Dietary Fat (\% Energy) & $41.0(7.6)$ & $41.9(7.3)$ & $0.1(-0.7,0.4)$ & .66 \\
\hline Dietary Potassium (mg/d) & $-568.1(728.4)$ & $-353.9(555.1)$ & $0.3(-0.9,0.2)$ & .19 \\
\hline Dietary Phosphorus (mg/d) & $+84.7(445.8)$ & $250.3(369.9)$ & $0.4(-0.9,0.2)$ & .14 \\
\hline Dietary Sodium (mg/d) & $-181.1(631.6)$ & $-504.6(581.0)$ & $0.5(0.1,1.1)$ & $.03^{*}$ \\
\hline
\end{tabular}

aHBV: High Biological Value Proteins

\section{Compliance to Dietary Guidelines in Hemodialysis}

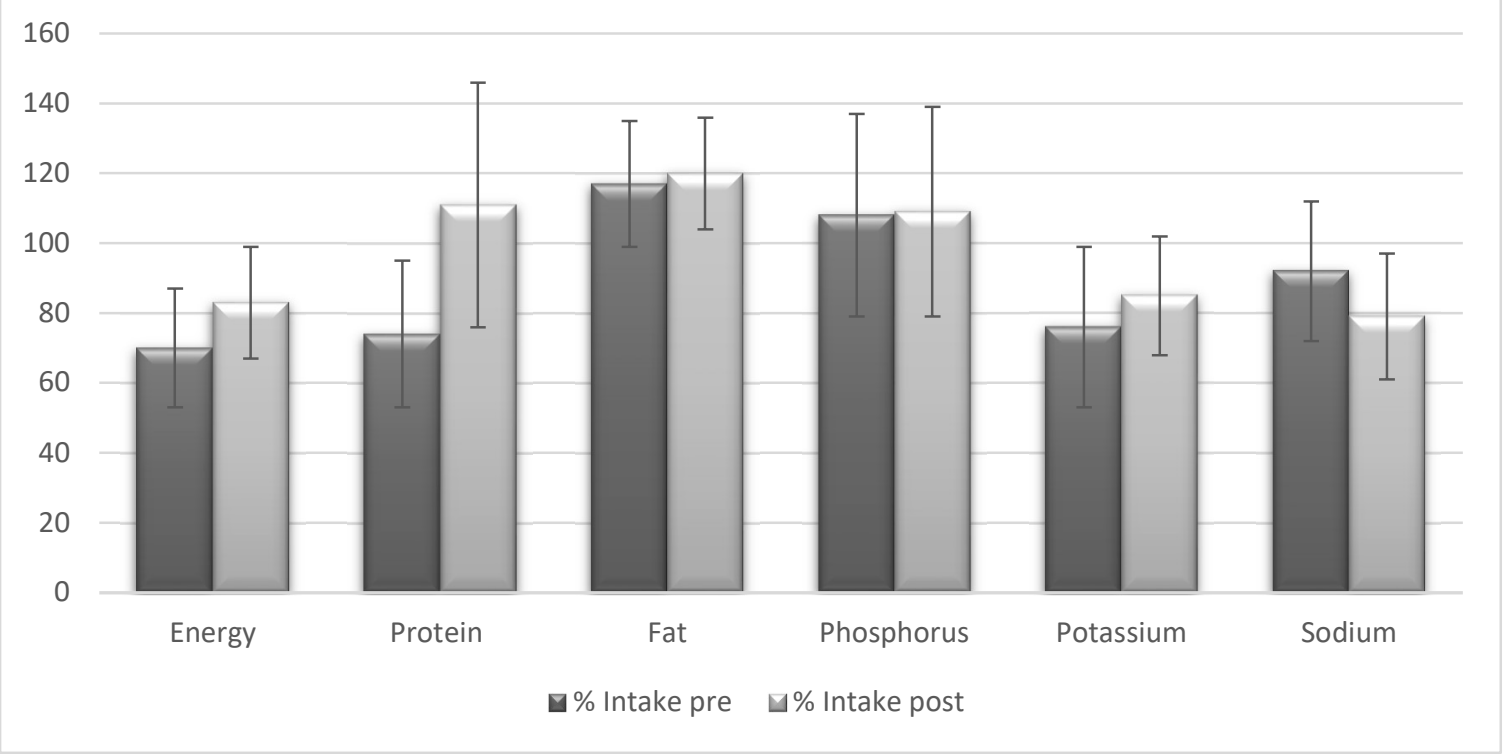

Figure 3. \% Adherence to Dietary intakes pre- and post-intervention (mean \pm SD)

\section{USER ACCEPTABILITY AND APP USABILITY}

A user acceptability tool was used during this pilot; however, all participants responded to all questions with a "strongly agree" based on a Likert scale. Accordingly, the results were not considered useful to understand better the acceptability and, therefore, have not been reported. Assessment of acceptability will be reassessed in the future trial. The validated mobile app rating scale end-user version of the Mobile App Rating Scale (uMARS) [32] will be used in the trial. Participants may not have well understood that the questionnaire aims to improve the app; thus, qualitative data will also be collected to ask specific questions of the KELA.AE usability and acceptability by referring to each feature in the app individually. Qualitative data will also address the culturally specific features of the app such as language and recipes. 
Field notes revealed that all 23 participants attended the weekly sessions with the dietitians. Usage data available from analytics was only able to provide the last date of access for each participant, and all 23 had accessed the app in the last week of the trial. No data on usage frequency was able to be retrieved due to a lack of in-app analytics.

\section{DISCUSSION}

\section{PRINCIPAL RESULTS}

\section{Potential Efficacy}

In this study, short-term app usage had a potential impact on energy and protein intakes among hemodialysis patients. In comparison to the target dietary guidelines for hemodialysis, subjects started with a baseline intake of energy and proteins below recommendations. As an outcome of the intervention, energy intakes reached an average of $29.1 \mathrm{Kcal} / \mathrm{kg} / \mathrm{d}(7.8)$ as compared to the recommended $30-35 \mathrm{Kcal} / \mathrm{kg} / \mathrm{d}$. Additionally, protein intakes were also considerably low as compared to guidelines (mean consumption at baseline of $0.9 \mathrm{~g} / \mathrm{kg} / \mathrm{d}$ ) and improved to become in line with the recommended $\geq 1.2 \mathrm{~g} / \mathrm{kg}$ of body weight, $\geq 50 \% \mathrm{HBV}$ protein $[15,29]$.

No changes were observed in intakes of minerals; nonetheless, baseline intakes of potassium, and sodium were already within the recommendations of less than $2400 \mathrm{mg} / \mathrm{d}$. Sodium intakes further dropped to an average of $1895.3 \mathrm{mg} / \mathrm{d}$ (581.0). Fat and phosphorus intakes, however, were already elevated at baselines and remained above recommendations after the intervention.

No changes were observed in the serum laboratory parameters for potassium and phosphorus; levels remained within recommendation at baseline and after the intervention. The duration of the intervention is not long enough to detect relevant changes in serum values; accordingly, the upcoming trial is expected to capture the effect of app usage on laboratory parameters better. Serum Fe increased with a medium effect size from $7.9 \mathrm{mg} / \mathrm{dl}$ (2.8) to $11.5 \mathrm{mg} / \mathrm{dl}$ postintervention. The latter may be explained by the increase in the dietary protein of high biological value, which mainly consists of animal protein sources.

\section{Study and app feasibility}

Research methodology, resources, and tools used in this pilot were assessed to refine and modify the planned trial and improve the app. The feasibility of the processes related to recruitment, retention, and refusal seemed adequate, there were no patients that withdrew from the study, and all eligible patients agreed to participate. However, the number of eligible subjects were about $20 \%$ out of all dialysis patients at the unit. It would mean that to achieve an appropriate sample size for the planned randomized trial, and many dialysis units will need to be recruited. Thus, the number of dietitians needed to be involved in the future study will need adequate resources to meet patients weekly, as it was the case in this study. Accordingly, 
an assessment of resource allocation should be performed to consider app improvement and development on IOS mobile operating system to increase eligible patients within the same dialysis unit.

Digital interventions may be used as tools to support a reciprocal relationship between patients and healthcare practitioners and enhance patient-centered care [33]. Accordingly, dietitians would be expected to manage and recommend the usage of KELA.AE app in real practice settings. Thus, the future trial will include dietitians following up with patients to simulate app integration in real practice.

In-app analytics are also essential for the future trial to assess the effect of app usage on outcomes. In-app analytics should track usage frequency as well as the usage of each feature separately. The results would help to better understand if educational or self-monitoring features of the app are preferred by the user.

The future trial should further analyze the fatty acid profile (saturated, monounsaturated, and polyunsaturated) on top of total fats to assess diet quality from an atherogenic point of view. Additionally, further assessment of the nutritional status of dialysis patients should be evaluated to identify malnourished patients that may need additional support from the research dietitians.

The KELA.AE app will be re-evaluated to include educational materials addressing diet quality pertinent to dietary fatty acid profiles to promote dietary intakes in line with the K/DOQI clinical practice guidelines for the management of dyslipidemias in patients with kidney disease [34]. This is an update to be considered in the next version of the app shall dietary fat intakes not be in line with guidelines.

This pilot study has demonstrated that it is feasible to integrate a dietary app into dietetic practice allowing it to be a tool in addition to regular meetings with dietitians. The study has also identified how to adjust the design, procedures, data collection tools, and outcome measurements for a future trial. Based on the information collected, we feel ready to proceed to a larger randomized controlled trial.

\section{COMPARISON WITH PRIOR WORK}

Energy and protein recommendations in hemodialysis are higher than the recommendations for healthy subjects. Whereas in the general population, intakes of $0.8 \mathrm{~g} / \mathrm{kg}$ are adequate to maintain nitrogen balance [35], such intakes are inadequate in hemodialysis [36]. Hemodialysis sessions cause losses in proteins and amino acids that need to be replenished by the diet [36]. Protein- 
energy wasting is dangerous and has been correlated with mortality, adverse clinical outcomes [37], and poor quality of life [18]. Inadequate dietary proteins and energy intakes are both criteria used in the diagnosis of protein-energy wasting in hemodialysis patients [38]. Similarly, to the findings in this pilot, many studies have identified inadequate energy and protein intakes among dialysis patients $[14,39,40]$. This pilot intervention seems to show potential in the improvement of energy and protein intakes among this population.

However, multiple dietary components need to be adjusted to improve the clinical outcomes of these patients, and the latter includes management of potassium, phosphorus, along with a diet quality that is cardio protective [15]. In the sample studied in this pilot, both serum potassium levels and potassium intakes were within targets before and after the intervention. This was also observed by others $[19,14]$, whereby similarly to our findings, hemodialysis patients tend to be adherent to potassium intakes and laboratory targets. However, serum phosphorus and phosphorus intakes were borderline high both before and after the intervention. This is also a common finding in this population; whereby the discrepancies between the phosphorus and protein recommendations [15] is challenging to achieve. Accordingly, it seems that further assessment of the factors that may influence phosphorus management, such as food sources of phosphorus and compliance to phosphate binders, should be explored. Additionally, it is expected that a longer intervention that includes education and follow up with dietitians may lead to better outcomes.

Total fat intakes were also found to be elevated by our pilot study, and their intakes remained elevated after the intervention. Others have previously explored the diet quality of hemodialysis patients as compared to the recommendations of the American Heart Association and KDOQI Clinical Practice Guidelines for Management of Dyslipidemias in Patients With Kidney Disease [34]; and found the current diet intakes to be proatherogenic in nature [14]. Given that protein intakes increased and that their sources are mainly from animal proteins (an increase in $\%$ high biological value proteins were also observed), it can be predicted that saturated fat intakes also increased as a result of these changes.

There are only few available apps that target patients with chronic kidney disease (CKD) specifically; however, most of them are available on app stores, and information on their efficacy and usability are rather scarce [41]. A recent content analysis of mobile apps for chronic kidney disease revealed that available apps fail to provide the continuity of patientcentered care that is needed to support CKD patients [42]. 


\section{LIMITATIONS}

Given the pilot nature of the study, some limitations may lead to a bias in the interpretation of the results and their generalizability. The duration of this study was short; therefore, only effects are focused on dietary intakes and laboratory parameters. Behavioral interventions need to be of longer duration and should be comprehensive to change dietary intakes [43]. The results of this pilot will instead be used to improve the app and modify the study procedures in the future trial.

Additionally, the results identified a possible improvement in nutritional status and a potentially proatherogenic diet quality among the study's sample. Thus, the future trial should assess the prevalence of malnourished patients along with the fatty acid profile of the diet in line with the KDOQI Clinical Practice Guidelines for Management of Dyslipidemias in Patients With Kidney Disease [34].

The intervention included face to face sessions with dietitians that may have influenced the outcomes. The reinforcement of the dietitians and their availability may have impacted the results similarly or more than the app itself. Accordingly, the future trial should include a control group whereby dietitians see patients with the same frequency but without app usage.

Given that app analytics were not available, we were unable to track how many times the users accessed the app. The only available data is the last access for each user, and all users had accessed the app during the last week.

\section{CONCLUSIONS}

This pilot study showed that KELA.AE app has the potential to improve dietary intakes. Processes related to the procedures, resources, tools, and app improvement for a future trial were assessed. A more extended intervention using a randomized controlled trial is required to estimate parameters concerning app efficacy accurately.

\section{ACKNOWLEDGMENTS}

We acknowledge the staff and students from Zayed University who volunteered by contributing to the app development at many steps. 


\section{REFERENCES}

1. WorldhealthOrganization WHO. mHealth: use of appropriate digital technologies for public health. March 2018. In.

2. Zaidan S, Roehrer E. Popular mobile phone apps for diet and weight loss: a content analysis. JMIR mHealth and uHealth. 2016;4(3):e80.

3. El Khoury CF, Karavetian M, Halfens RJ, Crutzen R, Khoja L, Schols JM. The Effects of Dietary Mobile Apps on Nutritional Outcomes in Adults with Chronic Diseases: A Systematic Review and Meta-Analysis. Journal of the Academy of Nutrition and Dietetics. 2019;119(4):626-651.

4. Helle C, Hillesund ER, Wills AK, Øverby NC. Evaluation of an eHealth intervention aiming to promote healthy food habits from infancy-the Norwegian randomized controlled trial Early Food for Future Health. International Journal of Behavioral Nutrition and Physical Activity. 2019;16(1):1.

5. Järvelä-Reijonen E, Karhunen L, Sairanen E, et al. The effects of acceptance and commitment therapy on eating behavior and diet delivered through face-to-face contact and a mobile app: a randomized controlled trial. international journal of behavioral nutrition and physical activity. 2018;15(1):22.

6. Gonzalez-Sanchez J, Recio-Rodriguez JI, Fernandez-delRio A, et al. Using a smartphone app in changing cardiovascular risk factors: A randomized controlled trial (EVIDENT II study). International journal of medical informatics. 2019;125:13-21.

7. Lemacks JL, Adams K, Lovetere A. Dietary Intake Reporting Accuracy of the Bridge2U Mobile Application Food Log Compared to Control Meal and Dietary Recall Methods. Nutrients. 2019;11(1):199.

8. Burke LE, Wang J, Sevick MA. Self-monitoring in weight loss: a systematic review of the literature. Journal of the American Dietetic Association. 2011;111(1):92-102.

9. Kerr D, Dhaliwal S, Pollard C, et al. BMI is associated with the willingness to record diet with a mobile food record among adults participating in dietary interventions. Nutrients. 2017;9(3):244.

10. Fakih El Khoury C, Karavetian M, Halfens RJ, Crutzen R, El Chaar D, Schols JM. Dietary Application for the Management of Patients with Hemodialysis: A Formative Development Study. Healthcare Informatics Research. 2019;25(4):262-273.

11. Turner-McGrievy GM, Dunn CG, Wilcox S, et al. Defining Adherence to Mobile Dietary Self-Monitoring and Assessing Tracking Over Time: Tracking at Least Two Eating Occasions per Day Is Best Marker of Adherence within Two Different Mobile 
Health Randomized Weight Loss Interventions. Journal of the Academy of Nutrition and Dietetics. 2019.

12. Kelly MP, Barker M. Why is changing health-related behaviour so difficult? Public health. 2016;136:109-116.

13. Hayashi A, Yamaguchi S, Waki K, et al. Testing the feasibility and usability of a novel smartphone-based self-management support system for dialysis patients: a pilot study. JMIR research protocols. 2017;6(4):e63.

14. Luis D, Zlatkis K, Comenge B, et al. Dietary quality and adherence to dietary recommendations in patients undergoing hemodialysis. Journal of Renal Nutrition. 2016;26(3):190-195.

15. Kopple JD. National kidney foundation K/DOQI clinical practice guidelines for nutrition in chronic renal failure. American journal of kidney diseases. 2001;37(1):S66-S70.

16. Tentori F, Blayney MJ, Albert JM, et al. Mortality risk for dialysis patients with different levels of serum calcium, phosphorus, and PTH: the Dialysis Outcomes and Practice Patterns Study (DOPPS). American Journal of Kidney Diseases. 2008;52(3):519-530.

17. Kovesdy CP, Regidor DL, Mehrotra R, et al. Serum and dialysate potassium concentrations and survival in hemodialysis patients. Clinical Journal of the American Society of Nephrology. 2007;2(5):999-1007.

18. Borges MCC, Vogt BP, Martin LC, Caramori JCT. Malnutrition Inflammation Score cutoff predicting mortality in maintenance hemodialysis patients. Clinical nutrition ESPEN. 2017; 17:63-67.

19. Kim H, Lim H, Choue R. A better diet quality is attributable to adequate energy intake in hemodialysis patients. Clinical nutrition research. 2015;4(1):46-55.

20. Rollo ME, Burrows T, Vincze LJ, Harvey J, Collins CE, Hutchesson MJ. Cost evaluation of providing evidence - based dietetic services for weight management in adults: In person versus eHealth delivery. Nutrition \& dietetics. 2018;75(1):35-43.

21. Ni Z, Jin H, Jiang G, et al. A Telemedicine-Based Registration System for the Management of Renal Anemia in Patients on Maintenance Hemodialysis: Multicenter Study. Journal of Medical Internet Research. 2019;21(5):e13168.

22. Viechtbauer W, Smits L, Kotz D, et al. A simple formula for the calculation of sample size in pilot studies. Journal of clinical epidemiology. 2015;68(11):1375-1379.

23. Prochaska JO, Velicer WF. The transtheoretical model of health behavior change. American journal of health promotion. 1997;12(1):38-48.

24. Fishbein M. A reasoned action approach to health promotion. Medical Decision Making. 2008;28(6):834-844. 
25. Mummah SA, Robinson TN, King AC, Gardner CD, Sutton S. IDEAS (Integrate, Design, Assess, and Share): a framework and toolkit of strategies for the development of more effective digital interventions to change health behavior. Journal of medical Internet research. 2016;18(12):e317.

26. Lancaster GA, Thabane L. Guidelines for reporting non-randomised pilot and feasibility studies. In: Springer; 2019.

27. Schulz KF, Altman DG, Moher D. CONSORT 2010 statement: Updated guidelines for reporting parallel group randomized trials. Annals of Internal Medicine. 2011;154(4):291-292.

28. Nutrition Ao, Dietetics, Van Horn L, Beto JA. Research: Successful Approaches in Nutrition and Dietetics. Academy of Nutrition and Dietetics; 2019.

29. Nutrition Care Manual. Academy of Nutrition and Dietetics; 2019. Accessed September 2019.

30. Cohen J. A power primer. Psychological bulletin. 1992;112(1):155.

31. Lakens D. Calculating and reporting effect sizes to facilitate cumulative science: a practical primer for t-tests and ANOVAs. Frontiers in psychology. 2013;4:863.

32. Stoyanov SR, Hides L, Kavanagh DJ, Wilson H. Development and validation of the user version of the Mobile Application Rating Scale (uMARS). JMIR mHealth and uHealth. 2016;4(2):e72.

33. Ammenwerth E. From eHealth to ePatient: the role of patient portals in fostering patient empowerment. European Journal of Biomedical Informatics. 2018;14(2).

34. Initiative KDOQ. K/DOQI clinical practice guidelines for management of dyslipidemias in patients with kidney disease. American journal of kidney diseases: the official journal of the National Kidney Foundation. 2003;41(4 Suppl 3):I.

35. Trumbo P, Schlicker S, Yates AA, Poos M. Dietary reference intakes for energy, carbohdrate, fiber, fat, fatty acids, cholesterol, protein and amino acids. Journal of the Academy of Nutrition and Dietetics. 2002;102(11):1621.

36. Sabatino A, Regolisti G, Karupaiah T, et al. Protein-energy wasting and nutritional supplementation in patients with end-stage renal disease on hemodialysis. Clinical nutrition. 2017;36(3):663-671.

37. Kovesdy CP. Malnutrition in dialysis patients - the need for intervention despite uncertain benefits. Paper presented at: Seminars in dialysis2016.

38. Fouque D, Kalantar-Zadeh K, Kopple J, et al. A proposed nomenclature and diagnostic criteria for protein-energy wasting in acute and chronic kidney disease. Kidney international. 2008;73(4):391-398. 
39. Carvalho KT, Silva MIB, Bregman R. Nutritional profile of patients with chronic renal failure. Journal of Renal Nutrition. 2004;14(2):97-100.

40. Kopple JD, Berg R, Houser H, Steinman TI, Teschan P. Nutritional status of patients with different levels of chronic renal insufficiency. Kidney International Supplement. 1989(27).

41. Lewis RA, Lunney M, Chong C, Tonelli M. Identifying Mobile Applications Aimed at Self-Management in People With Chronic Kidney Disease. Canadian Journal of Kidney Health and Disease. 2019;6:2054358119834283.

42. Lee Y-L, Cui Y-Y, Tu M-H, Chen Y-C, Chang P. Mobile health to maintain continuity of patient-centered care for chronic kidney disease: content analysis of apps. JMIR mHealth and uHealth. 2018;6(4):e10173.

43. Nooriani N, Mohammadi V, Feizi A, Shahnazi H, Askari G, Ramezanzade E. The effect of nutritional education based on health belief model on nutritional knowledge, Health Belief Model constructs, and dietary intake in hemodialysis patients. Iranian journal of nursing and midwifery research. 2019;24(5):372. 

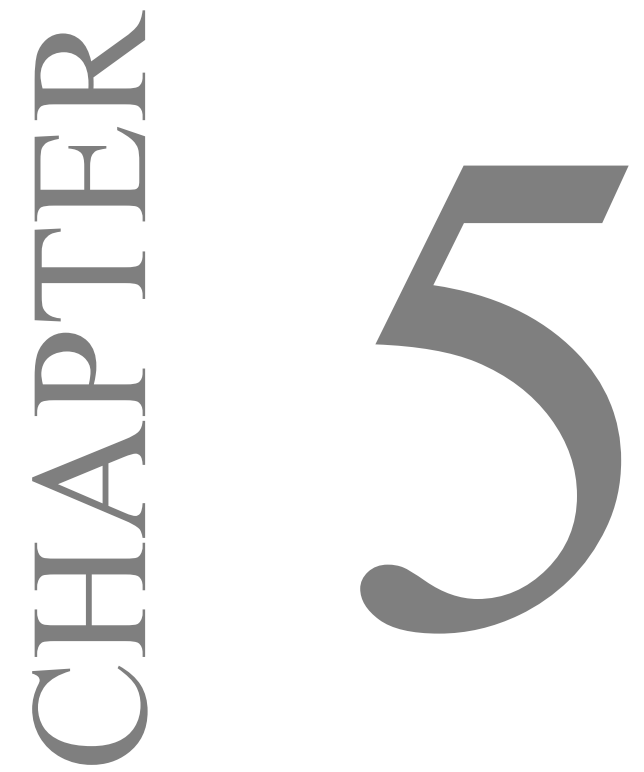

\section{ADEQUATE MANAGEMENT OF PHOSPHORUS IN HEMODIALYSIS USING A DIETARY SMARTPHONE APP: A PILOT STUDY}

Cosette Fakih El Khoury, Rik Crutzen, Jos M.G.A. Schols, Ruud J.G. Halfens and Mirey Karavetian

JMIR Preprints. 17/01/2020:17858 (submitted) 


\begin{abstract}
Background: The renal diet is complex and requires amendments in various nutrients. Elevated serum phosphorus is common among hemodialysis patients, and it is associated with many complications. Alternative approaches that support both dietitians and patients in overcoming these difficulties should be explored. Smartphone technology could be used to provide a source of accessible and reliable information.
\end{abstract}

Objective: The aim of this pilot is to assess the potential efficacy of an intervention using KELA.AE on the phosphorous management in hemodialysis patients. Results will be used to improve both the application and a planned, rigorous large-scale trial intended to assess app efficacy.

Methods: This is a pilot study performed at the hemodialysis unit of Al Qassimi Hospital (Emirate of Sharjah). All patients were assessed for eligibility, and based on inclusion criteria, they were considered for enrollment. Participants met with a dietitian once a week and used the mobile app. Outcomes were measured at baseline (T0) and two weeks post app usage (T1). This pilot is reported as per guidelines for non-randomized pilot and feasibility studies and in line with the CONSORT 2010 checklist for reporting pilot or feasibility trials.

Results: Twenty-three subjects successfully completed the pilot. Patients knowledge improved to $68.1 \%$ (13.3) after intervention with a large effect size $(\mathrm{d}=1.22,95 \% \mathrm{CI}$ 0.59-1.85). Dietary protein intakes increased from a mean of $0.9 \mathrm{~g} / \mathrm{Kg}(\mathrm{SD}=0.3)$ per day to a mean of $1.3 \mathrm{~g} / \mathrm{Kg}$ $(\mathrm{SD}=0.5)$ per day with a large effect size $(\mathrm{d}=1.07,95 \% \mathrm{CI} 0.45-1.69)$. Phosphorus to protein ratio dropped from a mean of $18.4 \mathrm{mg} / \mathrm{g}$ protein to $13.5 \mathrm{mg} / \mathrm{g}$ protein with a large effect size $(d=0.83,95 \%$ CI $0.22-1.43)$. There were no improvements in phosphorous intakes, selfreported non-adherence, and serum phosphorus.

Conclusions: The findings of this pilot reveal potential efficacy for the use of a smartphone app as a supportive nutrition education tool for the phosphorus management in hemodialysis. This pilot study showed that KELA.AE app has the potential to improve knowledge and dietary choices. Processes related to the procedure, resources, tools, and app improvement for a future trial were assessed. A rigorous randomized controlled trial should be performed to evaluate the efficacy, assessing app usage of a long-term intervention.

\title{
KEYWORDS
}

Renal diet, mhealth, dietary app 


\section{INTRODUCTION}

Dietary management plays an essential role in slowing down disease progression and improving the quality of life of people with chronic kidney disease [1,2]. However, the renal diet is complex and requires targeted attention for the intakes of various nutrients [3]; and patients often express frustration and confusion in this regard [4].

Elevations in blood phosphorus are specifically common among this patient group, and they are associated with many comorbidities such as cardiovascular disease, metabolic bone mineral disease and mortality [5]. Even a mild hyperphosphatemia greater than $5.0 \mathrm{mg} / \mathrm{dl}$ is independently associated with an increase in mortality among hemodialysis patients [6]. Therefore, the management of hyperphosphatemia is essential during hemodialysis; however, this seems to be particularly challenging. Patients are recommended to consume $1.2 \mathrm{~g} / \mathrm{kg}$ body weight of protein, in order to achieve protein needs during hemodialysis [3]. Moreover, phosphorus is typically found in protein-rich foods, thus restricting dietary phosphorus to the recommended $800-1000 \mathrm{mg} / \mathrm{d}$, while maintaining adequate protein intakes is the cornerstone of this diet [3]. Such recommendations are not easily compatible [7] and lead to conflicting and ambiguous information that requires simplifications and clarification from the dietitian's side [8].

Adherence to the renal diet is, therefore, an essential component for the management of hemodialysis patients [9]. Patient knowledge can play a role in compliance to the diet [10]. Therefore, nutrition education and counseling might have a positive effect on blood phosphorous [11]. However, eating habits are complicated and not merely influenced by knowledge, but also by a patient's readiness to change and their health beliefs [12]. The association between knowledge and adherence is not always clear and an increase in knowledge does not necessarily imply improved adherence [13]. Nutrition education can, nevertheless, improve nutrition knowledge, which, in turn, can support increased dietary adherence $[14,15]$. However, this knowledge is especially valuable when patients are willing to change their dietary behaviors [13].

From the patient's perspective, receiving easy to understand individualized nutrition education at the early stages is desired [4]. Educational materials should be theory-based and adapted to the patient [2] in order to be effective. A high-intensity education has been listed among the facilitators for improvements of serum phosphorus [16]. Time limitation, however, during the encounter with dietitians and physicians often acts as a barrier to effective nutrition education [17].

A recent study qualitatively explored the experience of renal dietitians. Dietitians expressed frustration, limited resources, and emotional and professional challenges in providing dietary 
education to chronic kidney disease patients [8]. Hemodialysis patients show similar frustration and a need for continuous access to reliable nutrition information. Therefore, alternative approaches should be explored that may support both dietitians and patients in overcoming these difficulties. Smartphone technology can provide persons with chronic diseases with accessible and reliable information [18]. Additionally, in-app educational materials allow patients access to nutrition education in different modalities [19].

Accordingly, dietary apps can be effective at improving nutritional outcomes in chronic diseases [20].

The IDEAS (Integrate, Design, Assess and Share) framework for the development of effective digital interventions defines the process of assessing the efficacy of a developed product to start with a pilot study aiming at estimating potential efficacy. Information gathered from the pilot study would then be used to improve the product itself as well as the study design of a rigorous randomized controlled trial [21].

This pilot study aims at exploring the potential of phosphorus management in hemodialysis patients after the usage of a smartphone app. The description of the person-centered and theory-based app development is detailed in a separate publication [19]. This pilot study is in preparation for evaluating efficacy with a rigorous randomized control trial.

\section{METHODS}

\section{STUDY DESIGN}

This is a prospective pilot study that was conducted using a theory-based educational dietary app (KELA.AE). This pilot followed the guidelines for reporting non-randomized pilot and feasibility studies [22]. This study is also reported in line with the CONSORT 2010 checklist of information to include when reporting a pilot or feasibility trial excluding items pertinent to randomization $[22,23]$.

\section{PARTICIPANTS, ELIGIBILITY, AND RECRUITMENT}

A list of all hemodialysis patients was obtained from the hemodialysis unit, and all subjects were approached during their scheduled dialysis session to identify those that meet the inclusion criteria. Subjects identified were contacted and signed consent was received if they agreed to participate. A total of 26 participants were recruited from Al Qassimi Hospital dialysis center in the Emirate of Sharjah, United Arab Emirates. Patients on hemodialysis for at least three months, free of life-threatening conditions, able to read, write, listen and communicate in Arabic, owning an android smartphone and not having been hospitalized in the past six months were eligible to participate in the study. Recruitment and data collection 
were started during February 2019 and completed during April 2019. Post-hoc calculations of sample size in pilot studies, assuming detection of unanticipated problems with a probability of at least $15 \%$ probability ( $\pi=0.15$ ) and a $95 \%$ confidence level, resulted in a required sample of 19 subjects [24].

\section{ETHICAL APPROVALS}

The study received Institutional Review Board (IRB) approval from Zayed University, Dubai (Ethical Approval number ZU17_066_F) and was funded by a research grant provided by Zayed University, Dubai (Research Grant number R18060). All participants signed informed consent.

\section{PROCEDURE}

Participants were provided with a username to initiate the sign-in procedure. Upon registration, participants were provided with a brief orientation to the app features. Subsequently, free access to KELA.AE was provided for two weeks. During this period, participants were provided with nutrition support by the research team once a week. The team consisted of two licensed dietitians that provided participants with further nutrition education and clarifications upon request. All questions about the app usage and content were also answered. Baseline and post-intervention outcomes were assessed before app registration (T0) and after the completion of 2 weeks of app usage (T1). The trial was stopped when all participants completed two weeks of app usage. Participants could keep using the app if they wished so; however, all data collection was completed two weeks after app usage.

\section{KELA.AE APP}

The app consists of an Arabic, theory-based, and culture-specific android application (KELA.AE). A formative study of the app developed has been detailed elsewhere [19]. The app was designed to provide dietary education to hemodialysis patients along with traditional renal friendly recipes. The transtheoretical model [25] along with constructs from the Reasoned Action Approach [26], were incorporated in the development of the educational materials. Different educational modalities are included: notifications, podcasts and videos. Selfmonitoring features to track food intake and blood tests are also available.

\section{SELF-REPORTED NON-ADHERENCE}

The Dialysis Diet and Fluid non-adherence questionnaire (DDFQ) was used to assess selfreported dietary non-adherence [27]. The questionnaire includes two simple questions on the frequency and degree of non-adherence. It requires the patient to report non-adherence as the number of non-adherent days in the last 14 days. The degree of non-adherence is reported on a 
Likert scale ( 0 to 4 , where 0 means no and 4 means very severe non-adherence). The questions were translated to Arabic but given their straightforward simplicity further validation was deemed unnecessary. Construct validity of the original tool was reported using Kendall Tau correlation for frequency and degrees of non-adherence $(\tau=0.495 ; p<.001)$ [27].

\section{KNOWLEDGE}

The Knowledge questionnaire (KnQ) consists of 18 questions assessing knowledge about the renal diet. A total of 18 points can be achieved if all answers are correct; scores were then converted into a percentage. This tool was used in Arabic before [28] based on an adaptation of the original questionnaire [29] and now including Arabic foods. A score of $60 \%$ on overall knowledge (all 18 questions) was considered as sufficient knowledge [28]. Questions were clustered by topic and a sub-analysis was performed to understand knowledge in specific areas pertinent to phosphorous management (phosphorus content in food, consequences of hyperphosphatemia and use of phosphate binders).

\section{DIETARY INTAKES AND BIOCHEMICAL PARAMETERS}

Dietary intake was assessed using face-to-face 24-hr recalls [30]. Participants were asked if the day before was deemed representative of the previous week, and if not, adjustments were made based on participants' recall for better representation of the past week's intakes.

Phosphorus needs were considered as $1000 \mathrm{mg} / \mathrm{d}$ for participants with serum phosphorus below $5.5 \mathrm{mg} / \mathrm{d}$ and $12 \mathrm{mg}$ per gram of protein intake for participants with serum phosphorus below $5.5 \mathrm{mg} / \mathrm{dl}[31]$.

Blood parameters were retrieved from patient medical records as part of the routine protocols of the hemodialysis unit (measurements are taken post hemodialysis session). Target values for serum phosphorus in hemodialysis was considered between 3.5 and $5.5 \mathrm{mg} / \mathrm{dl}$ based on the National Kidney Foundation Kidney Disease Outcomes Quality Initiative (NFK KDOQI) recommendations [3].

\section{ANTHROPOMETRIC MEASUREMENTS}

Bodyweight and height were retrieved from patient medical records, as measured post-dialysis routinely. BMI was calculated accordingly using measured body weight and height. Comparative standards for body weight were used as suggested by the Nutrition Care Manual [31] based on the NFK KDOQI guidelines [3]. Accordingly, Standard body weight (SBW) from the National Health and Nutrition Examination Study was used for calculation of nutrient needs. Adjusted edema-free body weight (aBWef) was used for the calculation of nutrient needs for participants with $<95 \%$ or $<115 \%$ of standard body weight (SBW) [31]. 


\section{STATISTICAL ANALYSIS}

The software IBM SPPS Statistics 21 Data Editor was used to perform all statistical analyses (IBM Corp. Released 2012. IBM SPSS Statistics for Windows, Version 21.0. Armonk, NY: IBM Corp.). Categorical variables were described using frequencies and percentages, while means and standard deviations were used to represent continuous variables. Shapiro Wilk normality test was performed to ensure that data is normally distributed. Paired t-tests were used to compare the mean scores before and after the intervention. Two-tailed p-values are reported. Effect sizes were calculated as Cohen's d (with 95\% Confidence Intervals [19]) using mean difference and pooled standard deviations. The effect size was considered small at 0.2 , medium at 0.5 and large at 0.8 and above [32].

\section{RESULTS}

Twenty-three participants completed the app pilot testing. Two subjects were excluded due to issues related to low smartphone storage capacity, and one subject was not interested in downloading the app. The mean age of the participants was $48.5(\mathrm{SD}=13.7)$ years, and mean BMI was 31.9 ( $\mathrm{SD}=7.9)$, mean time on dialysis was over one year with 29.7 (37.3) months of dialysis. More men were enrolled in the study (61\%) and most participants suffered from hypertension $(70 \%)$ or diabetes $(48 \%)$. Demographics measured at baseline are shown in Table 1. Figure 1 depicts the CONSORT flow diagram.

Table 1: Demographics and baseline characteristics of the study population ( $N=23)$

\begin{tabular}{l|l}
\hline Variable & M (SD) \\
\hline Age & $48.5(13.7)$ \\
\hline BMIa & $31.9(7.9)$ \\
\hline Months on Dialysis & $29.7(37.3)$ \\
\hline Variable & $\mathrm{n}(\%)$ \\
\hline Gender & \\
\hline Male & $14(61)$ \\
\hline Female & $9(39)$ \\
\hline Smokers & $6(26)$ \\
\hline Comorbidities & \\
\hline HTN & $16(70)$ \\
\hline Diabetes & $11(48)$ \\
\hline Dyslipidemia & $2(9)$ \\
\hline Cancer & $1(4)$ \\
\hline Liver Disease & $1(4)$ \\
\hline BMI: Body Mass Index; bTN: Hypertension
\end{tabular}




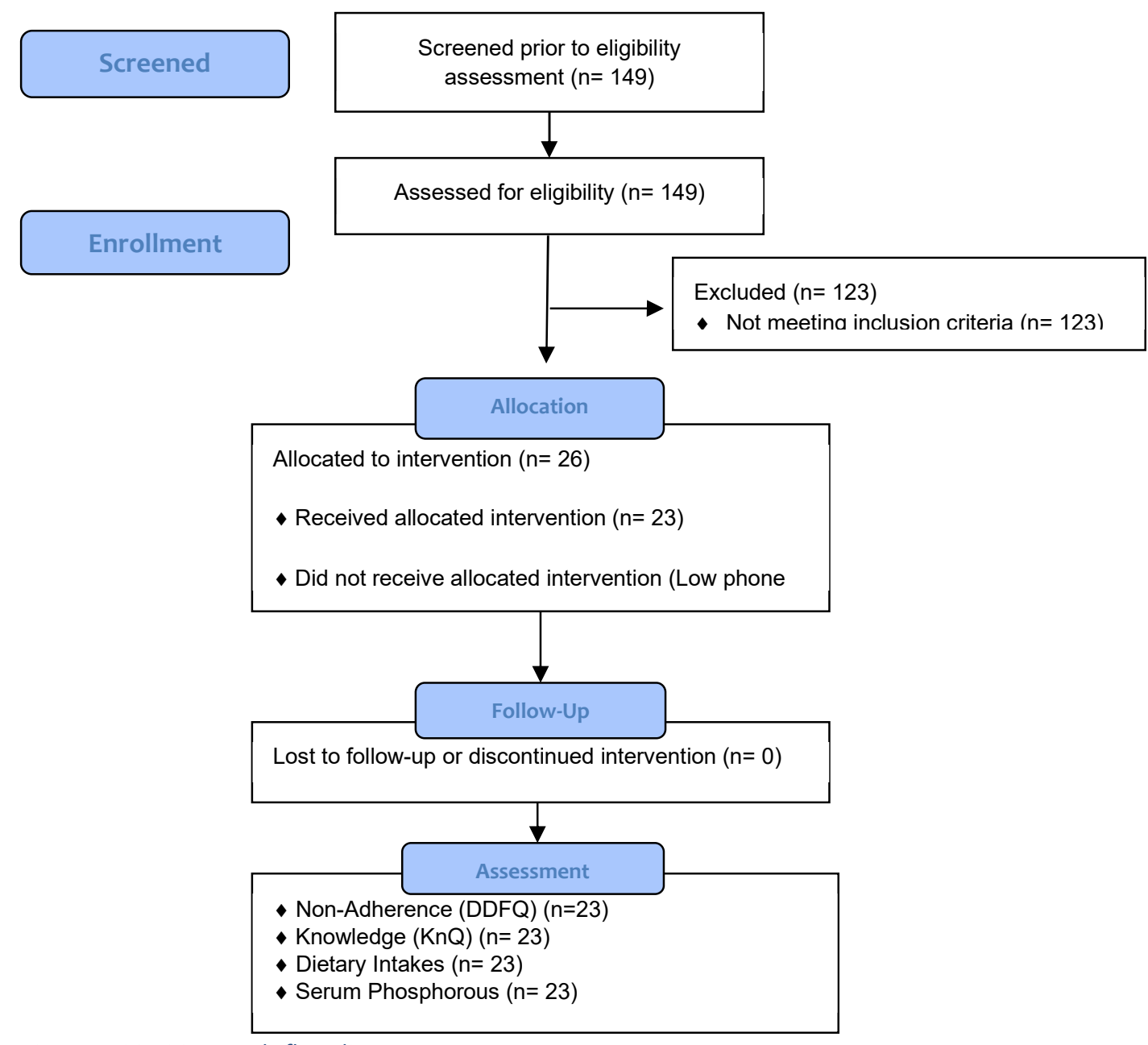

Figure 1: CONSORT study flow diagram

\section{KNOWLEDGE AND NON-ADHERENCE}

Overall, the mean self-reported non-adherence days dropped from $3.2(\mathrm{SD}=4.5)$ over the past 14 days to $2.0(\mathrm{SD}=3.0)$ after exposure to in-app education with small effect size $(\mathrm{d}=0.33$, 95CI -0.25-0.91). The severity of non-adherence was mostly mild and moderate pre- and post-intervention.

The mean perceived days of non-adherence to the phosphorus content of diet increased from $1.1(\mathrm{SD}=3.2)$ days to $1.9(\mathrm{SD}=3.0)$ days with small effect size $(\mathrm{d}=0.25,95 \% \mathrm{CI} 0.33-0.83)$. The severity of non-adherence changed from 19 participants reporting no deviation from the diet to only ten reporting no deviation.

Knowledge was below the $60 \%$ cut-off point of adequate knowledge at baseline and improved to reach a mean of $68.1 \%$ (13.3) after intervention with a large effect size $(d=1.22$, CI 0.59 , 1.85). Specific knowledge pertinent to consequences of hyperphosphatemia and phosphate binders also improved (large effect sizes $\mathrm{d}=1.15$, CI $0.53,1.77$ and $\mathrm{d}=1.00$, CI $0.39,1.61$ respectively). Knowledge about the phosphorus content of food improved with a medium effect 
size $(\mathrm{d}=0.54$, CI $-0.05,1.12)$. Results pertinent to adherence and knowledge are detailed in Table 2.

Table 2: Baseline and post-intervention self-reported dietary nonadherence and knowledge $(n=23)$

\begin{tabular}{|c|c|c|c|c|}
\hline & Baseline & $\begin{array}{l}\text { Post- } \\
\text { intervention }\end{array}$ & $\begin{array}{l}\text { Cohen's d } \\
(95 \% \mathrm{Cl})\end{array}$ & p-value \\
\hline $\begin{array}{l}\text { Dietary non-adherence } \\
\left(\mathrm{DDFQ}^{\mathrm{a}}\right)\end{array}$ & \multicolumn{2}{|c|}{ Mean (SD) } & & \\
\hline $\begin{array}{l}\text { Days of non-adherence } \\
\text { (overall diet) }\end{array}$ & $3.2(4.5)$ & $2.0(3.0)$ & $\begin{array}{l}0.33(-0.25- \\
0.91)\end{array}$ & .32 \\
\hline $\begin{array}{l}\text { Degree of non-adherence } \\
\text { (overall) }\end{array}$ & \multicolumn{2}{|c|}{ Frequency $n(\%)$} & & \\
\hline $\begin{array}{l}\text { 0-No deviation } \\
\text { 1-Mild } \\
\text { 2-Moderate } \\
\text { 3-Severe } \\
\text { 4-Very Severe }\end{array}$ & $\begin{array}{l}11(48) \\
5(22) \\
4(17) \\
2(9) \\
1(4)\end{array}$ & $\begin{array}{l}11(48) \\
4(18) \\
7(30) \\
1(4) \\
0(0)\end{array}$ & & \\
\hline $\begin{array}{l}\text { Dietary non-adherence } \\
\left(\text { DDFQ }^{\text {a) }} \text { Phosphorus }\right.\end{array}$ & \multicolumn{2}{|c|}{ Mean (SD) } & & \\
\hline $\begin{array}{l}\text { Days of non-adherence } \\
\text { (Phosphorus) }\end{array}$ & $1.1(3.2)$ & $1.9(3.0)$ & $\begin{array}{l}0.25(0.33- \\
0.83)\end{array}$ & .45 \\
\hline Degree of non-adherence & \multicolumn{2}{|c|}{ Frequency $n(\%)$} & & \\
\hline $\begin{array}{l}\text { 0-No deviation } \\
\text { 1-Mild } \\
\text { 2-Moderate } \\
\text { 3-Severe } \\
\text { 4-Very Severe }\end{array}$ & $\begin{array}{l}19(83) \\
1(4) \\
2(9) \\
0(0) \\
1(4)\end{array}$ & $\begin{array}{l}10(44) \\
5(22) \\
7(30) \\
1(4) \\
0(0)\end{array}$ & & \\
\hline Knowledge $\left(\mathrm{KnQ}^{\mathrm{b}}\right)$ & \multicolumn{2}{|c|}{ Mean (SD) } & & \\
\hline $\begin{array}{l}\text { \% Overall Knowledge } \\
\text { questionnaire }\end{array}$ & $51.4(13.9)$ & $68.1(13.3)$ & $\begin{array}{l}1.22(0.59- \\
1.85)\end{array}$ & $<.001^{*}$ \\
\hline $\begin{array}{l}\text { Knowledge of } \\
\text { Phosphorous content of } \\
\text { food (over } 7 \text { questions) }\end{array}$ & $47.8(21.4)$ & $57.1(12.2)$ & $\begin{array}{l}0.54(-0.05- \\
1.12)\end{array}$ & .06 \\
\hline $\begin{array}{l}\text { Knowledge of } \\
\text { Consequences of high } \\
\text { blood Phosphorous (over } 4 \\
\text { questions) }\end{array}$ & $43.5(18.8)$ & $66.3(20.8)$ & $\begin{array}{l}1.15(0.53- \\
1.77)\end{array}$ & $<.001^{*}$ \\
\hline $\begin{array}{l}\text { Knowledge of Phosphate } \\
\text { binders (over } 4 \text { questions) }\end{array}$ & $48.9(29.6)$ & $76.1(24.4)$ & $\begin{array}{l}1.00(0.39- \\
1.61)\end{array}$ & $<.001^{*}$ \\
\hline
\end{tabular}

${ }^{*} \mathrm{p}<.05 ;{ }^{\mathrm{a}}$ DDFQ: Dialysis Diet and Fluid non-adherence questionnaire; ${ }^{\mathrm{b}} \mathrm{KnQ}$ : Knowledge Questionnaire

\section{SERUM PHOSPHORUS AND DIETARY INTAKES}

Dietary protein intakes increased from a mean intake of $0.9 \mathrm{~g} / \mathrm{Kg}(\mathrm{SD}=0.3)$ per day to a mean intake of $1.3 \mathrm{~g} / \mathrm{Kg}(\mathrm{SD}=0.5)$ per day with a large effect size $(\mathrm{d}=1.07,95 \% \mathrm{CI} 0.45-1.69)$.

Phosphorus intakes as compared to phosphorus needs did not change; baseline and postintervention phosphorus intakes were below $115 \%$ of patients' needs. However, the phosphorus to protein ratio dropped from a mean of $18.4 \mathrm{mg} / \mathrm{g}$ protein to $13.5 \mathrm{mg} / \mathrm{g}$ protein with a large effect size $(d=0.83,95 \%$ CI $0.22-1.43)$. 
No changes were identified in serum phosphorus; however, the number of participants with serum phosphorus above $6 \mathrm{mg} / \mathrm{dl}$ increased to become ten subjects as compared to six at baseline. This may be in line with the increase in protein intakes and a slight increase in total phosphorus intakes. Table 3 illustrates data on serum phosphorus and dietary intakes.

Table 3: Baseline and post-intervention dietary intakes and serum phosphorus

\begin{tabular}{|c|c|c|c|c|}
\hline & Baseline & $\begin{array}{l}\text { Post- } \\
\text { intervention }\end{array}$ & $\begin{array}{l}\text { Cohen's d } \\
(95 \% \mathrm{Cl})\end{array}$ & p-value \\
\hline Phosphorus intakes & \multicolumn{2}{|c|}{ Mean (SD) } & & \\
\hline Dietary Phosphorus mg/d & $1152.5(489.8)$ & $1343.1(83.0)$ & $\begin{array}{l}0.42(-0.15- \\
1.01)\end{array}$ & .88 \\
\hline $\begin{array}{l}\text { \% compliance to } \\
\text { Phosphorus needs }\end{array}$ & $108.3(44.5)$ & $109.4(45.5)$ & $\begin{array}{l}0.02(-0.55- \\
0.60)\end{array}$ & .72 \\
\hline Dietary Protein $\mathrm{g} / \mathrm{d}$ & $71.1(26.4)$ & $103.8(37.8)$ & $\begin{array}{l}1.00(0.38- \\
1.61)\end{array}$ & $<.001^{*}$ \\
\hline Dietary Protein $\mathrm{g} / \mathrm{Kg} / \mathrm{d}$ & $0.9(0.3)$ & $1.3(0.5)$ & $\begin{array}{l}1.07(0.45- \\
1.69)\end{array}$ & $<.001^{*}$ \\
\hline $\begin{array}{l}\text { Phosphorus to Protein } \\
\text { ratio } \mathrm{mg} / \mathrm{g}\end{array}$ & $18.4(7.9)$ & $13.5(2.9)$ & $\begin{array}{l}0.83(0.22- \\
1.43)\end{array}$ & $.01^{*}$ \\
\hline Serum Phosphorus & \multicolumn{2}{|l|}{ Mean (SD) } & & \\
\hline Serum Phosphorus mg/dl & $5.3(1.5)$ & $5.5(2.0)$ & $\begin{array}{l}0.15(-0.43- \\
0.73)\end{array}$ & .60 \\
\hline $\begin{array}{l}\text { non-adherence to Serum } \\
\text { Phosphorus }\end{array}$ & \multicolumn{2}{|l|}{ Frequency $\mathrm{n}(\%)$} & & \\
\hline $\begin{array}{l}<5 \mathrm{mg} / \mathrm{dl} \\
5-6 \mathrm{mg} / \mathrm{dl} \\
>6 \mathrm{mg} / \mathrm{dl}\end{array}$ & $\begin{array}{l}11(48) \\
6(26) \\
6(26)\end{array}$ & $\begin{array}{l}11(48) \\
2(9) \\
10(43)\end{array}$ & & \\
\hline
\end{tabular}




\section{DISCUSSION}

The main findings of this pilot study show that in-app nutrition education, as a supportive tool to dietitians, can improve patient knowledge. Thus, this smartphone app might have potential as a useful source of nutrition education for dialysis patients. The use of smartphones as supportive tools to deliver education may help overcome the time limitation barrier that is reported during face to face encounters with dietitians and physicians [17]. Accordingly, their availability may be beneficial to both patients and healthcare practitioners as supportive tools for regular care.

However, adherence to the renal diet was not changed after the usage of the KELA.AE smartphone app. Serum phosphorus and phosphorus intakes did not improve either. Accordingly, the results may mean that knowledge does not necessarily increase adherence. Knowledge scores were not satisfactory at baseline (below the $60 \%$ cut-off used) and increased significantly to reach a mean score above $60 \%$. It seems that the association between knowledge and dietary non-adherence in dialysis patients is not always clear [33]. Surprisingly, in the results of this pilot, non-adherence to the phosphorus content of the diet increased after the intervention. The increase in knowledge may explain this. Patients might have become more aware of the phosphorus content of food and therefore, their selfperception of non-adherence changed accordingly. Thus, continuous education both by dietitians and in-app education material, may be needed to improve adherence and possibly serum phosphorus in the long run.

An increase in dietary protein was nevertheless observed as an outcome of the intervention. Protein intakes in hemodialysis are essential for the prevention of malnutrition. There is also an association between reduced protein intake and increased mortality [34]. The participants in this pilot study started with low protein intakes as compared to the needs of $\geq 1.2 \mathrm{~g} / \mathrm{Kg}$ per day recommended by NFK KDOQI guidelines [3] and achieved an average intake in line with recommendations post-intervention. The contradiction between the protein and phosphorous recommendations of the dialysis diet may lead to protein restriction as a result of decreased phosphorous intakes. Baseline and post-intervention phosphorus intakes were below $115 \%$ of patients' phosphorus recommendations. Therefore, the increase in protein intakes did not negatively impact phosphorus intakes. Additionally, the phosphorus to protein ratio post-intervention was closer to the recommended ratio of $10-12 \mathrm{mg} / \mathrm{g}$ protein [3], meaning that patients increased their protein intakes while choosing foods that were lower in phosphorus. These dietary changes may be a result of increased patient knowledge. However, the duration of the intervention may have been too short to detect changes in serum phosphorus, which is a limitation of the pilot. This will be better explored in the future trial. 
Adherence to phosphate binders is also an essential component in the phosphorous management of dialysis patients. In this pilot, knowledge pertinent to phosphate binders improved post-intervention, however, adherence to binders was not measured directly. Additionally, types of binders and their prescriptions were not investigated and benchmarked with recommendations.

Another limitation of the pilot study is the lack of app usage data, which was not retrievable due to the lack of in-app analytics. Therefore, the future trial should also be able to track inapp usage to assess if an increase in app usage influences improvement in outcomes.

This pilot aimed to assess and refine the methodology and procedures of a future trial aiming at detecting the efficacy of app usage in the adequate management of phosphorous in hemodialysis. Based on the findings, the planned trial should be designed to compare regular dietary interventions with dietary interventions supported by the app. During this study, participants often consulted with the research team throughout the regular weekly visits; thus, it would be important to explore further if the app enhances the phosphorous management or if it is instead the frequent dietary follow up that leads to improvements. In conclusion, the future trial should evaluate the usage of phosphate binders, app usage data and it should be designed in a way to detect if the app provides any advantages in the clinical outcomes of phosphorous management as compared to intensive dietary follow up by a dietitian.

\section{Conclusions}

The findings of this pilot study indicate that there is potential in the use of a smartphone app as a supportive nutrition education tool. A rigorous randomized controlled trial should be performed to evaluate the efficacy, in which app usage and long-term impact should be assessed.

\section{ACKNOWLEDGMENTS}

We acknowledge the staff and students from Zayed University who volunteered by contributing to the app development at many steps. 


\section{REFERENCES}

1. Palmer SC, Maggo JK, Campbell KL, et al. Dietary interventions for adults with chronic kidney disease. Cochrane Database of Systematic Reviews. 2017(4).

2. Beto JA, Ramirez WE, Bansal VK. Medical nutrition therapy in adults with chronic kidney disease: integrating evidence and consensus into practice for the generalist registered dietitian nutritionist. Journal of the Academy of Nutrition and Dietetics. 2014;114(7):1077-1087.

3. Kopple JD. National kidney foundation K/DOQI clinical practice guidelines for nutrition in chronic renal failure. American journal of kidney diseases. 2001;37(1):S66-S70.

4. Hollingdale R, Sutton D, Hart K. FACILITATING DIETARY CHANGE IN RENAL DISEASE: INVESTIGATING PATIENTS'PERSPECTIVES. Journal of Renal Care. 2008;34(3):136-142.

5. Abe M, Okada K, Soma M. Mineral metabolic abnormalities and mortality in dialysis patients. Nutrients. 2013;5(3):1002-1023.

6. Rodriguez-Benot A, Martin-Malo A, Alvarez-Lara MA, Rodriguez M, Aljama P. Mild hyperphosphatemia and mortality in hemodialysis patients. American journal of kidney diseases. 2005;46(1):68-77.

7. Shaman AM, Kowalski SR. Hyperphosphatemia management in patients with chronic kidney disease. Saudi Pharmaceutical Journal. 2016;24(4):494-505.

8. Lambert K, Mansfield K, Mullan J. Qualitative exploration of the experiences of renal dietitians and how they help patients with end stage kidney disease to understand the renal diet. Nutrition \& Dietetics. 2019;76(2):126-134.

9. Clark-Cutaia MN, Ren D, Hoffman LA, Burke LE, Sevick MA. Adherence to hemodialysis dietary sodium recommendations: influence of patient characteristics, selfefficacy, and perceived barriers. Journal of Renal Nutrition. 2014;24(2):92-99.

10. van Doorn-van Atten M, de Groot L, de Vries J, Haveman-Nies A. Determinants of Behaviour Change in a Multi-Component Telemonitoring Intervention for CommunityDwelling Older Adults. Nutrients. 2018;10(8):1062.

11. Caldeira D, Amaral T, David C, Sampaio C. Educational strategies to reduce serum phosphorus in hyperphosphatemic patients with chronic kidney disease: systematic review with meta-analysis. Journal of renal nutrition. 2011;21(4):285-294.

12. Elliott JO, Ortman C, Almaani S, Lee YH, Jordan K. Understanding the associations between modifying factors, individual health beliefs, and hemodialysis patients' adherence to a low-phosphorus diet. Journal of Renal Nutrition. 2015;25(2):111-120. 
13. Durose CL, Holdsworth M, Watson V, Przygrodzka F. Knowledge of dietary restrictions and the medical consequences of noncompliance by patients on hemodialysis are not predictive of dietary compliance. Journal of the American Dietetic Association. 2004;104(1):35-41.

14. Philippou E, Middleton N, Pistos C, Andreou E, Petrou M. The impact of nutrition education on nutrition knowledge and adherence to the Mediterranean Diet in adolescent competitive swimmers. Journal of science and medicine in sport. 2017;20(4):328-332.

15. Bonaccio M, Di Castelnuovo A, Costanzo S, et al. Nutrition knowledge is associated with higher adherence to Mediterranean diet and lower prevalence of obesity. Results from the Moli-sani study. Appetite. 2013;68:139-146.

16. Karavetian M, de Vries N, Rizk R, Elzein H. Dietary educational interventions for management of hyperphosphatemia in hemodialysis patients: a systematic review and meta-analysis. Nutrition reviews. 2014;72(7):471-482.

17. Anderson CA, Nguyen HA. Nutrition education in the care of patients with chronic kidney disease and end-stage renal disease. Paper presented at: Seminars in dialysis2018.

18. Gabrielli S, Dianti M, Maimone R, et al. Design of a mobile app for nutrition education (TreC-LifeStyle) and formative evaluation with families of overweight children. JMIR mHealth and uHealth. 2017;5(4):e48.

19. Fakih El Khoury C, Karavetian M, Halfens RJ, Crutzen R, El Chaar D, Schols JM. Dietary Application for the Management of Patients with Hemodialysis: A Formative Development Study. Healthcare Informatics Research. 2019;25(4):262-273.

20. El Khoury CF, Karavetian M, Halfens RJ, Crutzen R, Khoja L, Schols JM. The Effects of Dietary Mobile Apps on Nutritional Outcomes in Adults with Chronic Diseases: A Systematic Review and Meta-Analysis. Journal of the Academy of Nutrition and Dietetics. 2019;119(4):626-651.

21. Mummah SA, Robinson TN, King AC, Gardner CD, Sutton S. IDEAS (Integrate, Design, Assess, and Share): a framework and toolkit of strategies for the development of more effective digital interventions to change health behavior. Journal of medical Internet research. 2016;18(12):e317.

22. Lancaster GA, Thabane L. Guidelines for reporting non-randomised pilot and feasibility studies. In: Springer; 2019.

23. Schulz KF, Altman DG, Moher D. CONSORT 2010 statement: Updated guidelines for reporting parallel group randomized trials. Annals of Internal Medicine. 2011;154(4):291-292.

24. Viechtbauer W, Smits L, Kotz D, et al. A simple formula for the calculation of sample size in pilot studies. Journal of clinical epidemiology. 2015;68(11):1375-1379. 
25. Prochaska JO, Velicer WF. The transtheoretical model of health behavior change. American journal of health promotion. 1997;12(1):38-48.

26. Fishbein M. A reasoned action approach to health promotion. Medical Decision Making. 2008;28(6):834-844.

27. Vlaminck H, Maes B, Jacobs A, Reyntjens S, Evers G. The dialysis diet and fluid nonadherence questionnaire: validity testing of a self-report instrument for clinical practice INFORMATION POINT: Kendall's Tau. Journal of clinical nursing. 2001;10(5):707715.

28. Karavetian M, Abboud S, Elzein H, Haydar S, de Vries N. Nutritional education for management of osteodystrophy (NEMO) trial: Design and patient characteristics, Lebanon. Nutrition research and practice. 2014;8(1):103-111.

29. Ford JC, Pope JF, Hunt AE, Gerald B. The effect of diet education on the laboratory values and knowledge of hemodialysis patients with hyperphosphatemia. Journal of Renal Nutrition. 2004;14(1):36-44.

30. Nutrition Ao, Dietetics, Van Horn L, Beto JA. Research: Successful Approaches in Nutrition and Dietetics. Academy of Nutrition and Dietetics; 2019.

31. Nutrition Care Manual. Academy of Nutrition and Dietetics; 2019. Accessed September 2019.

32. Cohen J. A power primer. Psychological bulletin. 1992;112(1):155.

33. Miyata KN, Shen JI, Nishio Y, et al. Patient knowledge and adherence to maintenance hemodialysis: an International comparison study. Clinical and experimental nephrology. 2018;22(4):947-956.

34. Fouque D, Horne R, Cozzolino M, Kalantar-Zadeh K. Balancing nutrition and serum phosphorus in maintenance dialysis. American Journal of Kidney Diseases. 2014;64(1):143-150. 

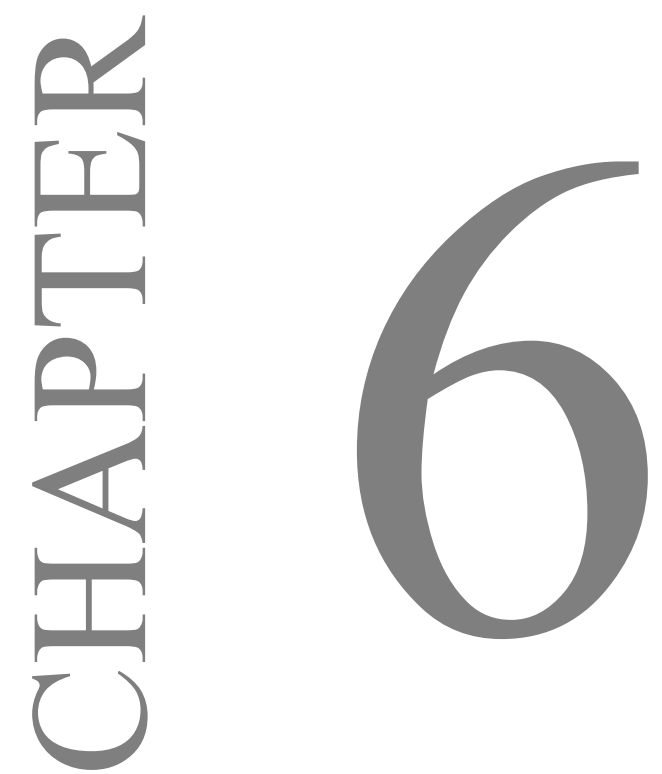

GENERAL DISCUSSION 
mHealth solutions can be supportive tools for patients, dietitians, and other healthcare practitioners; they can also influence the way patients have access to knowledge, healthcare services, and self-management tools $[1,2]$. App content, usability, and feasibility contribute to app effectiveness, and development strategies that are person-centered as well as grounded in theories and evidence are recommended $[3,4]$. The aim of this dissertation is to investigate the feasibility of using dietary mHealth interventions for hemodialysis patients. The following paragraphs describe the principal findings of this thesis and discuss methodological considerations along with implications for practice and future research.

\section{PRINCIPAL FINDINGS}

In Chapter 2, we performed a systematic review of the available literature along with a random-effect meta-analysis of interventions using dietary applications. Most of the identified studies targeted obesity and diabetes. Our findings confirmed that dietary mobile apps might have positive effects on nutritional outcomes. This is especially applicable to self-monitoring apps used in the short-term, and particularly in the case of weight management. Dietary apps that include elements based on a behavioral theory incorporated into a counseling intervention were found to be effective and thus may provide additional benefits. Our findings are compatible with the results of others who specifically investigated behavior theories used in mHealth and reported that theory-based interventions are crucial components that should be included during development. Salwen-Deremer et al. discussed how multiple traditional and contemporary theories could be applied to mHealth solutions that aim to address behavior changes [5].

While our results identified educational dietary apps as promising, not enough research is available in this regard. Most studies mainly report on health education interventions using technologies such as web-based dialogues, mobile texts, and video messages [6-8]. However, conclusive evidence on the effectiveness of in-app education in dietetics is still limited. Educational interventions using mHealth can address the characteristics and interests of users by overcoming barriers such as outreach, access to healthcare, time, and distance [9]. Thus, more research should be addressed at exploring the effectiveness of such interventions in dietetics.

The review and meta-analysis that we performed were not able to identify published studies on dietary smartphone apps in chronic kidney disease specifically. Previous reviews had identified renal apps available on Personal Digital Assistants (PDAs) rather than smartphones [10]. A more recent systematic review identified a total of nine articles, of which four did not report effectiveness and five reported effects on dietary behaviors; however, four out of five also used Personal Digital Assistants (PDAs) rather than smartphone apps. [11]. Therefore, available data 
on this subject is still scarce, and further research is needed to investigate the effectiveness of renal dietary smartphone apps.

In Chapter 3, we describe the formative development of an Arabic dietary renal app. Kidney Education for Lifestyle Application (KELA.AE app) is a self-monitoring and educational app for Arabic speaking hemodialysis patients. KELA.AE app was developed based on the Integrate, Design, Assess, and Share (IDEAS) Framework [4]. The development process used core processes to aid decision making and followed an in-person approach with the integration of evidence-based information and theories of behavior change.

Qualitative, semi-structured interviews with hemodialysis patients and healthcare practitioners (dietitians and nephrologists) were performed. The patients interviewed during the development reported using the Internet as a source for nutrition information, which is in line with previous findings [12]. Surprisingly, the results of the interviews with patients stressed a need for education rather than self-monitoring tools. Based on the findings of our systematic review detailed in Chapter 2, most evidence available is for self-monitoring dietary apps in chronic diseases rather than educational apps [2]. According to the findings of another systematic review, renal apps available mainly contain food diaries and dietary recommendations, whereas $\mathrm{CKD}$-friendly recipes and in-app education are not as common [11]. Therefore, available apps do not seem to cater to the needs of hemodialysis patients.

Qualitative data with healthcare practitioners, on the other hand, considered the KELA.AE app a useful tool for patients; however, they expressed concern regarding app misuse and replacement of healthcare practitioners. Others also describe the concern of healthcare practitioners; a study from Lebanon has reported a reluctance among physicians in the use of apps as communication tools [13]. The willingness of healthcare practitioners (physicians) to engage in a partnership with the patient seems to influence the success of digital interventions [14]. A qualitative study explored the perspectives of physicians and nurses, wherein participants described a shift in the patient-healthcare practitioner communication towards a more collaborative dialogue that can be enabled by digital tools [15].

The KELA.AE app was developed to contain in-app education consisting of podcasts, animated videos, and notifications. All educational materials were developed based on previously validated print Arabic dietary educational materials [16]. The materials amended to accommodate the needs of the participants' culture (e.g., Emirate dialect, local traditional recipes) and the educational modalities included in the app (e.g., podcasts, videos, and notifications). The validated materials were developed based on the Transtheoretical Model (TTM) for behavior change [17]. 
Different models of behavior change have been used in dietary mHealth interventions, among the most reported traditional models are the Social Cognitive Theory (SCT), the Reasoned Action Approach (RAA), and the Transtheoretical Model (TTM) [5]. Behavior change models often overlap, and constructs of a given theory adds upon the explanation of other theories. For instance, a study examining the characteristics associated with regular exercise found that the construct of self-efficacy in the Social Cognitive Theory (SCT) may be a predictor of the stages of the Transtheoretical Model (TTM) which also includes self-efficacy as a construct[18]. Accordingly, interactions across theories seem to apply, and multiple theories may be more relevant to a given behavior as compared to individual theories [5]. The TTM has often been critiqued in the literature with concern that the progression from stage to stage does not necessarily mean behavior change [19]. The strength of the TTM is that it views behavior change as a dynamic process [20]. However, the model proposes that individuals linearly progress through stages, whereas the process may be iterative, and individuals may cross back and forth through the different stages [21]. The assumption that individuals plan their actions within a definite timeframe (which is proposed by TTM) and that stages have clear boundaries dividing them have also been criticized [21]. The staging algorithm proposed by the model lacks validity; accordingly, some researchers have opted to adjust them; however, despite all the criticism, stage-based interventions have shown promising results [22]. Therefore, we have preserved the use of the validated stage-based materials; however, only three different stages of change (pre-action, action, and maintenance) were adopted rather than the five stages proposed by the model [16]. KELA.AE app re-assesses the stage of change automatically every month by asking the user to assess readiness for change. Accordingly, we have accommodated for an iterative movement between stages rather than a linear one. To adjust for the shortcomings of the model, and based on literature proposing interactions between models, we have included constructs of a different theory of change. Brainstorming sessions were performed as part of core processes and the literature was reviewed [20]. Consequently, we incorporated elements addressing self-efficacy, norms, and attitudes. The narration of the podcasts, video scripts and notifications was re-created to incorporate these additional constructs identified within the RAA [23].

After the app was developed, we conducted a pilot study to assess the feasibility and potential of KELA.AE app usage along with a face-to-face intervention. We present data regarding dietary intakes and diet quality (Chapter 4) and phosphorus management in hemodialysis (Chapter 5). The results from this pilot study should be used to improve the app itself as well as the study design of a future planned randomized controlled trial in the future. 
Our findings show that mHealth interventions using face-to-face encounters and apps may be feasible in the short-term and that possibly app usage could have a potential impact on some nutrient intakes among hemodialysis patients. Additionally, our pilot identified that in-app nutrition education could be a supportive tool to dietitians in terms of patient education and the improvement of patient knowledge. Patient knowledge can play a role in enhancing dietary adherence [24]. Our study, nevertheless, found no improvement in dietary adherence, and surprisingly non-adherence to the phosphorus content of the diet increased after the intervention. This may be explained by the self-perception of adherence influenced by patient knowledge. Our main conclusions pertain to the feasibility of the intervention, and they open room for future research on the efficacy of these interventions in the context of hemodialysis.

Non-compliance with dietary restrictions and particularly to phosphorous restrictions are common. The hemodialysis diet is somewhat restrictive and complicated, and patients often lack adequate knowledge to manage it [25]. Maintaining adequate intake of minerals (potassium, phosphorus, sodium and calcium) is essential in the prevention of dialysis-related complications such as heart failure, metabolic bone disorders, and mortality [26, 27]. Contradictory to restrictive mineral recommendations, protein intakes in hemodialysis should be higher than in the general population to avoid protein-energy malnutrition. The latter also contributes to complications among dialysis patients and is a predictor of mortality [28]. Coordinating between all these components requires a high level of knowledge and multiple, continuous counseling interventions. Dietary interventions for chronic kidney disease typically comprise in-person counseling sessions, which need to be tailored to the patient's needs [29]. Patients, nevertheless, have a desire for interventions that require less in-person contact with providers [30]. Similarly, continuous access to information and health care providers were also identified as patient needs [31].

\section{METHODOLOGICAL CONSIDERATIONS}

Several methodologies were used across this dissertation. Specific concerns, strengths, and limitations are discussed in each of the studies. The below paragraphs discuss the overall methodological approaches made along with strengths and limitations.

We have opted to use a mixed-methods approach to understand the current literature and available evidence, and the perspective of the multiple stakeholders involved in the usage of mHealth interventions. The study's strength lies in the involvement of patients and healthcare practitioners in app development. We considered the viewpoints of physicians and dietitians given the limitations in resources and time and given that they were mostly involved in the nutritional management of the patients at the hemodialysis unit piloted. However, we did not 
capture the point of view of nurses and family members who are often also involved in the care of hemodialysis patients.

Challenges were faced pertaining to the available literature in chronic kidney disease, and accordingly, some conclusions were drawn based on the evidence available for the management of obesity and diabetes. Additionally, our meta-analysis was mainly based on the findings from interventions using self-monitoring tools, which are not in line with the needs expressed by the patients. As discussed in a previous paragraph, information on mHealth in CKD and the effectiveness of in-app education in dietetics (which were identified as a need by patients) are scarce. Additionally, culturally specific published data is not available on Arabic apps; neither are tools for app development and evaluation tailored to users in the Arab world. Accordingly, we have assumed generalizability based on data derived mainly from western countries.

Based on the challenges faced, we have opted to combine the available knowledge from other chronic diseases identified through the meta-analysis, along with the insights provided by more recent reviews[11], theories, frameworks, and qualitative data gathered from patients and stakeholder to develop the KELA.AE app. This process, to our best knowledge, transformed the challenges into an opportunity to illustrate a comprehensive, iterative approach that accounts for multiple components.

The main limitation during app development was the incorporation of an English nutrient database into the self-monitoring feature of the app. Using nutrient data sources developed in different countries may lead to significant errors in the assessment of nutrient composition [32]. Nevertheless, this is an issue of concern across the Arab world as practitioners use different nutrient databases due to the lack of a reliable, comprehensive Arabic database. Available composition tables are mainly published as textbooks that are country specific [33], or outdated [34].

\section{IMPLICATIONS FOR PRACTICE}

Professional organizations in dietetics have acknowledged mobile apps as tools that can offer dietetic practitioners ways to communicate, engage, and monitor patients/clients [35]. The British Dietetics Association, for instance, considers mobile apps tools that can support dietitians in their practice [36]. The Academy of Nutrition and Dietetics in the United States of America considers nutrition informatics an area of practice that is rapidly evolving, and recommends that dietetic practitioners continually update their skills to remain at the vanguard of the practice [37]. 
Despite the promise of mobile apps, there remain controversies that should be examined from an ethical and social standpoint, in addition to their efficacy. The Academy council on Future practice findings of the Academy of Nutrition and Dietetics considered the rapid growth of technology to be unsettling for dietetics practice and recommended that dietitians be involved in the development of these technologies [37]. Others have highlighted the lack of synchrony between developers and researchers in delivering apps that are based on formative research yet not up to the consumers' expectations [38]. The involvement of dietetic practitioners in the development of dietary apps is recommended and may expand the reach of their practice.

We have faced similar controversies during the development of the KELA.AE app, whereby most of the available knowledge was not in synchrony with the needs expressed by patients. We have opted to involve local dietitians and physicians, on top of patients to avoid falling into these mishaps. As proposed by the IDEAS framework, dissemination is an essential component of app development; it starts with the dissemination of research related to app development and effectiveness, but it also pertains to the dissemination of the app or mHealth intervention itself $[4,39]$. Dissemination of research would imply publications, particularly in open access journals, to share results. App dissemination, on the other hand, may require collaboration with the industry and possibly commercialization of the app itself [39]. Another means of dissemination could occur through channels such as governmental bodies or medical/dietary associations, which would also allow for open access apps available to patients for free. When apps are disseminated by professional organizations and governmental bodies, their adoption by patients and healthcare practitioners may also increase, meaning that practitioners may start prescribing app usage or consider it part of the routine care of the patients. A multi-country study explored app usage among dietitians in Australia, New Zealand, and the United Kingdom and reported that nutrition apps are used by $62 \%$ of dietitians in their practice either as information sources or as patient self-monitoring tools [40]. The study also identified barriers to app usage to be a lack of access to smartphone devices at work, lack of Wi-Fi access, and lack of knowledge about which apps can be suggested to patients. Facilitators identified included training, education, and advocacy about dietary apps [40].

Additionally, a recent study qualitatively explored the experience of renal dietitians. Dietitians expressed frustration, limited resources, and emotional and professional challenges in providing dietary education to chronic kidney disease patients [41]. Such findings imply that healthcare practitioners may benefit from mHealth interventions as tools to support communication, monitoring, and education of their patients, and they may use apps as part of their practice. The role of the healthcare practitioner may favor app adoption by consumers as well. Consumers have reported preferring app usage as a complementation to in-person 
encounters with healthcare practitioners rather than as a replacement [42]. Accordingly, app dissemination through reliable channels such as professional associations and governmental bodies, followed by adoption by healthcare practitioners, may contribute to the success of mHealth interventions for patients.

Accordingly, digital interventions may be used as tools to support a reciprocal relationship between patients and healthcare practitioners and enhance patient-centered care [14].

Based on the challenges faced during the incorporation of food and nutrient databases into the KELA.AE app, online databases in the Arabic language that are updated continuously and include traditional foods are needed in the region. Given the rise in technology, countries must engage in the development of databases to facilitate research and development of nutritional sciences and diet-related digital interventions in the region. Such databases should be available online; they should be comprehensive, updated continuously, culturally specific, and of open access. The development of these databases requires national and regional involvement of governmental institutions in collaboration with international organizations.

\section{ETHICAL AND REGULATORY ASPECTS}

Many countries have already opted for mHealth initiatives; nevertheless, only a few of these initiatives have been evaluated [43]. Despite the potential of mHealth in increasing healthcare coverage globally, privacy and security concerns have been reported. Cookies, data mining and device theft are all possible concerns [44]. The European Union and the United States of America have set laws pertinent to security and privacy (EU Data Protection Directive 95/46/EC; Health Insurance Portability and Accountability Act (HIPAA) and Federal Trade Commission (FTC) Act to name some) [45]. In Arab countries, there is still a lack of eHealth national policies that are coordinated between countries. In scientific literature, the focus is rather on telemedicine and telecare instead of mobile health. mHealth initiatives in the United Arab Emirates are available; however, their effectiveness has not been evaluated rigorously [46]. A collaboration between academia/researchers and governmental institutions should support the development of national policies that focus on needs assessment, formative evidence-based development, and, most importantly, dissemination and adoption of mHealth interventions. Such interventions should be then monitored, evaluated, and continuously improved in order to maximize their effectiveness.

Apprehensions have also risen on the recognition of effective apps and the classification of apps that may have diagnostic and therapeutic potentials [47]. Only a few apps are subject to regulation, and they mainly consist of high-risk health apps that have medical purposes. Many available apps, however, target a range of health-related matters and may have impacts 
on the wellbeing of patients. Yet, these are not subject to any regulations or screening procedures. Ensuring their safety, validity, and reliability should still be essential, despite them not being subject to control [1]. The Organization for Economic Co-operation and Development (OECD)-Harvard Global Health Institute expert consult suggested the development of a cross-country accreditation strategy for mobile health applications. This strategy would allow for clear labeling that provides users with guidance on the reliability of apps. Yet, it would minimize the time and resource allocations required for legislation and regulation of all apps [48].

\section{FUTURE RESEARCH}

Extensive research on the role of dietary mobile apps in chronic diseases other than obesity and diabetes would significantly contribute to the current body of knowledge[2]. This is particularly relevant for chronic conditions that require a behavioral change of multiple dietary components, such as in the case of CKD. The current dissertation provides preliminary results on a mobile application for hemodialysis patients. The results of this thesis add to the existing knowledge available in the field by contributing to the information available on the effectiveness and feasibility of dietary apps in CKD, but also by providing a unique example for the development of mobile apps within a specific cultural and professional context.

While educational dietary apps seem promising, research is also scarce in this area. Thus, further exploration of nutritional apps as supportive educational tools and therapy guidelines for patients and healthcare providers is also warranted. Our pilot study was able to show that nutrition education interventions using mobile apps in CKD may have the potential to improve patient knowledge. However, more research is needed on the role of in-app nutrition education on changing patient behavior and improving adherence.

Long-term app usage may not have the same positive effects on nutritional outcomes, as does short-term usage. A study from Norway that lasted one year found no benefits from the use of mobile apps on dietary intakes, anthropometric data, or blood parameters in patients with type 2 diabetes [49]. Accordingly, despite the promise of these interventions, more thorough evaluations that assess their effect on the long term are also needed.

App usage is another area that should be explored by future research; patient engagement is a vital component in the improvement of health outcomes. Measuring patient engagement is still not clearly defined, and multiple options have been used to measure it. Some researchers have addressed it as a psychological construct related to patient perceptions and experiences, whereas others have defined it as a behavior pertaining to the usage of an intervention [50, 
51]. Thus, engagement in mHealth interventions can be emotional, cognitive, or behavioral and can be captured through qualitative and quantitative data. Given the complexity and lack of validated and tested tools for engagement, using multiple methods may be most suitable [52]. According to Birnbaum 2015, patients do not seem to use mobile health solutions as much as they potentially could; typical retention rates of medical/fitness apps have been reported to be about 30\% [53]. An analysis of diet app users' characteristics reported that users are more likely to be females, interested in health topics, who are frequent smartphone users in general; additionally, a drive for thinness, keeping a diet and excessive exercise predicted app usage [54]. Another study looking at user characteristics in chronic disease identified that younger, more educated persons perceiving a need for physical activity and disease management were more likely to use dietary apps[55]. Characteristics and attitudes of users may be influenced by culture and could be specific within the context of chronic disease; accordingly, further research should explore more the characteristics and attitudes of users/non-users and components of apps that foster more app usage.

Given the rapid growth of technology, research must incorporate currently developing technologies; in the context of our topic, this may involve capturing dietary intakes and eating habits requiring minimal input from users. Self-reporting of dietary intakes mainly requires manual input of foods, which at times is burdensome on users. Using food photographs is less arduous; however, interpretation and translation of images, given the current technologies, might be erratic due to the estimation of portion sizes. At the moment, users can use food photographs to track intakes, but they must always use a fiducial marker (such a business card) and take multiple images from different angles[56]. Technologies that involve image recognition using artificial intelligence (AI) are increasing; they include creating datasets of the foods and combined dishes. A research team in Singapore developed an artificial intelligence (AI) based image recognition software that was trained to recognize 756 food items. However, there are still many challenges related to the addition of new food groups and the incorporation of nutrient databases linked to the software and images [57]. Given the current gap in the availability of nutrient databases in the Arab world and the development of new technologies, it may be a vanguard to launch a task force for the development of image-based databases using artificial intelligence. This would include the creation of online databases of food compositions tables coupled with corresponding datasets of food photographs. Such work could pave the ground for the growth of nutrition research and nutritional mHealth interventions in the region.

The time required to follow an iterative, formative approach is extensive; the development process of KELA.AE took more than one year to complete. This included conceptualizing, 
grounding in theories and evidence, collecting qualitative data, developing educational materials, recording, prototyping, and finally completing the first version of the app. This was then followed by pilot testing, and an additional randomized controlled trial to evaluate the app and its effectiveness would require at least another year. In the meantime, technology is continuously evolving rapidly, and the way technology is integrated into solutions is also changing. Thus, the need to create a strong collaboration between researchers and commercial app developers is crucial for effective solutions developed promptly and in line with current technologies. 


\section{REFERENCES}

1. Ferretti, A., E. Ronchi, and E. Vayena, From principles to practice: benchmarking government guidance on health apps. The Lancet Digital Health, 2019. 1(2): p. e55e57.

2. El Khoury, C.F., et al., The Effects of Dietary Mobile Apps on Nutritional Outcomes in Adults with Chronic Diseases: A Systematic Review. Journal of the Academy of Nutrition and Dietetics, 2019.

3. Yardley, L., et al., The person-based approach to intervention development: application to digital health-related behavior change interventions. Journal of medical Internet research, 2015. 17(1): p. e30.

4. Mummah, S.A., et al., IDEAS (Integrate, Design, Assess, and Share): a framework and toolkit of strategies for the development of more effective digital interventions to change health behavior. Journal of medical Internet research, 2016. 18(12): p. e317.

5. Salwen-Deremer, J.K., et al., Incorporating Health Behavior Theory into mHealth: an Examination of Weight Loss, Dietary, and Physical Activity Interventions. Journal of Technology in Behavioral Science, 2019.

6. Paschall, M.J., et al., Evaluation of an Internet-based alcohol misuse prevention course for college freshmen: Findings of a randomized multi-campus trial. American Journal of Preventive Medicine, 2011. 41(3): p. 300-308.

7. Meyer, B., et al., Effectiveness of a novel integrative online treatment for depression (Deprexis): randomized controlled trial. Journal of medical Internet research, 2009. 11(2): p. e15.

8. Whittaker, R., et al., A theory-based video messaging mobile phone intervention for smoking cessation: randomized controlled trial. Journal of medical Internet research, 2011. 13(1): p. e10.

9. Kreps, G.L. and L. Neuhauser, New directions in eHealth communication: opportunities and challenges. Patient education and counseling, 2010. 78(3): p. 329336.

10. Campbell, J. and J. Porter, Dietary mobile apps and their effect on nutritional indicators in chronic renal disease: a systematic review. Nephrology, 2015. 20(10): p. 744-751.

11. Kosa, S.D., et al., Nutritional mobile applications for CKD patients: systematic review. Kidney international reports, 2019. 4(3): p. 399-407.

12. Pollard, C.M., et al., Who uses the internet as a source of nutrition and dietary information? An Australian population perspective. Journal of medical Internet research, 2015. 17(8): p. e209. 
13. Daniel, F., et al., Patient-physician communication in the era of mobile phones and social media apps: cross-sectional observational study on Lebanese physicians' perceptions and attitudes. JMIR medical informatics, 2018. 6(2): p. e18.

14. Ammenwerth, E., From eHealth to ePatient: The Role of Patient Portals in Fostering Patient Empowerment. European Journal of Biomedical Informatics, 2018. 14(2).

15. Macdonald, G.G., et al., eHealth technologies, multimorbidity, and the office visit: qualitative interview study on the perspectives of physicians and nurses. Journal of medical Internet research, 2018. 20(1): p. e31.

16. Karavetian, M., et al., Effect of behavioral stage-based nutrition education on management of osteodystrophy among hemodialysis patients, Lebanon. Patient education and counseling, 2015. 98(9): p. 1116-1122.

17. Prochaska, J.O. and W.F. Velicer, The transtheoretical model of health behavior change. American journal of health promotion, 1997. 12(1): p. 38-48.

18. Wallace, L.S., et al., Characteristics of exercise behavior among college students: application of social cognitive theory to predicting stage of change. Preventive medicine, 2000. 31(5): p. 494-505.

19. Adams, J. and M. White, Why don't stage-based activity promotion interventions work? Health education research, 2004. 20(2): p. 237-243.

20. Marshall, S.J. and S.J. Biddle, The transtheoretical model of behavior change: a metaanalysis of applications to physical activity and exercise. Annals of behavioral medicine, 2001. 23(4): p. 229-246.

21. West, R., Time for a change: putting the Transtheoretical (Stages of Change) Model to rest. Addiction, 2005. 100(8): p. 1036-1039.

22. Brug, J., et al., The Transtheoretical Model and stages of change: a critique: observations by five commentators on the paper by Adams, J. and White, M.(2004) why don't stage-based activity promotion interventions work? Health education research, 2005. 20(2): p. 244-258.

23. Fishbein, M., A reasoned action approach to health promotion. Medical Decision Making, 2008. 28(6): p. 834-844.

24. van Doorn-van Atten, M., et al., Determinants of Behaviour Change in a MultiComponent Telemonitoring Intervention for Community-Dwelling Older Adults. Nutrients, 2018. 10(8): p. 1062.

25. Durose, C.L., et al., Knowledge of dietary restrictions and the medical consequences of noncompliance by patients on hemodialysis are not predictive of dietary compliance. Journal of the American Dietetic Association, 2004. 104(1): p. 35-41. 
26. Tentori, F., et al., Mortality risk for dialysis patients with different levels of serum calcium, phosphorus, and PTH: the Dialysis Outcomes and Practice Patterns Study (DOPPS). American Journal of Kidney Diseases, 2008. 52(3): p. 519-530.

27. Kovesdy, C.P., et al., Serum and dialysate potassium concentrations and survival in hemodialysis patients. Clinical Journal of the American Society of Nephrology, 2007. 2(5): p. 999-1007.

28. Borges, M.C.C., et al., Malnutrition Inflammation Score cut-off predicting mortality in maintenance hemodialysis patients. Clinical nutrition ESPEN, 2017. 17: p. 63-67.

29. Pignone, M.P., et al., Counseling to promote a healthy diet in adults: a summary of the evidence for the US Preventive Services Task Force. American journal of preventive medicine, 2003. 24(1): p. 75-92.

30. Almunawar, M., M. Anshari, and M.Z. Younis, Incorporating customer empowerment in mobile health. Health Policy and Technology, 2015. 4(4): p. 312-319.

31. Fakih El Khoury, C., et al., Dietary Application for the Management of Patients with Hemodialysis: A Formative Development Study. Healthcare informatics research, 2019. 25(4): p. 262-273.

32. Cashel, K. and H. Greenfield, The effects of Australian, US and UK food composition tables on estimates of food and nutrient availability in Australia. 1995.

33. Musaiger, A., Food composition tables for Kingdom of Bahrain. Food composition tables for Kingdom of Bahrain., 2011.

34. Pellet, P. and S. Shadarevian, Food composition. Tables for use in the Middle East. Food composition. Tables for use in the Middle East., 1970(Edn. 2).

35. Boyce, B., Nutrition apps: opportunities to guide patients and grow your career. Journal of the Academy of Nutrition and Dietetics, 2014. 114(1): p. 13-14.

36. Association, B.D. BDA Dietitian App. 2017 [cited 2020 10-01-2020]; Available from: https://www.bda.uk.com/professional/resources/bda_dietitian_app.

37. Rusnak, S. and P. Charney, Position of the Academy of Nutrition and Dietetics: Nutrition Informatics. Journal of the Academy of Nutrition and Dietetics, 2019. 119(8): p. $1375-1382$.

38. Hingle, M. and H. Patrick, There are thousands of apps for that: navigating mobile technology for nutrition education and behavior. Journal of nutrition education and behavior, 2016. 48(3): p. 213-218. e1.

39. Borrelli, B. and L.M. Ritterband, Special issue on eHealth and mHealth: Challenges and future directions for assessment, treatment, and dissemination. Health Psychology, 2015. 34(S): p. 1205. 
40. Chen, J., et al., The use of smartphone health apps and other mobile h ealth (mHealth) technologies in dietetic practice: a three country study. Journal of human nutrition and dietetics, 2017. 30(4): p. 439-452.

41. Lambert, K., K. Mansfield, and J. Mullan, Qualitative exploration of the experiences of renal dietitians and how they help patients with end stage kidney disease to understand the renal diet. Nutrition \& Dietetics, 2019. 76(2): p. 126-134.

42. Rai, A., et al., Understanding determinants of consumer mobile health usage intentions, assimilation, and channel preferences. Journal of medical Internet research, 2013. 15(8): p. e149.

43. Organization, W.H., Global diffusion of eHealth: making universal health coverage achievable. 2017: World Health Organization.

44. Papadopoulos, H., V.B. Sheth, and M. Wurst, Comparison of US and EU regulatory approaches to mobile health apps: use cases of myVisionTrack and USEFIL. mHealth Regulatory Environments, 2013: p. 27.

45. Martínez-Pérez, B., I. De La Torre-Díez, and M. López-Coronado, Privacy and security in mobile health apps: a review and recommendations. Journal of medical systems, 2015. 39(1): p. 181.

46. Weber, A.S., et al., Systematic thematic review of e-health research in the Gulf Cooperation Council (Arabian Gulf): Bahrain, Kuwait, Oman, Qatar, Saudi Arabia and United Arab Emirates. Journal of telemedicine and telecare, 2017. 23(4): p. 452-459.

47. Charani, E., et al., Do smartphone applications in healthcare require a governance and legal framework? It depends on the application! BMC medicine, 2014. 12(1): p. 29.

48. Ronchi, E.W., Liana \& Adler-Milstein, Julia, Mobile technology-based services for global health and wellness: Opportunities and challenges. 2017, OECD-HARVARD Global Health Institute Expert Consultation.

49. Holmen, H., et al., A mobile health intervention for self-management and lifestyle change for persons with type 2 diabetes, part 2: one-year results from the Norwegian randomized controlled trial RENEWING HEALTH. JMIR mHealth and uHealth, 2014. 2(4): p. e57.

50. Perski, O., et al., Conceptualising engagement with digital behaviour change interventions: a systematic review using principles from critical interpretive synthesis. Translational behavioral medicine, 2017. 7(2): p. 254-267.

51. Walton, H., et al., Measures of fidelity of delivery of, and engagement with, complex, face-to-face health behaviour change interventions: A systematic review of measure quality. British journal of health psychology, 2017. 22(4): p. 872-903. 
52. Short, C.E., et al., Measuring engagement in eHealth and mHealth behavior change interventions: viewpoint of methodologies. Journal of medical Internet research, 2018. 20(11): p. e292.

53. Birnbaum, F., et al., Patient engagement and the design of digital health. Academic emergency medicine: official journal of the Society for Academic Emergency Medicine, 2015. 22(6): p. 754.

54. Elavsky, S., D. Smahel, and H. Machackova, Who are mobile app users from healthy lifestyle websites? Analysis of patterns of app use and user characteristics. Translational Behavioral Medicine, 2017. 7(4): p. 891-901.

55. Sun, L., et al., Facilitators and barriers to using physical activity smartphone apps among Chinese patients with chronic diseases. BMC medical informatics and decision making, 2017. 17(1): p. 44.

56. Pendergast, F.J., et al., Evaluation of a smartphone food diary application using objectively measured energy expenditure. International Journal of Behavioral Nutrition and Physical Activity, 2017. 14(1): p. 30.

57. Sahoo, D., et al., FoodAI: Food Image Recognition via Deep Learning for Smart Food Logging. 2019. 

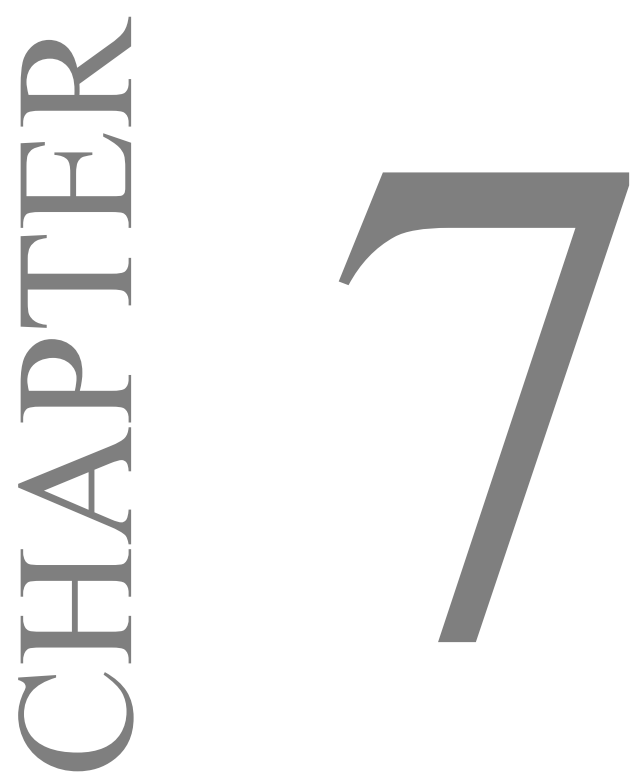

SUMMARY 
In most chronic diseases, dietary and lifestyle changes are integral components of disease management. In Chronic Kidney Disease (CKD), treatment requires nutrition interventions that aim at controlling and minimizing adverse outcomes. Dietary interventions necessitate a high level of involvement by patients, because changing eating habits is a challenging task. This also influences the interactions between dietitians and patients. Patients may feel a loss of autonomy due to the restrictions imposed by the diet and dietitians providing positive and sensitive information may encourage autonomy.

The use of mobile devices in healthcare has increased exponentially over the past decade. Mobile apps set off new possibilities across many practice areas, including dietetics. Mobile health (mHealth) has the potential to deliver nutritional care to individuals and to support practitioners by providing tools that may contribute to dietetic care in health and disease.

This dissertation aims to provide insight into the available evidence on the effectiveness of dietary applications in chronic disease and to explore the feasibility of developing and pilot testing an mHealth intervention using an Arabic dietary app for hemodialysis patients (KELA.AE).

In Chapter 2, we conducted a systematic review of the available literature along with a randomeffect meta-analysis of interventions using dietary applications. Data were extracted and clustered by studies using mobile apps as a sole intervention, comprehensive interventions comparing mobile apps to other tools, and counseling interventions supported by a mobile app. Most of the articles identified targeted obesity and diabetes.

The systematic review and meta-analysis that we performed confirmed that dietary mobile apps may have positive effects on nutritional outcomes. Most of these were self-monitoring apps used in the short-term, and outcomes were particularly related to weight management. Pooled estimates resulted from the random-effect meta-analysis showed significant improvements in weight, waist circumference, and energy intake of participants using dietary apps. Mobile apps that were used a sole intervention were found to be effective self-monitoring tools. Nevertheless, when compared to other tools (paper-based diaries, calorie reference books, or a self-monitoring website), we were unable to identify a clear superiority for mobile apps. Theory-based dietary apps used in a counseling intervention were found to be effective and thus may provide additional benefits. While educational dietary apps seem promising, not enough research is available in this field; only two studies identified apps that were used as educational tools. 
In Chapter 3, we describe the development of the application. Kidney Education for Lifestyle Application (KELA.AE app) is a self-monitoring and educational app for Arabic speaking hemodialysis patients. The name KELA in Arabic signifies Kidney and.AE was selected as the suffix in the app name to relate the app to the Internet domain name of the United Arab Emirates (UAE). KELA.AE app was developed based on the Integrate, Design, Assess, and Share (IDEAS) Framework [1].

Qualitative, semi-structured interviews with hemodialysis patients, dietitians, and nephrologists were performed. The patients interviewed during the development reported using the Internet usage as a source for nutrition information. Patients also expressed frustration with the diet experience in general, which they considered challenging. The results of the interviews with patients stressed a need for education rather than self-monitoring.

Dietitians and nephrologists considered the app a useful tool for patients. However, some expressed concern regarding app misuse and replacement of healthcare practitioners. They expressed fear that patients would exclusively rely on the app instead of the dietitian or physician.

KELA.AE was developed in a stepwise formative person-centered approach. Existing evidence and theories were incorporated, and the content was culturally adapted. Overall, KELA.AE app was designed to contain a total of 24 podcasts, three animated videos, and 161 notifications. All educational materials were developed based on previously validated print Arabic dietary educational materials[2] amended to accommodate for the needs of the participants' culture (Emirate dialect, local traditional recipes...) and the educational modalities included in the app (podcasts, videos, and notifications). All educational materials developed are based on the Transtheoretical Model for behavior change[3]; however, the narration incorporates additional constructs from the Reasoned Action Approach[4].

In Chapters 4 and 5, we conducted a pilot study to assess the feasibility of an mHealth intervention using the KELA.AE app along with a face-to-face intervention. We also investigated the potential efficacy that KELA.AE usage may have on nutritional outcomes. We presented the data in two manuscripts, one describing the results of the feasibility of an mHealth intervention on dietary intakes and diet quality, the other describing phosphorus management in hemodialysis exploring the role of the educational features of the app.

In Chapter 4, we report the findings of the pilot on the feasibility and pilot testing the effects of the KELA.AE app on dietary intakes, anthropometric measurements, laboratory parameters, and adherence to current dietary guidelines. Our findings show that KELA.AE app is feasible to be used during mHealth interventions as an educational and self-monitoring tool. The short- 
term usage may have a potential impact on improving energy and protein intakes among hemodialysis patients. However, no changes were observed in intakes of minerals; fat intakes were elevated at baseline and remained above recommendations after the intervention. No changes were observed in the anthropometric parameters and serum laboratory parameters for potassium and phosphorus. Serum Fe increased, which may be explained by the increase in the dietary protein of high biological value. Overall, we found the use KELA.AE to be a feasible tool used with a face-to-face intervention to address dietary intakes of hemodialysis patients. A larger randomized controlled trail is necessary to provide information on the efficacy of the app.

In Chapter 5, we reported the feasibility of using the KELA.AE in-app education on the phosphorus management of hemodialysis patients. We measured outcomes on patient knowledge, self-reported non-adherence, phosphorus intakes, and blood parameters.

The main findings of this pilot study show that in-app nutrition education is feasible as a supportive tool for dietitians and patients. Our study found that short-term app usage may improve patient knowledge; nevertheless, we found no improvement in dietary adherence. Surprisingly non-adherence to the phosphorus content of the diet increased after the intervention. This may be explained by the self-perception of adherence influenced by patient knowledge. Patients might have become more aware of the phosphorus content of food, and therefore, their self-perception of non-adherence changed accordingly.

Phosphorus intakes did not change after the pilot intervention. An increase in dietary protein, however, was observed, as reported in chapter 4. Guidelines on protein and phosphorus intakes in hemodialysis are somewhat contradictory, and thus achieving both is challenging, and patients often do not consume enough proteins to restrict phosphorus. Nevertheless, the increase in protein intakes did not negatively impact phosphorus intakes in this pilot study. Furthermore, the phosphorus to protein ratio post-intervention was nearer to the recommended ratio of $10-12 \mathrm{mg} / \mathrm{g}$ protein [5], possibly meaning that patients increased their protein intakes while choosing foods that were lower in phosphorus.

The findings of this thesis present several implications for the practice setting and research. First, it provides evidence on the effectiveness of dietary apps in chronic diseases. Second, it provides insights for developers and dietetic practitioners on the formative development steps to be followed based on an in-person, evidence, and theory-based approach. We also provide the feasibility and potential efficacy based on the results of a pilot study evaluating an mHealth intervention using the KELA.AE app. Finally, this thesis generates directions for future research in the area of mHealth applied to dietetic practice in the context of chronic disease. 


\section{REFERENCES}

1. Mummah, S.A., et al., IDEAS (Integrate, Design, Assess, and Share): a framework and toolkit of strategies for the development of more effective digital interventions to change health behavior. Journal of medical Internet research, 2016. 18(12): p. e317.

2. Karavetian, M., et al., Effect of behavioral stage-based nutrition education on management of osteodystrophy among hemodialysis patients, Lebanon. Patient education and counseling, 2015. 98(9): p. 1116-1122.

3. Prochaska, J.O. and W.F. Velicer, The transtheoretical model of health behavior change. American journal of health promotion, 1997. 12(1): p. 38-48.

4. Fishbein, M., A reasoned action approach to health promotion. Medical Decision Making, 2008. 28(6): p. 834-844.

5. Kopple, J.D., National kidney foundation K/DOQI clinical practice guidelines for nutrition in chronic renal failure. American journal of kidney diseases, 2001. 37(1): p. S66-S70. 
VALORIZATION 


\section{VALORIZATION}

The use of eHealth has become a reality that practitioners can benefit from; technologies are continually evolving, and they have become an integral part of societies.

The present thesis explored the effectiveness of dietary apps on nutritional outcomes in chronic diseases as well as the development and pilot evaluation of an Arabic dietary self-monitoring and educational app for hemodialysis patients. The app developed (KELA.AE) is to our knowledge the first of its kind in the Arab region. The valorization of the thesis may be directed to clinicians, app developers, policymakers, and researchers to provide optimal nutrition care to patients while incorporating the use of mobile technologies. This thesis could also be considered a roadmap for researchers and app developers regarding person-centered, evidence, and theory-based approaches that could be used for the development of dietary apps for the management of chronic diseases.

\section{COLLABORATION BETWEEN DEVELOPERS AND STAKEHOLDERS}

The involvement of all stakeholders is essential for the development of tools that succeed in patient care. Historically, patients have rarely been actively involved in the development of healthcare tools. The process involved healthcare practitioners, developers, and scientists rather than patients themselves. However, patients are interested in personalized, interactive tools to self-manage their conditions; they want customized, targeted, tailored information to satisfy their needs.[1]. Patient engagement has been linked to better health outcomes, yet there have been reports of low usage because existing tools generally fail to cater to patients' needs[2]. Technologies will only succeed if patients are ready, motivated, willing, capable of using them and find them easy to use

Additionally, commercial dietary mobile applications available through app stores are not always grounded in theories and evidence, and they often lack accuracy in the information provided and do not go through extensive evaluations [3-5]. Whereas, applications based on formative research are more likely to be grounded in theories and therapeutic evidence, yet they may not be well disseminated, nor do they benefit from updates from the rapidly evolving technologies[6]. Thus, there is a need to disseminate interventions that have undergone trials showing effectiveness and to provide them as open access to the public. Dissemination should be made for research, particularly in open access journals, to share results. App dissemination, on the other hand, should also be performed and potentially be in collaboration with the industry. 
If dietitians, and healthcare practitioners, in general, do not take the lead by contributing to mhealth developments, the rapid technological advances may be disruptive or possibly even cause harm to patient management [7, 8]. The latter may allow for the dissemination and usage of mHealth solutions that are not grounded in theories and therapeutic evidence, which in turn may have a negative impact on health outcomes.

We propose a close collaboration between app developers, dietitians, and patients as means to approach such challenges with an overarching tactic that allows patients to benefit from the theories and evidence produced by the scientific community but also from the technological advances and entrepreneurial engines made by the private sector. We perceive that for this idea to take shape, dietitians and other healthcare practitioners should take the lead by approaching this continuously evolving sector and therefore contribute to the development, dissemination, and evaluation of dietary applications or other future technologies that may arise. Dietitians and healthcare practitioners, in general, should also act as a liaison to ensure patient involvement in the development process so that solutions are adequately catered to patient needs. To accomplish the latter, dietitians must obtain and continuously update their knowledge and skills in the area of nutrition informatics.

The Commission on Dietetic Registration of the Academy of Nutrition and Dietetics in the United States has taken the lead by incorporating informatics related competencies that dietitians may choose to fulfill as part of the continuing education required to maintain their credentialing. The Commission on Dietetic Registration, however, has not made these learning outcomes mandatory; we suggest that the informatics sphere is made compulsory for the renewal of dietitians' credentials[9]. We also suggest that countries that require continuing education for the maintenance of credentialing should incorporate a minimum number of continuing education units in the area of nutrition informatics. This would provide dietitians with knowledge and skills to be more involved in the incorporation of advancing technologies into practice.

\section{INTEGRATION OF MHEALTH COMPONENTS INTO THE NUTRITION CARE PROCESS (NCP)}

The nutrition care process (NCP) is a framework proposed by the Academy of Nutrition and Dietetics in the United States and adopted by many countries across the world that provides a stepwise approach for the delivery of quality nutrition care. The nutrition care process (NCP) model also includes a graphical illustration that includes environmental factors that may impact the practice in dietetics. The current model lists the patient at the center surrounded by healthcare, social, economic systems, and practice settings as environmental factors that influence nutrition care practices [10]. 
We propose that during the next update of the NCP, an additional factor entitled "technological developments" is considered for addition to the outer ring of environmental factors in the model to keep the model in line with modern developments. The process may involve an update of the evidence on the role of technological developments on the practice along with a Delphi study aimed at defining the role of technological progress on dietetic practice as well as selecting where exactly they fit in the model.

Additionally, the steps included in the NCP comprise nutrition assessment, diagnosis, intervention, monitoring, and evaluation. Standardized terminology is proposed by the (NCP) pertinent to each step. The current domains for each step lack explicit incorporation for eHealth tools applied to assessment, diagnosis, interventions, or monitoring and evaluation. We also propose that the terminology should be inclusive of eHealth across all domains of the NCP. Currently, nutrition standardized terminology is incorporated into patients' electronic health records (EHR). However, the terminology itself still lacks to reflect interventions using tools such as mobile apps, web-based platforms, teleconsultations, or other components of eHealth that may contribute to the steps of the nutrition care process.

\section{APP GOVERNANCE CONSIDERATIONS}

Intensive nutrition interventions are clinically and cost-effective in the management of hemodialysis patients, and particularly in the management of hyperphosphatemia [11]. However, this requires a dietitian to patient ratio along with enough time dedicated to each patient [12]. This is a challenge that most healthcare systems face, especially in the dietetics field, where clinical nutrition staffing benchmarks are not yet well established [13]. Dietary apps can offer support to dietitians and patients/clients equally as they may act as educational, self-monitoring, or communication tools. The aim would be to enhance the dietitian and patient relationship by providing tools that render the communication more efficient.

New apps are being continuously developed and made accessible to the public; these apps often contain target guidelines, educational materials, and require the input of personal health information. Given their potential contribution to patients' treatment plans, special attention should be given to their content and to the management of data that users input into the app.

Different countries have regulations mandating data protection and data processing; such laws also cover matters related to users consenting to share their personal data $[14,15]$. However, app content, reliability, and correctness in line with current practice guidelines are not regulated. Placing rigid regulations may hinder technological development, yet not including any overarching view of the scope of these apps may put users at risk. 
mHealth apps should be assessed and validated before being made available to the public, and thus app evaluation should be more thoroughly explored. In France, the Haute Autorite de Sante developed good practice guidelines for manufactures and evaluators for apps that are not subject to regulatory approvals (such as apps with medical purposes)[16]. Tools evaluating apps such as the MARS (Mobile App Rating Scale) have also been developed, validated, and extensively used in the literature for app evaluation [17]. At the international level, however, there is no consensual approach to the assessment of mobile apps that are not classified as medical devices.

An international task force guiding the evaluation and dissemination of apps could be a future direction for policymakers and researchers. The task force would create a universal app evaluation tool that is adapted from validated tools. It may include international healthcare practitioners, app developers, patients, but also stakeholders from commercial apps. It would also aim at creating a voluntary "label" similar to a food label. The label would be available to app stores, and it should include easy to understand criteria with a rating of the app. The task force would also create guidelines for the development of an app label that includes reliability of content (compliance to evidence practice guidelines), data privacy and management, developer qualifications, among other criteria. Such labels would serve as a guiding tool for dietitians, healthcare practitioners, and patients equally.

\section{References}

1. Kaplan, B. and P.F. Brennan, Consumer informatics supporting patients as coproducers of quality. Journal of the American Medical Informatics Association, 2001. 8(4): p. 309-316.

2. Birnbaum, F., et al., Patient engagement and the design of digital health. Academic emergency medicine: official journal of the Society for Academic Emergency Medicine, 2015. 22(6): p. 754.

3. Azar, K.M., et al., Mobile applications for weight management: theory-based content analysis. American journal of preventive medicine, 2013. 45(5): p. 583-589.

4. Pagoto, S., et al., Evidence-based strategies in weight-loss mobile apps. American journal of preventive medicine, 2013. 45(5): p. 576-582.

5. Chen, J., J.E. Cade, and M. Allman-Farinelli, The most popular smartphone apps for weight loss: a quality assessment. JMIR mHealth and uHealth, 2015. 3(4): p. e104. 
6. Baker, T.B., D.H. Gustafson, and D. Shah, How can research keep up with eHealth? Ten strategies for increasing the timeliness and usefulness of eHealth research. Journal of medical Internet research, 2014. 16(2): p. e36.

7. Robinson, T.N., et al., An evidence-based approach to interactive health communication: A challenge to medicine in the information age. Jama, 1998. 280(14): p. 1264-1269.

8. Rusnak, S. and P. Charney, Position of the Academy of Nutrition and Dietetics: Nutrition Informatics. Journal of the Academy of Nutrition and Dietetics, 2019. 119(8): p. $1375-1382$.

9. Worsfold, L., B.L. Grant, and G.C. Barnhill, The essential practice competencies for the Commission on Dietetic Registration's credentialed nutrition and dietetics practitioners. Journal of the Academy of Nutrition and Dietetics, 2015. 115(6): p. 978984.

10. Lacey, K. and E. Pritchett, Nutrition care process and model: ADA adopts road map to quality care and outcomes management. Journal of the Academy of Nutrition and Dietetics, 2003. 103(8): p. 1061-1072.

11. Rizk, R., et al., Cost-effectiveness of dedicated dietitians for hyperphosphatemia management among hemodialysis patients in Lebanon: results from the Nutrition Education for Management of Osteodystrophy trial. Journal of medical economics, 2017. 20(10): p. 1024-1038.

12. Byham-Gray, L. and K. Wiesen, A clinical guide to nutrition care in kidney disease. 2004: American Dietetic Associati.

13. Phillips, W., Clinical nutrition staffing benchmarks for acute care hospitals. Journal of the Academy of Nutrition and Dietetics, 2015. 115(7): p. 1054-1056.

14. Crutzen, R., G.-J. Ygram Peters, and C. Mondschein, Why and how we should care about the General Data Protection Regulation. Psychology \& health, 2019. 34(11): p. $1347-1357$.

15. Martínez-Pérez, B., I. De La Torre-Díez, and M. López-Coronado, Privacy and security in mobile health apps: a review and recommendations. Journal of medical systems, 2015. 39(1): p. 181.

16. de Santé, H.A., Good practice guidelines on health apps and smart devices (mobile health or mhealth). 2016, URL: https://www. has-sante. fr/portail/upload/docs/application/pdf/2017-03 ....

17. Stoyanov, S.R., et al., Mobile app rating scale: a new tool for assessing the quality of health mobile apps. JMIR mHealth and uHealth, 2015. 3(1): p. e27. 
LIST OF PUBLICATIONS OF THE THESIS 
Fakih El Khoury, C., Karavetian, M., Halfens, R. J., Crutzen, R., Khoja, L., \& Schols, J. M. (2019). The effects of dietary mobile apps on nutritional outcomes in adults with chronic diseases: a systematic review. Journal of the Academy of Nutrition and Dietetics.

Fakih El Khoury, C., Karavetian, M., Halfens, R. J., Crutzen, R., El Chaar, D., \& Schols, J. M. (2019). Dietary Application for the Management of Patients with Hemodialysis: A Formative Development Study. Healthcare informatics research, 25(4), 262-273.

Fakih El Khoury C., Crutzen R, Schols JM, Halfens RJ, Karavetian M (2020). Adequate management of Phosphorus in hemodialysis using a dietary smartphone app: A pilot study. JMIR Preprints. 17/01/2020:17858 (submitted)

Fakih El Khoury C., Crutzen R, Schols JM, Halfens RJ, Karavetian M (2020). Adequate management of Phosphorus in hemodialysis using a dietary smartphone app: A pilot study. JMIR Preprints. 17/01/2020:1785 (submitted) 
ACKNOWLEDGMENTS 
The journey of obtaining a Ph.D. has been enriching, challenging, and rewarding in every way. It has taught me to leap into change and transform obstacles into learning opportunities.

I would like to thank all the people whose support was a milestone in the completion of this journey. Foremost I am grateful to Maastricht University and my supervisors, Prof. Dr. Jos M.G.A. Schols, Prof. Dr. Rik Crutzen, Dr. Ruud J.G. Halfens, and Dr. Mirey Karavetian. I was lucky to have a team always providing support, motivation, and immense knowledge. I could not have imagined having better advisors and mentors.

Prof. Dr. Jos M.G.A. Schols, thank you for always providing me with encouraging and super-fast feedback; Prof. Dr. Rik Crutzen for challenging me to explore new knowledge, Dr. Ruud Halfens for providing me with your guidance and expertise during every Ph.D. group meeting. Dr. Mirey Karavetian, your presence in my life goes above and beyond this Ph.D., you have opened every closed door, supported me at the professional and personal level, and provided me with numerous opportunities. As always, you never cease to amaze me with your generosity, and kindness.

I would like to thank the Ph.D. group supervisors Prof. Dr. Christa Lohrmann and Prof. Dr. Sabine Hahn, for their valuable inputs. To my fellow Ph.D. students on the program, I enjoyed every discussion, idea, thought, and support that each one of you has provided me. I will miss every trip to Graz, Bern, and Maastricht. I especially would like to thank Dr. Friederike Thilo for the inspiring ideas and advice that I will always seek, and (Dr. to be) Simone Paulis, I will miss our chats, walks, and the lovely times we shared.

I would like to thank Zayed University and Al Qassimi Hospital for providing me with the opportunity to perform my research. A special thank you to all the hemodialysis patients, dietitians, and physicians that have accepted to participate in the studies. A big thank you to the dietetic students, faculty members, and departments of Zayed University who have contributed to the development of the KELA.AE app or to the research. I would also like to thank the team from S4M for the development of the app.

To my wonderful family, Johnny and Siena, I thank you for your patience, support, encouragement, and immense love during this phase and always. To my parents (Mounir and Salam) for being my backbone and my siblings (Nizar and Lara) for being my safety net. I love you all immensely, and I am grateful to have you in my life. I am also thankful to all my friends and colleagues who have encouraged me and who stood by me during the past four years. I would like to give a special thank you to Sandy who designed the cover page; she always contributes with her "touch" to each one of my milestones (personal and professional). 
ABOUT THE AUTHOR 


\section{AbOUT THE AUTHOR}

Cosette Fakih was born on April 4th, 1981 in Lebanon. She obtained her Bachelor of Sciences in Nutrition and Dietetics in 2004 and her master of sciences in Human Nutrition in 2009 from the American University of Beirut, Lebanon.

Cosette Fakih is a licensed dietitian by the Ministry of Public Health in Lebanon; she worked as a Clinical Dietitian, a dietetic internship coordinator and acting head of dietary division at a governmental university hospital in Lebanon. She was among the starting team of a Nutrition Center in Qatar where she was the Head Dietitian. She has worked as an instructor at several universities including the Lebanese University, the American University of Beirut, and Qatar University. She has worked on accreditation of dietetic programs as a consultant. She is currently an instructor and clinical preceptor at the Lebanese American University. 


\section{PEER-REVIEWED ARTICLES}

Fakih El Khoury, C., Karavetian, M., Halfens, R. J., Crutzen, R., Khoja, L., \& Schols, J. M. (2019). The effects of dietary mobile apps on nutritional outcomes in adults with chronic diseases: a systematic review. Journal of the Academy of Nutrition and Dietetics.

Fakih El Khoury, C., Karavetian, M., Halfens, R. J., Crutzen, R., El Chaar, D., \& Schols, J. M. (2019). Dietary Application for the Management of Patients with Hemodialysis: A Formative Development Study. Healthcare informatics research, 25(4), 262-273.

Fakih El Khoury C., Crutzen R, Schols JM, Halfens RJ, Karavetian M (2020). Adequate management of Phosphorus in hemodialysis using a dietary smartphone app: A pilot study. JMIR Preprints. 17/01/2020:17858 (submitted)

Fakih El Khoury C., Crutzen R, Schols JM, Halfens RJ, Karavetian M (2020). Adequate management of Phosphorus in hemodialysis using a dietary smartphone app: A pilot study. JMIR Preprints. 17/01/2020:1785 (submitted)

Salhab, N., Karavetian, M., Kooman, J., Fiaccadori, E., \& Fakih El Khoury, C. (2019). Effects of intradialytic aerobic exercise on hemodialysis patients: a systematic review and meta-analysis. Journal of nephrology, 1-18.

Bawadi, H., Katkhouda, R., Al-Haifi, A., Tayyem, R., Fakih El Khoury, C., \& Jamal, Z. (2016). Energy balance and macronutrient distribution in relation to C-reactive protein and HbA1c levels among patients with type 2 diabetes. Food \& nutrition research, 60(1), 29904.

Bawadi, H. A., Bunks, A. D., Tayyem, R. F., \& Fakih El Khoury, C. (2016). Meals served to hypertensive and cardiac inpatients in Jordan: comparison with WHO and NIH dietary guidelines. EMHJ-Eastern Mediterranean Health Journal, 22(1), 28-33. 